


\begin{abstract}
SPIRITUAL COPING AS A MEDIATOR OF DISTRESS AND POSTTRAUMATIC GROWTH AMONG ADULT FEMALE SURVIVORS OF SEXUAL ABUSE BY RELIGIOUS LEADERS
\end{abstract}

by

Angela R. St. Hillaire

Chair: Elvin Gabriel 


\section{ABSTRACT OF GRADUATE STUDENT RESEARCH}

Dissertation

Andrews University

School of Education

\section{Title: SPIRITUAL COPING AS A MEDIATOR OF DISTRESS AND POSTTRAUMATIC GROWTH AMONG ADULT FEMALE SURVIVORS OF SEXUAL ABUSE BY RELIGIOUS LEADERS}

Name of researcher: Angela St. Hillaire

Name and degree of faculty chair: Elvin Gabriel, EdD

Date Completed: July 2020

\section{Problem}

Posttraumatic growth has been defined as the positive psychological and behavioral changes that come about in the aftermath of a struggle with traumatic life events. The literature notes the existence of posttraumatic growth among survivors of intimate partner violence, childhood and/or adult sexual abuse, bereavement, terrorism, and other events. This study explored posttraumatic growth in a sample of female survivors of sexual abuse by religious leaders by examining how distress, spiritual coping, and posttraumatic growth were related in this population. This study examined the mediatory role of spiritual coping between distress and posttraumatic growth. 
Method

Surveys that measured spiritual coping, distress, posttraumatic growth, and the context of abuse were completed by 113 participants. The following were inclusion criteria for the sample: female identification, age 18 or older, and an experience of sexual abuse by religious leaders. The resulting data was analyzed using descriptive statistics, Path Analysis, and Structural Equation Modeling.

\section{Results}

Path Analysis indicated that positive spiritual coping, but not negative spiritual coping, was a predictor of PTG. High distress was also found to directly predict posttraumatic growth. However, distress and spiritual coping were not related to one another. Consequently, spiritual coping did not mediate the relationship between distress and posttraumatic growth.

\section{Conclusions}

While this study did not find that spiritual coping played a mediatory role in the relationship between distress and posttraumatic growth, it provided evidence of a direct correlation between high distress and posttraumatic growth. This finding supports the literature suggesting a positive link between these two constructs. Additionally, the fact that negative spiritual coping was unrelated to posttraumatic growth while positive spiritual coping directly predicted posttraumatic growth lends support to the

distinctiveness of these coping styles and suggests that certain styles of spiritual coping are more likely to facilitate growth than others. Lastly, the link between positive spiritual coping and posttraumatic growth suggests that positive spiritual coping can be a beneficial resource for female survivors of sexual abuse by religious leaders. 
Andrews University

School of Education

SPIRITUAL COPING AS A MEDIATOR OF DISTRESS AND POSTTRAUMATIC GROWTH AMONG ADULT FEMALE SURVIVORS OF SEXUAL ABUSE BY RELIGIOUS LEADERS

\author{
A Dissertation \\ Presented in Partial Fulfillment \\ of the Requirements for the Degree \\ Doctor of Philosophy
}

by

Angela R. St. Hillaire

July 2020 
C Copyright by Angela R. St. Hillaire 2020

All Rights Reserved 


\title{
SPIRITUAL COPING AS A MEDIATOR OF DISTRESS AND POSTTRAUMATIC GROWTH AMONG ADULT FEMALE SURVIVORS OF SEXUAL ABUSE BY RELIGIOUS LEADERS
}

\author{
A dissertation \\ presented in partial fulfillment \\ of the requirements for the degree \\ Doctor of Philosophy
}

by

Angela R. St. Hillaire

APPROVAL BY THE COMMITTEE:

Chair: Elvin Gabriel

Member: Nancy Carbonell

Member: Jimmy Kijai

External: Lionel Matthews
Dean, College of Education and

International Services

Alayne Thorpe

Date approved 


\section{TABLE OF CONTENTS}

LIST OF FIGURES

LIST OF TABLES .......................................................................... viii

LIST OF ABBREVIATIONS …..............................................................

ACKNOWLEDGEMENTS ...........................................................................

Chapter

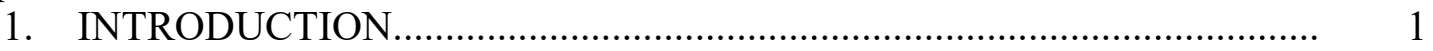

Introduction ....................................................................... 1

Rationale for the Study ................................................................ 4

Statement of the Problem.................................................................... 4

Purpose of the Study .................................................................. 6

Conceptual Framework ................................................................. 7

Theoretical Model of PTG in Survivors of SARL........................ 8

Research Questions ............................................................... 13

Hypothesis................................................................................ 13

Significance of the Study ........................................................... 15

Definition of Terms ................................................................ 17

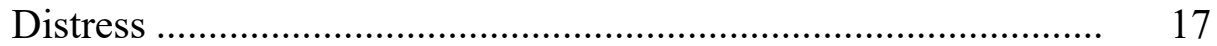

Event-Related Rumination....................................................... 17

Meaning Making ....................................................................... 17

Posttraumatic Growth ............................................................ 18

Sexual Abuse by Religious Leaders ......................................... 18

Spiritual Coping ................................................................ 18

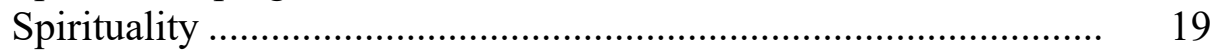

Delimitations ............................................................................ 19

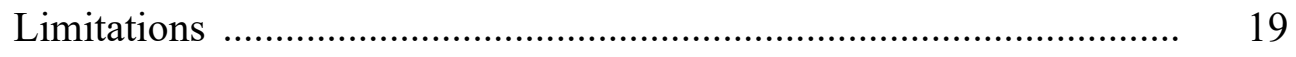

Organization of the Study .......................................................... 20

2. LITERATURE REVIEW .............................................................. 21

Introduction................................................................................ 21

Posttraumatic Growth: An Overview.............................................. 22

Predictors of Posttraumatic Growth.......................................... 28

Posttraumatic Growth: Cognitive Processing and 
Event-Related Rumination .................................................... 31

Posttraumatic Growth: Narratives and Meaning Making ............... 34

Posttraumatic Growth and Religious/Spiritual Coping ................. 38

Posttraumatic Growth Among Female Survivors of Sexual Abuse....... $\quad 42$

Posttraumatic Growth and Psychological Distress .............................. 44

Psychological Distress: An Overview ................................................... 51

Psychological Distress and Spiritual/Religious Coping ……………... 55

Psychological Distress and Spirituality ................................................ 59

Psychological Distress Among Female Survivors of Sexual Abuse ..... 61

Psychological Distress Among Female Survivors of

Sexual Abuse by Religious Leaders

Coping Mechanisms Used by Female Survivors of

Sexual Abuse by Religious Leaders ............................................... 63

Spiritual Coping: An Overview .............................................................. 69

Spiritual Coping and Posttraumatic Growth ........................................ 74

The Significance of Spirituality in Survivors of

Sexual Abuse by Religious Leaders

Spiritual Coping, Psychological Distress, and Posttraumatic

Growth Among Female Survivors of Sexual Abuse

Spiritual Coping, Psychological Distress, and Posttraumatic Growth

Among Female Survivors of Sexual Abuse

by Religious Leaders

Summary and Analysis of the Literature Review ................................. 84

3. METHODOLOGY

Introduction

Research Questions and Hypothesis ................................................. 87

Research Design .......................................................................... 88

Participants ........................................................................ 89

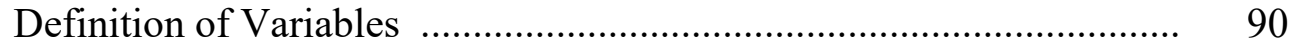

Distress ..................................................................... 90

Spiritual Coping ........................................................................ 91

Posttraumatic Growth ................................................................ 91

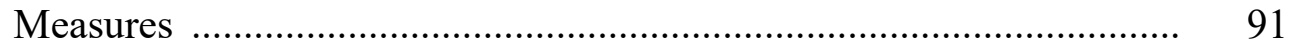

Demographic Data Sheet ........................................................... 92

Context of Abuse Questionnaire.................................................. 92

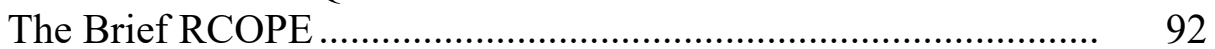

Posttraumatic Growth Inventory ................................................... 94

Emotion Thermometers ............................................................ 96

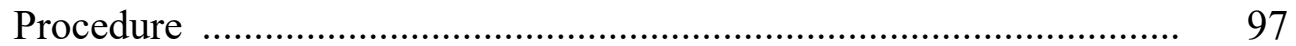

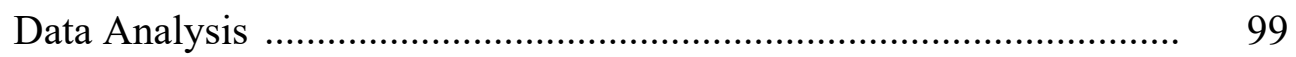

Summary ........................................................................... 100

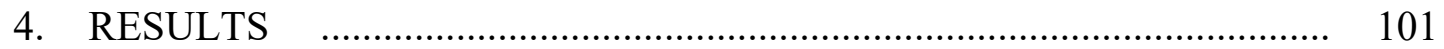




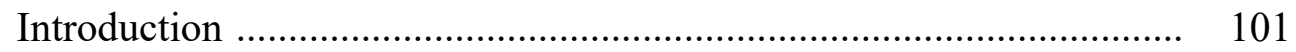

Description of Sample ………………………….......................... 101

Reliability Estimates ....................................................................... 105

Posttraumatic Growth Inventory................................................. 105

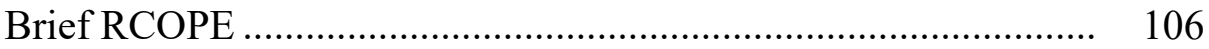

Levels of Distress, Spiritual Coping, and Posttraumatic Growth ........ 107

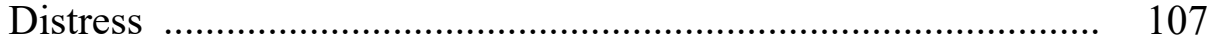

Spiritual Coping ……………………..................................... 108

Posttraumatic Growth ............................................................ 110

Results by Research Question ........................................................... 114

Question 1: Context of Abuse ...................................................... 114

Question 2: Effects of Spiritual Coping on Posttraumatic Growth 118

Question 3: Effects of Distress on Posttraumatic Growth ............. 121

Question 4: Spiritual Coping as a Mediator between

Distress and Posttraumatic Growth ...................................... 125

Summary of Findings ............................................................. 129

5. SUMMARY, DISCUSSION, AND RECOMMENDATIONS .................... 131

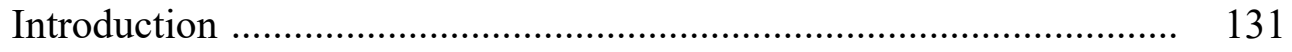

Research Problem …………………………….......................... 131

Purpose of the Study ................................................................... 132

Significance of the Study ................................................................... 133

Summary of the Literature ............................................................. 134

Methodology ............................................................................. 137

Demographic Characteristics of Respondents .................................. 138

Summary and Discussion of Major Findings ...................................... 139

Research Question 1 .............................................................. 139

Research Questions 2 .......................................................... 142

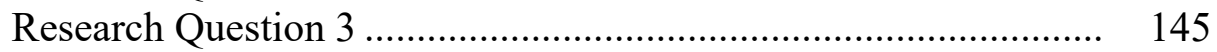

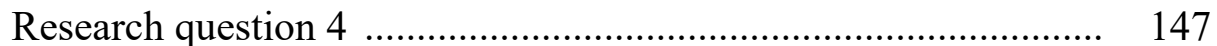

Limitations of the Study ................................................................. $\quad 150$

Recommendations for Practice ........................................................ 151

Recommendations for Future Research ............................................ 152

Appendix

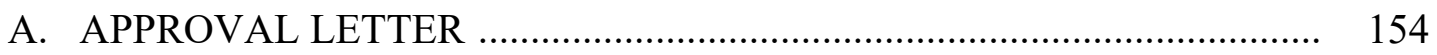

B. INFORMED CONSENT _.............................................................. 156

C. DEMOGRAPHIC DATA SHEET …………………........................... 158

D. CONTEXT OF ABUSE QUESTIONNAIRE ............................................ 161

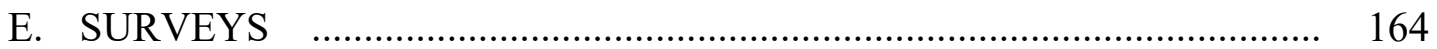


REFERENCE LIST

VITA

204 


\section{LIST OF FIGURES}

1. A Model of Posttraumatic Growth Adapted from Calhoun and Tedeschi (2006) 14

2. Hypothesized Relationship Between Distress, Spiritual Coping and Posttraumatic Growth......................................................................... 15

3. Path Diagram of the Hypothesized Model Between ..........................................

Spiritual Coping and Growth .................................................................... 118

4. Re-Specified Path Model Relating Positive Spiritual Coping and Posttraumatic Growth....................................................................... 121

5. Hypothesized Path Model Relating Distress and Posttraumatic Growth ............. 122

6. Re-Specified Path Model Relating Distress and Posttraumatic Growth .............. 124

7. Hypothesized Path Model Relating Distress, Positive Spiritual Coping, and Posttraumatic Growth

8. Re-Specified Path Model Relating Distress, Positive Spiritual Coping, and Posttraumatic Growth. 


\section{LIST OF TABLES}

1. Participants' Demographic Statistics.......................................................... 103

2. Reliability Coefficients for Scales............................................................. 107

3. Subscale Means, Medians, Standard Deviations and Skewness ....................... 108

4. RCOPE PRC Scale Mean, Standard Deviation, and Percent by Item................. 109

5. RCOPE NRC Subscale Mean, Standard Deviation, and Percent by Item .......... 110

6. PTGI Relating to Others Subscale Mean, Standard Deviation, and Percent by Item

7. PTGI New Possibilities Subscale Mean, Standard Deviation, and Percent by Item.

8. PTGI Personal Strength Subscale Mean, Standard Deviation, and Percent by Item

9. PTGI Spiritual Change Subscale Mean, Standard Deviation, and Percent by Item

10. PTGI Appreciation of Life Subscale Mean, Standard Deviation, and Percent by Item

11. Context of Abuse Subscale.

12. Path Coefficients Between RCOPE and Posttraumatic Growth (Re-Specified Model)

13. Model Summaries for RCOPE and Posttraumatic Growth

14. Path Coefficients Between Distress and Posttraumatic Growth (Re-Specified Model).

15. Model Summaries for Distress and Posttraumatic Growth

16. Model Summaries for Distress, RCOPE, and Posttraumatic Growth 
17. Path Coefficients Between Distress, RCOPE, and Posttraumatic Growth (Re-Specified Model) (......................................................................... 128

18. Direct and Indirect Effects of the Hypothesized Model................................. 129 


\section{LIST OF ABBREVIATIONS}

$\begin{array}{ll}\text { NRC } & \text { Negative Religious Coping } \\ \text { PRC } & \text { Positive Religious Coping } \\ \text { PTD } & \text { Posttraumatic Depreciation } \\ \text { PTG } & \text { Posttraumatic Growth } \\ \text { PTGI } & \text { Posttraumatic Growth Inventory } \\ \text { PTSD } & \text { Posttraumatic Stress Disorder } \\ \text { RCOPE } & \text { Religious Coping } \\ \text { SARL } & \text { Sexual Abuse by Religious Leaders } \\ \text { THOS } & \text { The Hope of Survivors }\end{array}$




\section{Acknowledgements}

This dissertation is dedicated to my husband, Chris, who has never stopped believing in me or my dreams. Thank you for your steady encouragement, your persistent faith in me, and the incredible sacrifices you have made along the way. It is because of you that I am here. I would also like to thank my children - Ryan, Alicia, and Jasminewho have been my cheerleaders and my inspiration.

I am grateful to all who have encouraged and supported me along this path. I would like to give special thanks to my dissertation committee for their guidance and encouragement through this educational journey. To Dr. Gabriel—-thank you for your dedication, your optimism, and your commitment to my research. I am grateful for your willingness to go above and beyond. To Dr. Kijai — thank you for your guidance through the maze of statistical analyses. Your help has been indispensable, and I am grateful. To Dr. Carbonell — thank you for your keen insight and helpful feedback. Your valuable contribution will not be forgotten.

I would like to thank the participants for the courage to include their voices in this study. Your story matters, and I am thankful that you have chosen to be counted.

Lastly, I would like to thank God, who provided me with the ability, the strength, and the grace to make this dream a reality. 


\section{CHAPTER 1}

\section{Introduction}

Growth through suffering has been an acknowledged theme in the history of many world religions. Indeed, numerous authors, philosophers, speakers, and poets have pondered the notion that unique growth may be experienced in the wake of trial and

adversity (e.g., Angelou, 1969/2015; Frank1, 1959/2006; Nietzsche, 1889/2009). That positive life changes can result from tragic life events has increasingly intrigued researchers in recent years. Many studies have investigated this phenomenon with interesting and varied results. It has been explored among survivors of various tragic/painful events, such as terrorism (Hobfoll et al., 2007); losing a child and other forms of bereavement (Bratkovich, 2010; Taku et al., 2008; Tedeschi \& Calhoun, 2006); traumatic exposure by police officers in the line of duty (Chopko, 2010), intimate partner violence (Bitton, 2014; Cabral, 2010; Cobb et al., 2006), leukemia treatment (Danhauer et al., 2013), childhood sexual abuse (Hartley et al., 2016; Shakespeare-Finch \& de Dassel, 2009), adult sexual abuse/assault (Lev-Wiesel et al., 2005), cardiac surgery (Ai et al., 2013), cancer (Bellizi, 2003; Mosher et al., 2006; Skaczkowski et al., 2013), and war (Powell et al., 2003). The existing literature provides evidence suggesting that, at least for some individuals, certain types of growth are possible in the aftermath of trauma.

The phrase "vulnerable yet stronger" has been used to summarize one aspect of the experience of growth that can occur in the wake of harrowing life events (Calhoun \& 
Tedeschi, 2006, p. 5). In their research, Tedeschi and Calhoun (1996, 2004a, 2004b; Calhoun \& Tedeschi, 1998, 1999, 2004, 2006) found that positive psychological change is possible, following negative life events, despite the adverse psychological consequences that may also ensue after a crisis. Extensive exploration and factor analysis of this paradoxical phenomenon yielded five domain areas in which this growth is likely to occur: (1) personal strength, (2) new life possibilities, (3) relating to others, (4) appreciation of life, and (5) spiritual change (Tedeschi \& Calhoun, 1996).

A review by Zoellner and Maercker (2006) summarized the theoretical models that have had the most significant impact on current conceptualizations of posttraumatic growth (PTG). Outcome models conceptualize PTG as positive psychological change (i.e., outcome) that results from the struggle with highly challenging and traumatic life events (Schaefer \& Moos, 1998; Calhoun \& Tedeschi, 1999). Aldwin et al. (1994) proposed that stressful events may have a transformational function with one of three outcomes: (1) a return to baseline functioning (i.e., homeostatic coping), (2) an increase in functioning to above baseline levels (i.e., growth), or (3) below baseline functioning (i.e., negative coping). O'Leary and Ickovics (1995) offered a comparable model in which the three outcomes are labeled "recovery," "thriving," and "surviving."

Other prominent outcome models have focused on identifying and explaining the mechanisms of PTG. For example, Schaefer and Moos (1998) identified the mediators of growth in the aftermath of crises as the environmental and personal systems that shape a person's response to general life events. These systems include (1) individual resources such as disposition, self-efficacy, health, and drive, and (2) environmental factors such as relationships, support from family/friends, economic circumstances, and social context. 
According to this model, the magnitude, timing, and longevity of the traumatic event affect growth. Schaefer and Moos (1998) assert that "approach coping" is more likely to generate growth than "avoidance coping" in trauma.

PTG, a model of growth-through-suffering conceptualized by Calhoun and Tedeschi $(1998,1999,2004,2006)$, has received a great deal of attention in the literature. In this model, PTG is seen as an outcome variable that results from the severe disturbance of personal worldview and schemas following a devastating event or experience. As deeply held beliefs about the world are disrupted and challenged, the ensuing distress gives rise to persistent rumination as the individual attempts to (1) make sense of the event and (2) reduce distress. Growth is presumed to occur if rumination engages the cognitive processes that drive re-evaluation and meaning making.

In times of high stress, many individuals turn to religious and/or spiritual beliefs as they strive to understand and confront painful life experiences. There is much evidence to suggest that spiritual practices continue to be an essential part of recovery for many individuals who have experienced traumatic events (Kopacz et al., 2016; Levin, 2012; Pargament, 1997; Ronneberg et al., 2016). Access to religious/spiritual resources during stressful events may include the use of religious thoughts, feelings, appraisals, specific spiritual practices such as meditation/prayer, as well as relationships with spiritual individuals, communities, and/or clergy (Pargament, 1997; Pargament et al., 2000). Pargament (1997) has identified this approach to managing stress as religious or spiritual coping. This term encompasses two essential parts: (1) religion/spirituality and (2) coping. 
This study explored PTG in greater detail in a very specific population: female adult survivors of sexual abuse by religious leaders (SARL). It examined the relationships between initial distress, spiritual coping practices, and PTG in this group.

\section{Rationale for the Study}

While the extant literature offers evidence of growth in some cases of trauma, more research regarding the contribution of spiritual coping to PTG will add to this existing body of knowledge. In particular, we know very little about PTG outcomes in female survivors of SARL. The struggle encountered by this very specific population incorporates preexisting spiritual/religious affiliations and assumptions that often add another unique and complex dimension to the experience of abuse. This study contributed uniquely to a deeper understanding of this issue.

\section{Statement of the Problem}

In August 2018, over 300 Catholic priests in Pennsylvania were accused of sexually abusing more than 1,000 children over seven decades (Boorstein \& Gately, 2018). The report brought to the forefront, once again, sexual abuse by trusted leaders in the church community. SARL first received heightened media attention in the latter part of the 20th century as a profusion of high-profile lawsuits were filed against the Catholic Church. Stories published in the Boston Globe in 2002 erupted in a scandal of cleric abuse and a pervasive cover-up, implicating powerful individuals and bringing cleric abuse to the forefront. To many, it appeared to be the birth of a crisis. Yet, despite this increased attention, SARL was not a recent phenomenon.

Before the dissemination of the Globe articles, a significant number of the Catholic clergy had already been implicated in the abuse of boys, women, and girls in 
published accounts both nationally and internationally dating back to 1984 (Marcel, 2013). Between 1950 and 2002, over 10,000 accusations against Catholic clergy came to light (The John Jay College Research Team, 2004 as cited in Folger et al., 2008). A database search found that media accounts in English-language newspapers around the world reported related stories of sex abuse by priests in increasing number from 22 stories in 1985 to 180 stories in 1989, to an average of 777 per year from 1992 to 1998. That average dropped to 500 per year from 1999 to 2001 and then ballooned to 8,905 stories in 2002 when the Boston Globe coverage emerged (Marcel, 2013). In 2002, over 600 stories published in the Boston Globe revealed unprecedented and extensive complicity by prominent clergy in the Catholic Church, uncovering decades of abuse by over 250 priests in the Boston archdiocese alone (2013). As additional cases came to light, the number of clergies and religious leaders accused of sexual misconduct continued to rise in North America and across the globe.

In the 16 years since the articles in the Boston Globe revealed a pattern of sexual abuse by priests, the impact of this abuse on its victims has been the subject of ongoing discourse and study (Benkert \& Doyle, 2009; Doyle, 2009; Farrell, 2009; Pargament et al., 2008; Shea, 2008). While the initial shock of these reports has subsided, and discussion of this topic has become a heartbreaking but familiar theme, survivors continue to live with the psychological fallout of their experiences for many years. The distinctive nature of the abuse suffered by these survivors lies in the unique relationship that existed between the victims and the perpetrator. To a vast majority of survivors, the abuser was a highly respected and revered individual within a trusted religious system, whom many perceived as being very close to God (Farrell, 2009). Frequently adding to 
the perplexity of the trauma is the apparent lack of redress received by the victims who disclosed their abuse (Fater \& Mullaney, 2000; Flynn, 2008; McMackin et al., 2008). The victim experiences the impact of religious trauma on two levels: (1) the psychological/ emotional preparation that increases vulnerability to abuse and (2) a toxic spirituality which determines the shape and effect of the abuse (Doyle, 2006).

Many have referred to the revelations of sexual abuse by clergy as the most devastating crisis facing the Catholic Church in modern times. However, there is much evidence to suggest that the Catholic Church does not confront this issue alone (Bryant, 2010; Goldenberg, 2013). Other denominations with the same problem include the Episcopal church (Richards, 2004), Protestants (Disch \& Avery, 2001), and Jewish faiths (Neustein \& Lesher, 2008).

The present study contended that abuse that occurs at the hands of religious leaders has a unique spiritual quality that contributes to its complexity. However, it is this spiritual dimension or belief system that is often a useful resource to many individuals during major life crises. The experience of abuse may create a chasm between survivors and their assumptive world of spirituality that is not easily reconciled and may cause survivors to distance themselves from spiritual support/resources. This study was interested in exploring whether spiritual coping affected PTG outcome for the survivor, despite the unique qualities and inherent contradictions of their experience.

\section{Purpose of the Study}

The primary purpose of this study was to explore whether spiritual coping mediates the relationship between distress and PTG in female survivors of sexual abuse by clergy. It examined the extent to which spiritual coping affected the outcome of PTG 
in survivors at various levels of distress. This study additionally sought to identify the context of abuse in survivors of SARL and to explore the relationships between distress and PTG and spiritual coping and PTG. The goal was to shed light on these relationships and the mechanisms that facilitate PTG.

\section{Conceptual Framework}

This study will use the model developed by Tedeschi and Calhoun (1996, 2004b) for PTG. In this model, PTG is an outcome variable that results from the severe disturbance of personal worldview and schemas following a devastating event or experience. The model asserts that when deeply held beliefs about the world are disrupted and challenged, the ensuing distress gives rise to persistent event-related rumination as the individual attempts to (1) make sense of the event and (2) reduce distress. Growth is presumed to occur if rumination engages the cognitive processes that drive re-evaluation and meaning making. Challenges to deeply held personal beliefs are a common experience for survivors of SARL.

In this study, abuse by religious authority is the wrongful use of the power acquired through a position of authority in a religious organizational body and a perceived connection with the divine (i.e., God), to exploit, harm, or control a less powerful individual (Bilsky, 2013). Thus, SARL is the misuse of this religious authority to exploit sexually, harm, or control a less powerful person. Folger et al. (2008) developed a unified theoretical model of sexual abuse perpetrated by religious leaders after reviewing current theories of adversity and adaptation. They considered the specific

processes unique to SARL, including (a) improper sexual advances/behavior by religious leaders, (b) the building/nurturing of relationships that facilitate the abuse, (c) the 
spiritual/religious context of the abuse, and (d) the psychological consequences in the aftermath for survivors, and the religious community. The resulting theory incorporated the relational dynamics that exist between the perpetrator, the survivor, and the religious institution/community. These overlapping dynamics are identified as three relational categories: (1) the relationship between the survivor and the abusing leader, (2) the dynamics between the spiritual/religious community and the survivor after the abuse has been revealed, and (3) the dynamics between the perpetrating leader and the community after disclosure.

\section{Theoretical Model of PTG in Survivors of SARL}

Given some current research evidence suggesting that faith-based factors can have a mediating influence on growth following traumatic life events (Ai et al., 2013; Bade, 2000; Gall et al., 2007; Krause et al., 2017; Laufer et al., 2009), the present theoretical model proposed that spiritual coping will mediate distress levels and increase PTG outcomes in survivors of SARL. This study applied the Tedeschi and Calhoun (2006) model of PTG to the experience of female survivors of SARL and hypothesized that survivors who changed life schemas because of the struggle with the harsh realities of trauma and engaged in positive spiritual/religious coping practices would have higher PTG outcomes than survivors who engaged in negative spiritual/religious coping practices. This study further hypothesized that survivors who experienced high levels of distress and engaged in positive spiritual/religious coping would experience higher levels of PTG, as they would be more likely to engage in the cognitive processes that enable change, thus setting the stage for greater growth. 
Throughout life, individuals develop a set of assumptions and beliefs around which they shape their view of the world. For spiritual/religious individuals, these assumptions create paradigms that offer a sense of meaning and provide a lens through which the person's world, environment, and experiences may be understood. They are the backdrop upon which the individual narrative/story is written. The "pre-trauma" functions within this narrative (Calhoun \& Tedeschi, 2006). Sexual abuse in a spiritual/religious context can so convulse the schemas that have provided significance, stability, and meaning to the individual's world that they become severely threatened (Tedeschi \& Calhoun, 2004b). This threat may directly impact the degree of psychological distress experienced by the victim. Tedeschi and Calhoun (2004b) contend that psychological distress occurs to the degree to which the victim challenges these assumptions - particularly expectations about human kindness, predictability, and control. For spiritual individuals, this may include challenges to their relationship with God, their trust in religious institutions and people, their spiritual growth, and their view of the world. The outcome of PTG depends on the critical conflict with the current posttrauma reality.

According to the Calhoun and Tedeschi (2006) model, immediately following a major life crisis, people seek ways to manage the crippling distress that often results from the event(s). Spiritual/religious persons will naturally seek understanding from their pretrauma paradigms. Their attempts to manage the distress will engage automatic cognitive processes that can eventually lead to challenges of their schemas and possible changes that contribute to PTG. In this study, the resulting cognitive engagement in this process will be referred to as "event-related rumination." 
Initially, there are automatic and persistent thoughts, which are intrusive and mostly negative. While victims of SARL may make great efforts to retain their life narratives and systems of belief in place, the more severe the trauma, the greater the distress, and the greater the odds that previous ways of thinking about life will no longer be appropriate given the new information. It cannot be business as usual. Thus begins a prolonged process of disengagement from some of the previous goals and assumptions that shaped their narrative (Tedeschi \& Calhoun, 2004b). Note that it is the distress of SARL survivors that drives this process, as the person seeks to diffuse discomfort.

The journey toward growth includes the search for avenues of relief, through self-disclosure and social support, which provide survivors with pathways for developing alternative narratives that better incorporate the new information (Tedeschi \& Calhoun, 1996, 2004b; Neimeyer et al., 2006). For survivors of SARL, the avenues of relief referenced by the Calhoun and Tedeschi (2006) model, are likely to include religious/spiritual coping activities such as personal prayer and connecting with individuals or groups who can provide spiritual support, encouragement, and/or group prayer. Sharing stories and exchanging thoughts/ideas with other members of their spiritual group offers survivors differing perspectives, opportunities for cognitive restructuring and reattribution, and allows for deeper processing of critical questions and information. The encounters with supportive and like-minded others create a bond with needed allies and force the individual to re-examine and reconstruct stories of meaning and purpose. When this self-disclosure occurs in a spiritual context, survivors may engage in spiritual coping strategies such as: seeking a stronger connection with God, seeking to let go of anger, joining a prayer group, or spiritual journal writing. These 
practices may provide survivors with the supportive space needed to grapple with their experience. For survivors of SARL, this often involves coming face to face with the additional challenge of confronting (1) perpetrators who, in the guise of being a representative of God, used their powers to bring injury to the survivor; (2) a religious organization or system that allowed the abuse to occur; (3) members of the religious body or community of believers who may place blame on the survivor; and (4) God, who may be perceived as not having intervened to protect the survivor from harm.

Self-disclosure and spiritual coping strategies such as those mentioned above lead to more intentional cognitive processing. The role of cognitive engagement through rumination as described in this PTG model may seem to be counter-intuitive, considering the demonstrated link between negative intrusive rumination and depressive symptoms. However, Tedeschi and Calhoun (1996, 2004, 2006) use Martin and Tessler's (1996, as cited in Tedeschi \& Calhoun, 2004b) conceptualization and definition of rumination-the recurrent and persistent thinking that occurs on a conscious level, is related to a particular event or problem, and is readily elicited through an indirect connection with the individual's previous objectives, goals, and experiences. This rumination can include sense making, problem-solving, reminiscence, and anticipation. Tedeschi and Calhoun (2004b) observe that survivors develop a "turning point" narrative of the trauma, into which they separate life before and after the traumatic event. Goals and thinking that were possible before the event no longer seem possible. The process of self-disclosure and support-seeking assist survivors in moving from intrusive rumination to more intentional rumination that involves problem-solving and sense making. This leads to changes/modifications in schemas, life narratives, and spirituality. While successful shifts 
in life schemas lead to PTG, distress may persist and co-exist with growth (Baker et al., 2008).

While a majority of survivors are not thinking about growth in the immediate aftermath of severely distressing events, many value the sense that they have made important gains through their fight for survival. Tedeschi and Calhoun (2004b) found that trauma survivors generally report experiences of growth in the following areas: (a) increased gratitude and a change in priorities, (b) deeper and more meaningful relationships, (c) a sense of greater inner strength, (d) a desire to explore new options for living, and/or (e) a new or greater spiritual development.

To summarize this process using Calhoun and Tedeschi's (2006) PTG model as a guide, the pre-crisis functioning of the women in their study incorporated spiritual/religious beliefs and appraisals in their understanding of the world, others, and themselves. The traumatic experience of SARL left many of these women struggling to manage their emotional distress, challenging their life narratives, and/or questioning previously held beliefs/goals. Rumination following the event(s) is initially negative, intrusive, and persistent as survivors attempt to manage debilitating distress. Selfdisclosure and connection with spiritual others can lead to more intentional rumination or cognitive processing, which may facilitate schema changes, changes in spiritual thinking, and a modified life narrative. Changes that represent better-than-pre-crisis functioning in the domain areas identified by the Posttraumatic Growth Inventory (PTGI; Tedeschi \& Calhoun, 1996) (i.e., increased gratitude and a change in priorities, deeper and more meaningful relationships, a sense of greater inner strength, a desire to explore new options for living, and/or a new or greater spiritual development) suggest PTG. See 
Figure 1 on page 14 for a model of posttraumatic growth adapted from Calhoun \& Tedeschi, 2006.

\section{Research Questions}

The research questions were:

(1) What is the context and nature of the SARL in the sample of female survivors?

(2) What are the effects of spiritual coping on PTG among female survivors of

\section{SARL?}

(3) What are the effects of distress on PTG among female survivors of SARL?

(4) To what extent does spiritual coping mediate the relationship between distress and PTG among female survivors of SARL?

\section{Hypotheses}

This study hypothesized that:

(1) Survivors with positive spiritual coping styles will experience higher levels of PTG than those who use negative spiritual coping styles.

(2) Distress will be positively associated with PTG and survivors with low levels of distress will experience less PTG than those with high levels of distress.

(3) Survivors with high levels of distress and positive spiritual coping styles will experience higher levels of PTG than women with high levels of distress who practice negative spiritual coping. 


\section{Figure 1}

A Model of Posttraumatic Growth Adapted from Calhoun and Tedeschi (2006).

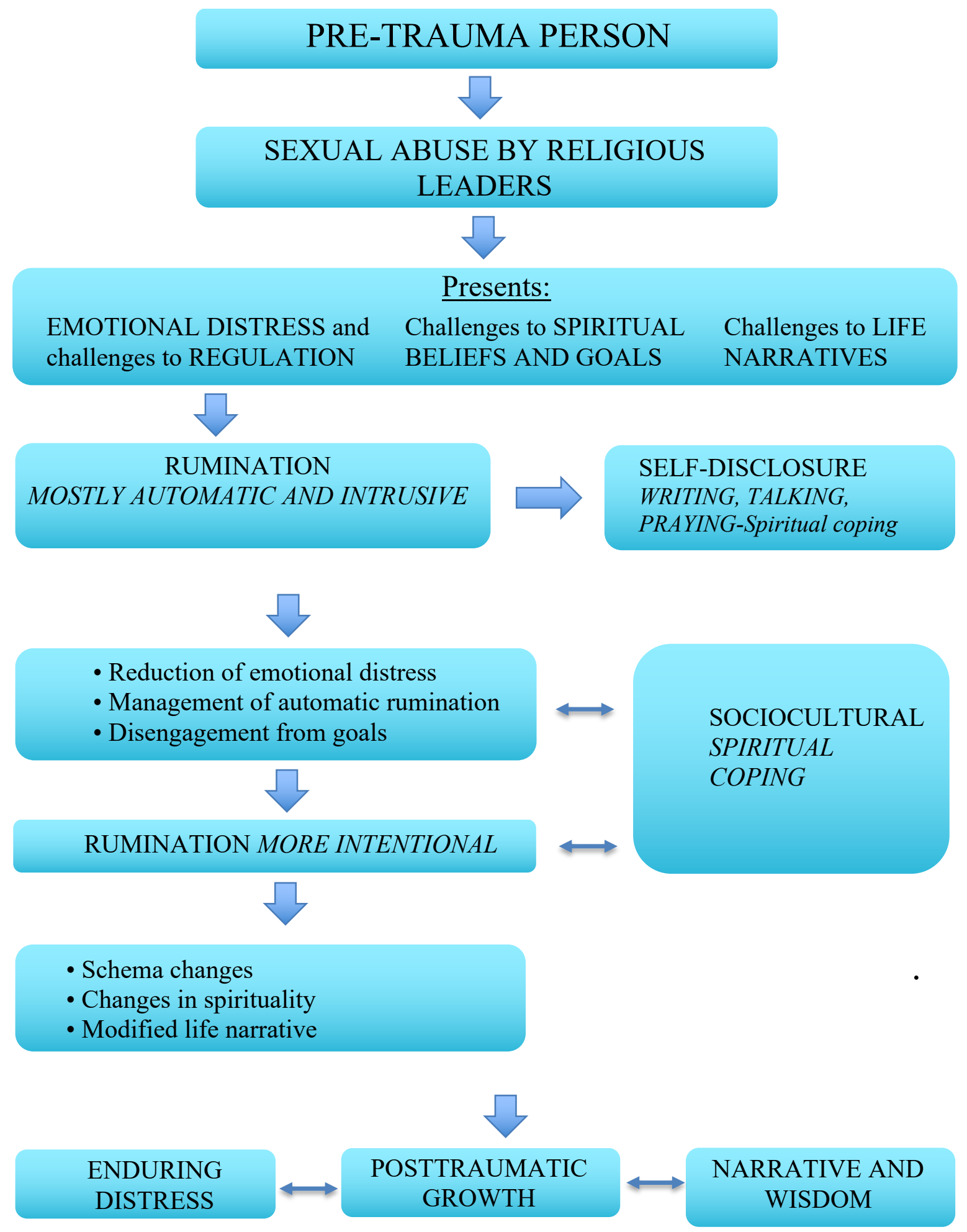




\section{Figure 2}

The Hypothesized Relationship between Distress, Spiritual Coping, and Posttraumatic Growth

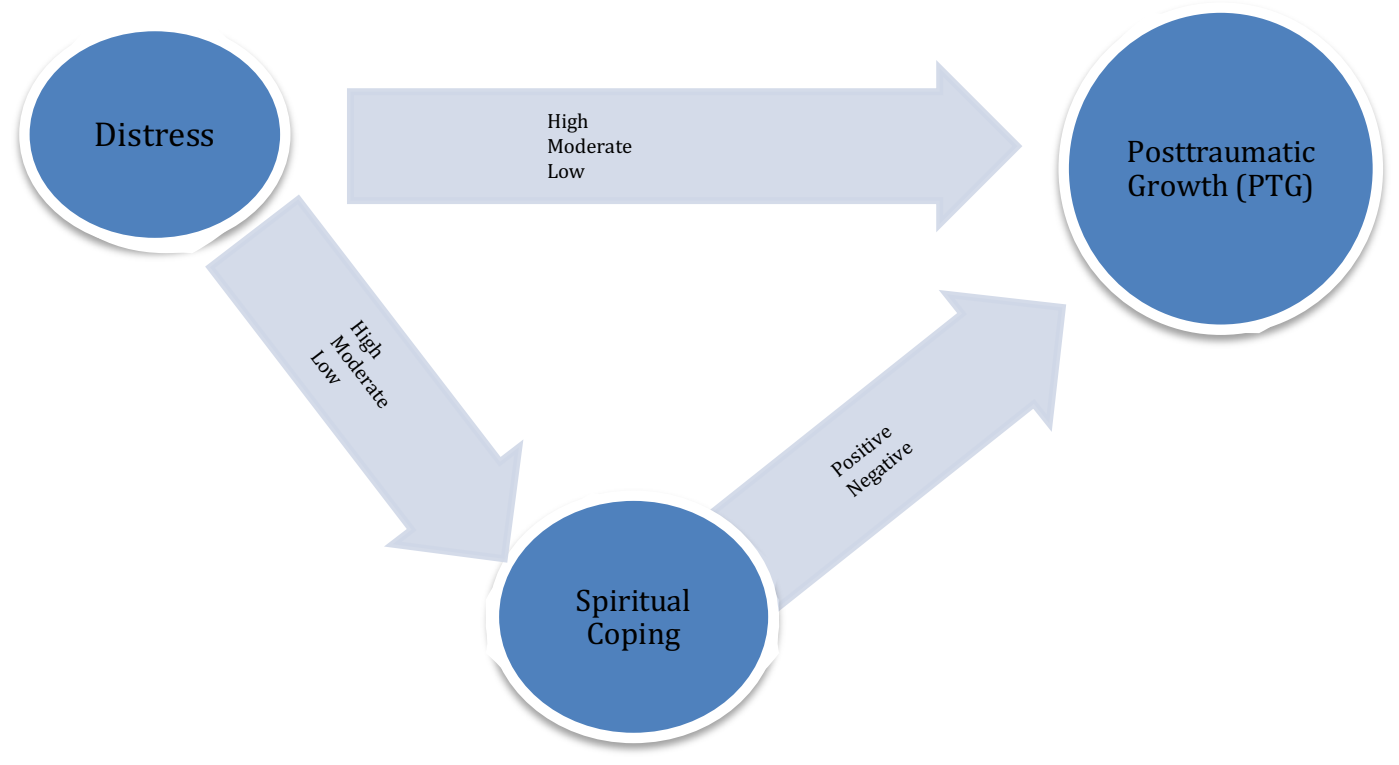

\section{Significance of the Study}

This investigation has potential implications for both empirical and clinical

literature. The impact of sexual abuse and its psychological sequelae is well researched. However, the idea that psychological benefits can occur in the wake of tragic and/or painful events is a burgeoning line of inquiry. Investigating the impact of spiritual coping on PTG among survivors of SARL can contribute to our understanding of the role of these spiritual factors in this population.

In addition, while the construct of PTG has been studied in relationship to bereavement and various other traumatic experiences, including sexual abuse, there is little research revealing the nature of the connection between PTG and sexual abuse perpetrated by religious leaders. And while the limited research on the psychological 
impact of this abuse has found that survivors experience similar psychological sequelae as that of other sexual abuse survivors, the unique spiritual struggles faced by this population have been minimally addressed in the literature. Spiritual struggle is identified as a form of negative spiritual coping (Pargament et al., 2011). The current study helps to shed light on how these unique characteristics of SARL (e.g., survivors' ideas about God, the church, and the sacred) affect PTG outcome.

Another important contribution of this study involves an exploration of the types of spiritual coping that contribute to PTG. Research suggests that positive religious coping is often associated with resilience, deepening of spirituality, and stress-related growth following traumatic experiences (Shaw et al., 2005; Pargament 1997). This study helped to determine whether this holds for survivors of SARL, given the supreme contradictions with which these survivors struggle, and the challenges to their spirituality/religiousness.

This study additionally contributed to the current knowledge-base on the relationship between traumatic distress and PTG outcomes. Presently there are conflicting results in the literature regarding the relationship between these two variables. This study will provide additional evidence regarding the nature of this connection specifically for survivors of SARL.

Previous research has determined that there are specific factors that appear to predict the likelihood of PTG outcomes, such as cognitive engagement and event-related rumination (Calhoun \& Tedeschi, 2006; Calhoun et al., 2000; Cann et al., 2011). This study explored the relationship between distress and PTG, spiritual coping and PTG, and contended that positive spiritual coping styles mediate the relationship between distress 
and PTG. It offers direction for further study into distress and spiritual coping as predictors of PTG in female survivors of SARL, and suggestions for future clinical application.

\section{Definition of Terms}

\section{Distress}

The psychological discomfort or unpleasant emotions that sometimes develop in the aftermath of traumatic events. This definition is based on "The end result of factorse.g., psychogenic pain, internal conflicts, and external stress - that prevent a person from self-actualization and connecting with significant others" (The McGraw-Hill Concise Dictionary of Modern Medicine, 2002). These feelings impact/interfere with daily functioning and can cause negative views of self, others, and the environment. This study will use the terms "psychological distress" and "distress" interchangeably.

\section{Event-Related Rumination}

The "cognitive processing" involved in the recurrent/persistent focus of attention and thoughts toward a specific life event. Research suggests that significant cognitive engagement around a traumatic event is necessary for the process of PTG to occur (Tedeschi \& Calhoun, 2004b). It may involve remembering, anticipating, problemsolving, resolving, or attempting to make sense of what has occurred (Martin \& Tesser, 1996 as cited in Calhoun et al., 2000).

\section{Meaning Making}

"A cognitive and affective change in the way an individual perceives a painful experience" (Marrotta-Walters, 2015, p. 64). Meaning making may be present at any 
point in the healing process and is perceived to be an integral part of spiritual growth (Gall et al., 2005).

\section{Posttraumatic Growth}

"The positive psychological change experienced as a result of the struggle with highly challenging life circumstances" (Tedeschi \& Calhoun, 2004b, p. 1) This growth may be evidenced in various ways, including increased gratitude and appreciation for life, deeper interpersonal relationships, a sense of greater inner strength, a re-adjustment of priorities, and/or a fuller spiritual/existential life. PTG is identified as growth that surpasses the previous functioning in one or more of the mentioned areas.

\section{Sexual Abuse by Religious Leaders}

The wrongful use of the power acquired through a position of authority in a religious organizational body and a perceived connection with the divine (i.e., God), to exploit, harm, control or shame a less powerful individual (Bilsky, 2013). By extension, a definition of SARL must include excessive authority, the misuse of power associated with that authority, and the inappropriate use of the divine/God (Bilsky, 2013).

\section{Spiritual Coping}

The use of religious and/or spiritual activities to confront, grapple with, and address highly stressful life occurrences (Pargament et al., 2005). This includes a variety of activities that differ according to faith traditions and cultures, such as prayer (individual, intercessory, or group prayers), meditation, practicing forgiveness, singing songs of praise/worship, spiritual re-appraisals, connecting with a community of believers (2005). Positive spiritual coping practices reflect a secure relationship with God, a sense of connectedness with others, and meaningful attributions to life. Negative spiritual 
coping practices reflect punishing God reappraisals, demonic reappraisals, spiritual discontent, interpersonal religious discontent, and pleading for direct intercession (Pargament et al., 1998).

\section{Spirituality}

"A search for the sacred" (Pargament, 1999, p. 12) in the path toward significance and meaning. "Sacred" refers to a supreme being/higher power, God, and/or transcendence, as well as the objects associated with this conception $(1999$, p.12).

\section{Delimitations}

The present study sheds light on the types of spiritual coping that promote PTG in survivors of SARL and is limited in its application to this specific population. The author only examined responses from the perspective of female survivors. Hence, our ability to apply the results of the study to male survivors of SARL is limited.

\section{Limitations}

The population sample for this study was obtained from a single organization that deals most prominently with women of Christian faith traditions and may inadvertently exclude individuals who identify with non-Christian faiths. Thus, the results are limited in their generalizability to survivors of SARL who identify with non-Christian religions and/or spiritual practices.

Additionally, due to the nature of the organization from which the sample was taken, individuals who did not subscribe to any faith or spiritual tradition are not represented in the sample. While this limits our ability to generalize the results of this study to non-religious/spiritual persons, spiritual coping practices are not likely to be an option of choice for those individuals. 
The outcome of this study has similar limitations to studies utilizing self-report measures. Also, the data were obtained from surveys voluntarily returned by participants and may thus reflect a self-selection bias.

\section{Organization of the Study}

This research contains five chapters. The first chapter introduced the topic under study, providing a background of the problem and a conceptual framework. Chapter 2 reviews the current literature on PTG, spiritual coping, and distress among female survivors of SARL. Chapter 3 described the methodology used for this study and covers research questions and design, instrumentation and data collection, and data analysis.

Chapter 4 reported the results of the statistical analyses of the data with associated figures and tables. Chapter 5 summarized the findings of the study, discussed the results in terms of previous research, and drew conclusions. 


\section{CHAPTER 2}

\section{LITERATURE REVIEW}

\section{Introduction}

In recent years, an emphasis on positive psychology and strengths-based personal resources has mobilized research examining the phenomenon of growth in the aftermath of adversity. That growth can result from crises and debilitating life experiences is not new. Human suffering has long been viewed as a catalyst for growth. This timeworn notion is present in art, philosophy, literature, and folklore. Yalom and Lieberman (1991) and Frankl (1956/2006) were among the early researchers who explored the concept of growth through adversity, offering a scientific foundation in modern times.

The change/growth processes observed in survivors of trauma have been conceptualized in a variety of ways (Park et al., 1996; Tedeschi \& Calhoun, 1996), resulting in subtle differences and diverse nomenclature (e.g., PTG, stress-related growth, thriving, flourishing). Of these conceptualizations, PTG and its associated theory as proposed by Calhoun and Tedeschi $(1996,2006)$, has been the most prominent. This review explored the current research on PTG and examined the evidence for the relationships that exist between psychological distress, spiritual coping, and PTG. This research has proposed that spiritual coping mediates the relationship between 
psychological distress and PTG such that positive spiritual coping will increase the PTG outcomes in individuals with high levels of distress due to trauma resulting from SARL.

The literature review falls into two segments. The first discusses PTG and proceeds as follows: (1) a scientific and theoretical overview of PTG and then discusses predictors and facilitators of PTG in the literature and religious/spiritual coping as a correlate of PTG; (2) an examination of the existence of PTG among female survivors of sexual abuse; (3) psychological distress as correlates; (4) a scientific overview of psychological distress; (5) psychological distress in relationship to spirituality and spiritual coping; (6) psychological distress among female survivors of sexual abuse and female survivors of SARL; (7) coping mechanisms utilized by survivors of SARL.

The second segment explores coping among survivors of sexual abuse and female survivors of SARL and proceeds as follows: (1) the concept of spiritual coping and the relationship of spiritual coping to PTG, (2) the significance of spirituality among survivors of SARL; and (3) the relationship between spiritual coping, psychological distress, and PTG among female survivors of sexual abuse and SARL. This chapter concludes with a summary and analysis of the literature review.

\section{Posttraumatic Growth: An Overview}

In 2004, Tedeschi and Calhoun defined PTG as "the positive psychological change experienced because of the struggle with highly challenging life circumstances," (Tedeschi \& Calhoun, 2004b, p.1) Research suggests that PTG results from the challenges to assumptive worldviews that often occur when individuals are confronted with the bewildering and distressing realities of a traumatic experience (Tedeschi \& Calhoun, 2004b; Calhoun \& Tedeschi, 2006). Deeply disturbing events produce negative 
emotions that activate the cognitive and psychological processes involved in managing the associated distress. It is through these very difficult processes that researchers believe positive transformation can occur.

While traumatic life events commonly generate troublesome psychological responses, a concurrent process, stimulated by the high levels of distress and the individual's attempt to adapt, can stimulate a unique growth. There is evidence to suggest that while a small percentage of persons experiencing traumatic life events develop severe psychological disorders, a far greater number of these individuals reported experiences of positive growth in the aftermath of trauma (Tedeschi \& Calhoun, 2004b). Nevertheless, researchers caution that growth is not the inevitable result of trauma, nor does it eliminate trauma-related distress. Instead, growth seems to occur alongside ongoing distress.

Tedeschi and Calhoun $(1996,2004 b)$ used the term "posttraumatic growth" to depict the specific qualities of a growth experience distinct from other related terms in use in current literature. For example, they argue that the term stress-related growth (Park et al., 1996) does not fully capture the intensity of the PTG process, as it may include lower level stressors. The potential for growth, they contend, is directly related to the power of the stressor to challenge existing life schemas. These researchers additionally argue that many of the changes resulting from PTG were not merely illusory, as suggested by Hobfoll et al. (2007). Research has found that there are measurable and life-transforming changes that have occurred in the lives of individuals who experienced PTG (Tedeschi \& Calhoun, 2004b). Thus, terms that emphasize the "illusion" of change were also unsuitable. Further, PTG is conceptualized as a process involving continuity 
and progression, rather than being identified as one of several means of coping (Calhoun $\&$ Tedeschi, 2006). Researchers also suggest that PTG occurs in severe trauma in which individuals have faced significant threats or life-shattering events and are moved to reexamine fundamental life schemas in the midst of psychological distress. That distress is essential to PTG is at odds with the concepts of thriving or flourishing. Growth that occurs during this distress will exceed the level of previous functioning and, as such, is not simply a return to baseline.

Tedeschi and Calhoun (2004a) also examined the distinctions in the literature between PTG and such concepts as resilience, optimism, hardiness, and sense of coherence. Resilience has been identified as the ability of individuals to adapt, integrate healthy functioning over time, and move forward with purpose in spite of harrowing life events (Southwick et al., 2014), while hardiness has been represented as the tendency to meet difficult circumstances and make changes beneficial to personal growth (Kobasa, 1979; Kobasa et al., 1985). Optimism refers to an attitude of positive expectation (Scheier \& Carver, 1985), and a sense of coherence was identified as the ability to comprehend, manage, and find meaning in traumatic events (Antonovsky, 1993), which may provide benefits to the survivor. By comparison, PTG is intended to capture specific changes that go beyond pre-crisis functioning. Thus, resistance to psychological damage alone does not necessarily indicate the existence of PTG. There is a qualitative transformation in functioning that is missing in the concepts of resilience, hardiness, optimism, and a sense of coherence that distinguishes PTG. Authors Tedeschi and Calhoun (2004b) contended that severe mental conflict following a traumatic event is a necessary component of the PTG process. 
Tedeschi and Calhoun (1996) and Woodward and Joseph (2003) identified PTG as a multidimensional construct comprising several areas of potential growth that develop differently in each survivor. In a qualitative study, Woodward and Joseph (2003) obtained a sample of 29 individuals through advertisements in the newsletter of the National Organization for People Abused in Childhood. Of this sample, 24 participants were female and five were male, ranging in age from 22 to 72 years. Using thematic analysis of participant narratives, Woodward and Joseph identified 10 areas in which posttraumatic positive change occurred: (1) will to live, (2) awakening of responsibility, (3) validation and acceptance, (4) Love and nurturing, (5) liberation and freedom, (6) mastery and control, (7) belonging and connection, (8) changes in self-perception, (9) gaining new perspectives on life, and (10) changes in relationships. These they divided into three domains: Inner Drive Toward Growth, Vehicles of Change, and Psychological Changes. While their sample was small, the qualitative design of this study allowed for a deeper exploration of the dimensions for potential change.

Tedeschi and Calhoun (1996) developed their model by reviewing the body of literature where positive outcomes of adversity had been studied. A list of 34 items describing the possible growth areas, as identified by individuals with experiences in trauma, was created and worded in a positive direction. Using a 6-point Likert scale, the items were administered to 604 undergraduate students (199 males and 405 females) from a major southeastern university who had reported a significant negative life event in the past five years. Some distressing events reported by students were bereavement (36\%), injury due to an accident (16\%), separation/ divorce (8\%), relationship break-up (7\%), victim of a crime (5\%), academic problems (4\%), unwanted pregnancy (2\%). A 
principal components analysis was conducted on the data and 21 items loading on five factors were retained in the final measure. These factors were labeled (1) Relating to Others, (2) New Possibilities, (3) Personal Strength, (4) Spiritual Change, and (5) Appreciation of Life. These became the five subscales of the PTGI. Internal consistency of the resulting measure was $\alpha=.90$. Test retest reliability over two months was an acceptable $r=.71$. In their sample, women $(M=75.18, S D=21.24)$ reported significantly more benefits than men $(M=67.77, S D=22.07)$ on every factor with the exception of New Possibilities.

The PTG model by Calhoun and Tedeschi (2006) suggests that when survivors move from intrusive to intentional rumination, the cognitive processing of events leads to changes in spirituality and life narratives (Taku et al., 2009). It is at this point that growth and positive changes may be observed. However, survivors may continue to experience concurrent distress. The PTGI (Tedeschi \& Calhoun, 1996) identifies these dimensions as: Relating to Others (i.e., enhanced relationships and interpersonal behavior), New Possibilities (i.e., a perception of new life possibilities), Personal Strength (i.e., a new awareness of competence/capability), and Spiritual Change (i.e., an awakened spirituality or change in belief system), and Appreciation of Life (i.e., increased gratitude and valuing of life).

Nevertheless, interesting contradictions seem to exist concurrently in the survivor's experience, such as individuals who experience greater vulnerability while reporting a stronger sense of confidence in their ability to overcome trials. Some individuals may seek to fulfill their need to talk about the event(s) by building connections and nurturing interpersonal relationships. Enhanced relationships can lead to 
an increased level of compassion for the experiences of others. Questions about life, death, and meaning may become more central as individuals struggle to comprehend and synthesize their experiences into their own personal narratives. Often people will place greater value on the "little things" of life, while re-evaluating their own spiritual and existential assumptions about the world. It is in wrestling with these questions, during painful sequelae, that a reorganization of the life schema can occur.

A phenomenological study by Walker-Williams et al. (2013) examined the relationship between coping behaviors and PTG in 10 South African female survivors of childhood sexual abuse, ranging in age from 23 to 48 years. The participants were $70 \%$ white. The researchers also examined the long-term effects of coping behaviors on their psychological well-being. The participants in this study had taken part in previous research conducted by Walker-Williams et al. (2013) and had received therapy. Inclusion criteria were positive coping behavior, PTG, features of psychological well-being, childhood sexual abuse, disclosure of abuse, and past therapeutic care. Data was gathered using semi-structured interviews and themes were identified via interpretive phenomenological analysis. They found that survivors of childhood sexual abuse who experienced PTG used positive coping behavior and psycho-socio spiritual resources to cope with psychological distress in the aftermath of abuse. For many individuals, this included a renewed personal connection with God, spiritual/religious coping, learning how to regulate their emotions, developing a more optimistic explanatory style, cognitive restructuring, and social support. The rebuilding aspect of PTG involved a daily conscious choice by the survivor to engage in the recovery process. In addition, WalkerWilliams et al. found that the painful and gradual restructuring occurred through the 
eliminating, drafting, and adding of new dimensions to the personal narrative of existing life schemas, such as a realization of power and efficacy, and themes of forgiveness and gratitude. While this study offered insight into the growth possibilities for female survivors of childhood sexual abuse from an educated urban sample who had received therapy, a less educated rural sample might differ in outcome.

\section{Predictors of Posttraumatic Growth}

Fetty (2016) utilized an exploratory method to sketch a theoretical model for

PTG. The researcher used a structural equation model to examine the predictive ability of belief in ultimate justice, optimism, and supportive relationships on PTG and distress outcomes, as well as supportive spirituality, meaning making, and problem solving as mediator variables in female survivors of sexual assault. The goal of this study was (1) to test the theoretical framework of Schaefer and Moos (1998) and (2) to test the factor structure and internal consistency of the English version of the Belief in Immanent Ultimate Justice Scale (Maes, 1998a, 1998b as cited in Fetty, 2016). The sample of 217 had a mean age of 27.5 years and was $73.3 \%$ White American, $16.1 \%$ African American, 6.5\% Latino, $1.8 \% \mathrm{bi}$-racial/multiracial, $1.4 \%$ Native American, and $1 \%$ unspecified. Supportive relationships emerged as a strategy of appraisal and coping, as did problem solving, optimism, and meaning making. Fetty also found that the variables of appraisal and coping (i.e., meaning making, optimism, supportive relationships, and problem solving) mediated the relationship between survivors' worldviews (i.e., supportive spirituality, belief in ultimate justice, and belief in an immanent just world) and PTG/distress outcomes. Her findings bolster previous research that suggests that the existence of supportive relationships is linked to (a) the variables of optimism, problem 
solving, and meaning making (Borja et al., 2006; Orchowski et al., 2013; Schnell \& Becker, 2006; Ullman, 1999) and (b) PTG outcome (Carver et al., 2010; Prati \& Pietrantoni, 2009). While the measures used in this study were found to have good validity and reliability, as a cross-sectional, non-experimental design, causality cannot be assumed nor can all underlying variables be accounted for.

Grad and Zeligman (2017) examined the role of "social interest" and "meaning in life" as contributors to PTG outcome using the following measures: Early Trauma Inventory Self-Report-Short Form, BASIS-A Inventory to measure social interest, PTGI, and the Meaning in Life Questionnaire. A sample of 531 undergraduate students attending an urban university participated in this study. Participants ranged in age from 18 to 56 years $(\mathrm{M}=28.3)$, were mostly female $(61.9 \%)$, and had the following racial/ethnic breakdown: 45\% African American, 20.7\% Asian, 19.1\% White, 8.1\% Hispanic, 2.8\% Middle Eastern, 2.4\% Multiracial, 1\% American Indian, and 1.7\% other racial identity. Many were first generation college students (43.5\%). Grad and Zeligman (2017) identified social interest as a sense of belonging and connectedness to others. Meaning in life was defined as having a sense of connection and purpose in living (Grad \& Zeligman, 2017; Frankl, 1959/2006). They found that meaning in life and social interest were positively related. Meaning in life was also found to be a robust predictor, while social interest was a significant predictor of PTG. Their findings were consistent with previous literature suggesting that social interest and meaning in life are positively associated with PTG (Linley \& Joseph, 2004; Steger et al., 2015). The diversity and size of the sample in this study $(\mathrm{n}=531)$ and the acceptable validity and reliability of the 
instruments used, contribute to its strengths. However, the cross-sectional design limits the ability to infer causality.

For example, in a hierarchical regression analysis, Leeman et al. (2015) found that coping resource effectiveness, social interest, and being cautious significantly predicted PTG outcome in a sample of 143 adults between 18 and 65 years of age who had experienced at least one lifetime traumatic event. The sample in this study comprised undergraduate students attending two urban universities in the southeastern United States and was predominantly female (84\%). Racial/ethnic breakdown was as follows: $33.6 \%$ Caucasian, 34.3\% African American, 11.9\%Asian/Pacific Islander, 7.7\% Black/NonAfrican, 4.9\% Mixed Race, 3.5\% Hispanic, 1.4\% Latino, 0.7\% East Indian, 0.7\% Middle Eastern, and $1.4 \%$ other ethnicity. Of the participants in this study, $65.7 \%$ had no diagnosis, $13.3 \%$ were diagnosed with depression, $8.4 \%$ with anxiety, 5.6\% with ADHD, $2.8 \%$ with bipolar disorder, $1.4 \%$ with PTSD (posttraumatic stress syndrome), and 2.8\% with another diagnosis. The outcome of this study was strengthened by the ethnic diversity of the sample, the strong validity and reliability of the measures employed, and the representation of various mental health concerns within the sample. However, the sample was primarily female and further exploration with male subjects may be needed.

In a smaller study, Dursun et al. (2016) examined meaning in life, vitality, and perceived social support in PTG outcomes among 57 survivors of the 2013 Colorado floods. Individuals were invited to participate in an online survey via social network websites and public outreach recovery teams for flood survivors in Colorado. Of the 121 individuals who accessed the survey website, 57 (25 males and 32 females) completed the scales and this number made up the sample. The characteristics of the respondents 
were: mean age of $39.63(\mathrm{SD}=5.67) ; 83.9 \%$ White, $50 \%$ married, $26.8 \%$ single or never married; $48.2 \%$ college-educated, $16.1 \%$ some college education, and $19.6 \%$ had a master's degree. Respondents identified as $66.7 \%$ non-religious and 33.3\% Christian. Religious affiliation in this sample deviates from the national norm in the U.S. but is consistent with the areas affected by the floods. The search for meaning and perceived social support were robust predictors of PTG in this group (2016). While the sample in this study was small, acceptably valid measures and procedures were used, contributing to the validity of the study. However, the sample was more highly educated and less religious than the general population in the U.S., limiting generalizability.

\section{Posttraumatic Growth, Cognitive Processing, and Event-Related Rumination}

The process of PTG necessitates a re-examination and reorganization of core beliefs as a precursor. The severity of the experience creates challenges to the views about the world. Thus, to reconcile the experience with their assumptions about the world, the individual goes through a process of cognitive engagement, also referred to as rumination and/or cognitive processing (Tedeschi et al., 2007).

Tragedy and bereavement create an environment where survivors often struggle with the disruption of held worldviews (Webster \& Denng, 2015). As survivors attempt to rebuild their lives, the cognitive processing that occurs in the wake of tragedy becomes essential to growth (Greenberg, 1995; Helgeson et al., 2006). Models of cognitive processing in the aftermath of trauma suggest that higher levels of engaged rumination create fertile ground for growth and has been found to enhance the experience of PTG (Calhoun \& Tedeschi, 1998; Helgeson et al., 2006). 
In a cross-sectional, correlational study, Calhoun, Cann, Tedeschi, and McMillan (2000) sought to explore how religious orientation and rumination were related to PTG and the degree to which religious involvement affected PTG outcomes. The following two domains of cognitive processing were examined in relationship to PTG: (1) eventrelated rumination and (2) quest oriented religious beliefs. The sample for this study consisted of 54 young adults ( 35 females, 19 males); mean age of 22.5 years; mostly single (85\%); $80 \%$ Caucasian, $13 \%$ African American, and 7\% Asian or Native American. The participants reported various traumatic experiences: being the victim of a serious crime $(N=4)$; sudden death of a loved one due to an accident, homicide, or suicide $(N=10)$; motor vehicle accident resulting in serious injury $(N=12)$; injury or major property loss due to disaster $(N=2)$; home evacuation because of serious hazard $(N=1)$; and a miscellaneous category (e.g., combat, earthquake, gang-related violence, etc.) $(N=18)$. A standard multiple regression analysis found that higher levels of rumination immediately following the traumatic event enhanced PTG. However, eventrelated rumination that consisted largely of intrusive negative thoughts and that persisted for prolonged seasons were predictive of higher levels of distress and lower levels of growth. One aspect of quest oriented religious beliefs was found to impact growth: openness to religious change. The Quest Scale "was designed to measure ... the degree to which an individual's religions involves [a] ... responsive dialogue with existential questions" (Batson et al., 1993 as cited in Calhoun et al., 2000, p. 523). Included are items such as, "As I grow and change, I expect my religion to grow and change" and "Questions are far more central to my religious experience than answers." While the 
instruments used in this study were adequately valid and reliable, the size of the sample was small, limiting statistical power.

To better understand the transformation process that occurs in PTG (i.e., the positive changes), Cann et al. (2010) determined that it would be valuable to solicit information from study participants regarding both the negative and the positive changes they experienced in the wake of trauma. Cann et al. identified positive changes as PTG, while labeling negative changes as "posttraumatic depreciation" (PTD). Each of the 118 college students in their sample had experienced a traumatic or highly stressful event within the previous three years, such as the loss of a loved one, severe personal injury/illness, being physically assaulted or robbed, and/or military deployment. PTG and PTD were found to be completely unrelated. However, rumination was related to both PTG and PTD, while recent deliberate rumination and recent intrusive rumination were negatively related. They found that deliberate rumination was predictive of PTG, whereas intrusive rumination was predictive of PTD. Thus, the researchers suggested that there was a qualitative difference between deliberate and intrusive rumination, leading to different outcomes (2010). The quality of this study is enhanced by the valid and reliable instruments used to measure the variables as well as the adequate size of the sample. However, as a correlational study, direction of effects cannot be positively determined. In another study supporting the distinctive features of deliberate and intrusive rumination, García et al. (2017) explored the relationship between four types of rumination (i.e., brooding, reflection, intrusive, and deliberate) and stressful life events using confirmatory factor analysis. Of the 750 adult participants 504 (54\% male) who had experienced a highly stressful event in the last four years were randomly selected through 
the electoral roll in Concepcion, Chile. The other 246 participants were recruited from a list of individuals who had recently suffered a workplace injury requiring medical attention and had more than 10 days of sick leave. Respondents ranged in age from 19 to 83 years with a mean age of $38.66(\mathrm{SD}=13.56)$. On average, the time that had transpired since the stressful event was $12.54(\mathrm{SD}=11.99)$ months. Participants were contacted via telephone or through home visits. The study found that deliberate rumination was the most successful predictor of PTG six months after the event in this sample. However, intrusive rumination was a significant predictor of posttraumatic stress symptomatology. The large, randomly selected, sample in this study provides for greater statistical power. Additionally, the satisfactory validity and reliability of the instruments used to measure the variables lends to soundness of the results. However, the study did not control for the time elapsed since the distressing event occurred or for PTSD.

\section{Posttraumatic Growth, Narratives, and Meaning Making}

For PTG to occur, several additional elements must also be in place (Calhoun \& Tedeschi, 2006). First, a severely devastating event must produce a strong emotional response that will cause the fracturing of the assumptive world and will include both emotional and intellectual grappling with new data about the world. Additional factors that appear to influence PTG include the aversive power of post-trauma cognitions (Calhoun \& Tedeschi, 2006), the power of distress to initiate cognitive engagement (Tedeschi \& Calhoun, 2004), personality factors (Tedeschi \& Calhoun, 1996), sociocultural elements or constraints (Calhoun \&Tedeschi, 2006; Pals \& McAdams, 2004; Park \& Lechner, 2006), disclosure and the life narrative (Neimeyer, 2004; Neimeyer et al., 2006). 
The PTG process occurs when trauma is powerful enough to cause persons to challenge and even change previously held, meaning-making assumptions about the world, others, and themselves (Tedeschi \& Calhoun, 2004). These assumptions about the world are identified as narratives, and provide a framework from which goals, meaning, and identity are derived. Pals and McAdams (2004) contend that life narratives are a main contributor to identity and are the framework for connecting all the pieces of the personal puzzle. Thus, they reason that a challenge to the personal narrative is a challenge to the core identity. Life narratives, they argue, are a key to PTG. The ability of individuals to (1) recognize, acknowledge, and explore the personal impact of traumatic events and (2) form a narrative that incorporates a cogent and positive outcome, has emerged as a reliable predictor of PTG.

In a case study, Vilencia et al. (2013) found that meaning making was an essential component of readjustment and growth after trauma. While this study examined the experience of only two women, the qualitative design provided deeper insight into their experience of growth. Vilencia et al. (2013) documented the experience of two adult female survivors of childhood sexual abuse, in an effort to explore the role of meaning making on the path to PTG. The women were recruited from a larger study in which the authors were exploring the journey toward healing in this population. Vilencia et al. (2013) discovered that an essential part of the growth process occurred when the women began to understand the beliefs they had about themselves, others, and the world around them. Turning points for healing began when the women felt compelled to confront the reality of their experience, after having spent many years depleting their personal and emotional resources attempting to cope through avoidance and dissociation. Previous 
research shows that avoidant coping is a strong predictor of depression and poor adjustment (Bal et al., 2003; Brand \& Alexander, 2003; Futa et al., 2003; Wright et al., 2007). The women in the study could experience growth by first gaining an understanding of how their beliefs were connected to their trauma (Vilencia et al. (2013). This process involved deliberate, active rumination as the women examined the impact of the sexual assault and attempted to understand their existing assumptive world. For the women in this study, the agonizing confrontation involved coming face to face with an understanding of their inner worlds and the harmful self-concepts that aided in maintaining their self-destructive feelings and behaviors. Deliberate and critical examination of their core beliefs, with the guidance of a counselor, assisted in the deconstructing and reconstructing the meanings behind their stories, leading to a renewed self-perception. One of the two participants described how this process unfolded for her: It was really about healing and coming up with different ways of being in the world, and different ways of being. It was like "Oh God, this terrible thing has happened to me," it was just a gradual unpicking of all parts of my life that had been built up around having been raped and looking at them one by one and seeing if I wanted to keep them. I got to value the survival skills I put in place but also say goodbye to them as being unnecessary in a really slow way. And it was hard. I remember sessions where most of the session was just working through the anger I felt over everything and everyone. (p. 47)

Vilencia et al. (2013) concluded that the inner world transformations experienced by the women in their case study occurred through a process that included becoming 
aware of their core beliefs, understanding the formation of these beliefs, why they were once protective, and why they are no longer necessary. This cognitive engagement is referred to as deliberate rumination and is distinct from the automatic intrusive rumination that is frequently associated with increased depression and anxiety. Thus, rumination can be deliberate or intrusive (Taku et al., 2008; Taku et al., 2009; Tedeschi \& Calhoun, 2006).

In a mixed methods study, Wright et al. (2007) explored the coping practices, benefits attributions, and meaning-making attempts in 60 adult female survivors of childhood sexual abuse. Participants for this study were recruited via multimedia publicity (e.g., flyers in pediatrician's offices, grocery stores, laundromats, libraries, bookstores, etc.) requesting voluntary, anonymous participation of adult mothers who had experienced childhood sexual abuse. Seventy-nine women requested the mail-in questionnaire, and 60 were returned. The sample was predominantly Caucasian $(91.7 \%)$ with a mean age of 38.83 years $(\mathrm{SD}=8.6)$. The education level ranged from partial high school to doctoral degree, with an average education attainment of partial college. These mothers had an average of 2.1 children and most were married $(76.7 \%)$ or in a domestic partnership (6\%). This study revealed the severe struggle survivors faced when attempting to reconstruct shattered personal schemas in the aftermath of trauma. They found that (1) avoidant coping strategies accounted for $54 \%$ of the variance in depressive symptoms/poor trauma resolution (2) perceived benefits were also frequently accompanied by negative changes, and (3) while many (29\%) were unable to derive meaning from the experience, those who did so (87\%) experienced less isolation. The relatively small sample size of this study decreased its statistical power and should be 
used in connection with larger, more diverse studies in order to further support its findings.

\section{Posttraumatic Growth and Religious/Spiritual Coping}

Spiritual coping has been defined as the use of religious and/or spiritual activities as a means of confronting, grappling with, and addressing highly distressing life events (Pargament et al., 2005). In a study intended to validate a measure of religious coping methods Pargament et al. (2000) found that religious and/or spiritual coping practices yield both effective and ineffective results. The researchers used two samples and confirmatory factor analysis to identify factor structures. One sample consisted of 540 college students who were $93 \%$ white, $69 \%$ female, and averaged 19 years of age. The second sample was made up of 551 elderly hospital patients who were $62 \%$ white, $52 \%$ male, and averaged 68.4 years of age. Regression analyses found distinct variance between types of religious coping methods. Pargament et al. identified positive religious coping practices as those that reflect a secure relationship with God, a sense of connectedness with others, and meaningful attributions to life. Negative religious coping practices were identified as those with punishing God reappraisals, demonic reappraisals, spiritual discontent, interpersonal religious discontent, and pleading for direct intercession. In the sample of college students, practices identified as negative religious coping were associated with decreased physical and mental health. In both samples, positive religious coping was connected to stress-related growth and increased/stronger spirituality. The size of the samples and the procedures used in these studies contribute to their validity. However, the lack of diversity in the college sample would suggest that further studies may be needed for generalizability to diverse populations. 
Numerous studies provide support for the mediatory role of positive religious coping on the path to PTG, following distressing life events. One example of this was a study by Ghafouri et al. (2016) in which the relationship between marital adjustment and PTG was mediated by positive religious coping in couples struggling with infertility. A significant positive relationship between positive religious coping and PTG was also confirmed. In this study, a convenience sample of 250 individuals at an infertility clinic in Tehran, Iran, completed measures for marital adjustment, PTG, and religious coping strategies. The sample included 70 men (28\%) and 176 women (70\%), with and average age of 35 for men and 30 for women. The data was analyzed for correlations using structural equation modeling. The measures used in this study were appropriately valid and reliable, adding to the integrity of the outcome. However, directional affects cannot be conclusively determined due to the correlational nature of the study. In the context of natural disasters, posttraumatic growth appeared to be enhanced by the use of positive spiritual coping (Wlodarczyk et al., 2016). For caregivers of childhood cancer survivors, positive spiritual coping and optimism predicted the ability to find positive outcomes in the face of adversity (Gardner et al., 2017). Adaptive spiritual coping, as compared to spiritual struggles and non-spiritual coping, also seemed to be a distinctive resource in dealing with the psychological adjustment to divorce and was associated with greater PTG in a sample of adults (Krumrei et al., 2009).

In the context of sexual abuse, Fallot and Heckman (2005) examined the extent to which a group of female survivors of childhood abuse and childhood sexual violence use positive and negative religious coping. In this study, two racially diverse samples consisting of a total of 666 women with co-occurring mental health and substance use 
disorders were analyzed. These data were obtained from a previous study (i.e., the Women, Co-occurring Disorders and Violence Study [WCDVS]) conducted by the Substance Abuse and Mental health Services Administration (SAMHSA). The terms religious coping and spiritual coping were used synonymously in this study. Their findings revealed that this population of survivors relied more frequently on positive spiritual coping compared to negative spiritual coping, and more heavily on spiritual coping overall than the general population. Negative religious coping was related to more severe trauma and mental health symptoms. More frequent childhood abuse was related to more negative spiritual coping. The size and racial diversity of the sample contribute to this study's validity, as does the adequate reliability and validity of the instrumentation used. Nevertheless, given that the study was not longitudinal in nature, causation cannot be categorically determined.

Positive religious coping also appears to have an indirect link to growth and current mood in adult survivors of childhood sexual abuse. Gall et al. (2007) examined the role of spirituality in the present adjustment of these adult survivors. A convenience sample of 101 survivors of childhood sexual abuse was recruited from a large Canadian city via advertisements displayed in universities, counseling centers, health care centers, community agencies, and newspapers. Seventeen percent of the sample were male and $82 \%$ were female, with an average age of 43 years. A large proportion of the sample (77\%) had at least some postsecondary education, and a majority identified as Christian (48\% Catholic, 24\% Protestant, $4 \%$ other Christian). Cultural background of the sample included 85\% European Canadian, 2\% Black, 3\% Arabic, and 7\% Native Canadian. Results suggest that a relationship with a benevolent God or higher power was related to 
less negative moods, a greater sense of personal growth, better resolution of the abuse, increased self-acceptance, and increased hope. While the sample did not represent high cultural diversity, it did offer evidence of the impact of positive religious coping on growth in this sample. In addition, the experience of male survivors was not well represented in this sample.

Studies on the experiences of sexual abuse survivors of diverse cultural backgrounds seem to give evidence of the value of spiritual/religious resources for overcoming, healing, and growth in the aftermath of abuse (Ahrens et al., 2010; Gall et al., 2007). For example, in a qualitative study, Smigelsky et al. (2017) examined the experiences of spiritual coping in nine Congolese refugee women who had escaped sexual violence in their home country that included rape, genital mutilation, and sexual slavery. Of the nine participants, seven had experienced sexual violence directly, while two experienced it vicariously in the person of an immediate family member (e.g., a sister). The age of the participants ranged from 24 to 66, with a mean age of 42 years. The women, who identified as Protestant Christian $(N=8)$ and Catholic $(N=1)$, took part in a 14-item semi-structured interview and consensual qualitative research methods were used to analyze the data. The small sample size and the fact that two of the women had experienced vicarious trauma requires that caution be employed when attempting to apply these findings to similar groups. Nevertheless, Smigelsky et al. (2017) found that religion/spirituality was a fundamental aspect of coping and survival in this group of survivors. 


\section{Posttraumatic Growth Among Female Survivors of Sexual Abuse}

While numerous studies have confirmed the link between the experience of sexual abuse/assault and adverse psychological sequelae in women (Campbell et al., 2009; Kilpatrick et al., 1997; Tjaden, \& Thoennes, 2006), the potential for growth in the aftermath of trauma has been a topic of inquiry in more recent years. Potential areas of positive gain have been identified by researchers and often include increased personal strength, spiritual growth/awakening, increased life satisfaction, adaptive coping, and meaning making/benefit finding. Theories of PTG have posited that cognitive processing of fearful events, frequently in the form of self-disclosure to supportive others, has been instrumental in increasing beneficial outcomes (Cann, Calhoun, Tedeschi, \& Solomon, 2010; Tedeschi \& Calhoun, 2004).

Along this line of inquiry, Hassija, and Turchik (2016) investigated the relationship of PTG to self-disclosure and mental health treatment seeking in undergraduate women following a sexual assault. Eighty-five female respondents, with a mean age of $21.35(\mathrm{SD}=6.14)$, were recruited through an online mass testing procedure administered by the psychology department at a western state university. One hundred fourteen participants were selected based on their report of prior exposure to sexual assault. Of those selected, 89 agreed to participate, representing a 78.07\% response rate. Four of those who responded were males and were excluded from the data due to inadequate sample size. Racial background was identified as Caucasian by $95 \%(n=81)$, while four respondents (5\%) lacked data on race. Seventy eight point eight percent reported being non-Latino, $80 \%$ were single, $12.9 \%$ reported living with their significant other, $2.4 \%$ were married, and $4.7 \%$ were divorced, separated, or widowed. This study 
found that survivors who utilized mental health treatment and sought supportive others for self-disclosure experienced greater PTG. In seeking supportive environments for disclosure, survivors pursue opportunities for cognitive processing and meaning making, through which growth may be fostered. While the sample in this study does not represent wide ethnic diversity, their outcome is consistent with previous research suggesting social support increases positive adjustment in sexual assault survivors (Borja et al., 2006; Cobb et al., 2006). Other research points to increased coping resources through access to emotional support, strong attachments, and the tendency to disclose sexual abuse in survivors (Frazier et al., 2004; Orchowski \& Gidycz, 2012). Processing the experience of abuse in a supportive relationship through self-disclosure may help survivors to integrate alternate perspectives into a modified personal narrative that better accommodates the painful experiences (Tedeschi \& Calhoun, 1996, 2004).

In addition to social support, some research has linked PTG to distress in survivors of sexual abuse, although the research is conflicting in this area. In one study, Lev-Wiesel et al. (2005) examined the variations in PTSD and PTG in young women survivors of childhood sexual abuse based on their relationship to the abuser. A random sample of 246 female university students, with a mean age of $24.96(\mathrm{SD}=4.45)$ served as a pool from which 93 survivors of childhood sexual abuse were drawn for his study. They reported following types of abuse: sexual harassment (46\%), rape (24\%), and sexual exploitation (30\%). Forty percent of the respondents reported being abused by a family member (parent $86 \%$, sibling $14 \%$ ) and $60 \%$ reported being abused by an acquaintance or a stranger. The study found that those who had been abused in their home by a family member experienced higher levels of both PTSD and PTG than those who were abused 
by a non-family member. The higher levels of PTSD in those abused by family members may be due to the heightened vulnerability of the abuse survivor as well as the loss of the expected social support generally provided by family members. The fact that intrafamilial sexual abuse survivors had higher levels of PTSD and PTG is evidence that PTSD and PTG are two separate constructs (not opposite ends of a spectrum) that can coexist, and may even be moderated by distress (i.e., as a mechanism that propels individuals to make changes as they attempt to manage emotional pain). The selection of acceptably valid and reliable instruments that have been used extensively in Hebrew contributes to the value of this study. However, as a cross-sectional study causation and directionality cannot be conclusively determined.

\section{Posttraumatic Growth and Psychological Distress}

Research examining the predictors of traumatic symptoms has frequently focused on the emotional and psychological distress that arises in the wake of tragic or distressing events. Thus, it seems contradictory to expect that distress can also be linked to growth in survivors of trauma. While a positive relationship between these two constructs may initially appear to be counterintuitive, attempts to identify the conditions under which PTG is most likely to develop have led to the discovery of a link between psychological distress and PTG (Cann et al., 2010; Grubaugh \& Resick, 2007).

Much of the current research on the relationship between psychological distress and PTG has, in fact, yielded mixed results (Chopko, 2010; Helgeson et al. (2006); Laufer \& Solomon, 2006; Tedeschi \& Calhoun, 2004b; Zoellner \& Maercker, 2006). However, there is considerable evidence suggesting that traumatic events can create an

environment for potential growth. For example, in a correlational study, Chopko (2010) 
examined the relationship between PTD and PTG in police officers who had been exposed to traumatic events while performing their duties. The researcher recruited 183 police officers from city police departments across a Midwestern state with varying department sizes (i.e., from 30 to 1800 officers). The racial/ethnic composition of the sample was 83.6\% European American, 13.1\% African American, 1.1\% Asian American, $1.1 \%$ Native American, and $0.5 \%$ other ethnicity. Sixty-nine percent of the officers were married, $6 \%$ were separated, $8.7 \%$ were divorced, 10.4 were single, and $5.5 \%$ were in a committed relationship. Experience in law enforcement ranged from 1 year to 40 years, with a mean of 12.6 years $(\mathrm{SD}=7.8)$. The mean number of months since exposure to the traumatic incident was 9.1 ( $\mathrm{SD}=18.5)$, and the time that had lapsed since the event ranged from 1 month to 123 months. While some studies have found unreliable relationships between distress and PTG (Tedeschi \& Calhoun, 2004b; Zoellner \& Maercker, 2006), this study found that PTG was positively related to psychological distress in police officers who had been exposed to traumatic events in the line of duty, and is in agreement with other such studies. Nevertheless, longitudinal studies in this area can better establish directionality and causation of the variables examined in this crosssectional research.

Helgeson et al. (2006) conducted a meta-analysis, reviewing the results of 87 cross-sectional studies in an attempt to explore the relationship between benefit finding (defined as "the positive effects that result from a traumatic event" p. 797-also known as PTG) and various mental (i.e., depression, anxiety, positive well-being, global distress, intrusive-avoidant thoughts, quality of life) and physical (i.e., physical functioning, selfrating of physical health) variables. Helgeson et al. chose only peer-reviewed articles to 
ensure methodological rigor and stronger conclusions. The researchers used the following keywords/terms in a computerized literature search of electronic databases: "positive life changes," "benefit finding," "posttraumatic growth," "stress-related growth," and their derivatives. Each article included in the meta-analysis had to contain (1) an adult population (2) the inclusion of a clear and validated measure of benefit finding (e.g., the PTGI; Tedeschi \& Calhoun, 1996), (3) a highly stressful event experienced by participants, and (4) a measure of physical or mental health. The review found that benefit finding was related to better mental health outcomes (i.e., less depression and greater positive well-being) and to the intrusive and avoidant thoughts that are often the consequence of living through a catastrophic or painful event. The researchers suggest that these intrusive thoughts are a form of cognitive engagement—an attempt to make sense of the event and thus work through its ramifications. Helgeson et al. (2006) contend that the process of cognitive engagement is a needed ingredient for the manifestation of growth, to the extent that this engagement allows the survivor to challenge, re-write, and reconstruct former life schemas to accommodate the new information.

Additional research has linked PTG to spirituality and social support, as well as distress. Cadell et al. (2003) examined PTG in a group of bereaved HIV/AIDS caregivers in Canada. One hundred seventy-four caregivers completed a confidential and voluntary questionnaire package. They ranged in age from 19 to 79 , with a mean age of 40.5 years $(\mathrm{SD}=11.4)$. The sample included 90 men $(51.7 \%), 80$ women $(46 \%)$, and four transgender persons $(2.3 \%)$. Of these, $51 \%$ were gay, lesbian, or bisexual and $54 \%$ were college or university educated. Seventy-nine percent completed the English questionnaire, while $21 \%$ completed the French questionnaire. The researchers 
hypothesized that spirituality, social support systems, and levels of distress would have an effect on personal growth after a highly stressful event. Using structural equation modeling, the researchers tested their hypothesis that greater PTG would occur in caregivers who (1) were more spiritual, (2) had more social support, and (3) had fewer symptoms of distress. They found that higher levels of spirituality and social support did in fact increase PTG, while lower levels of distress decreased PTG (Cadell et al., 2003). However, the inherent limitations of a cross-sectional design warrant caution in interpreting directionality of effects.

Models of PTG have contended that traumatic experiences challenge the personal assumptive world of the individual and produce a ruminative process that can eventually lead to the restructuring of their personal assumptive world (Calhoun \& Tedeschi, 2006; Linley \& Joseph, 2004). When intrusive rumination moves into deliberate rumination following a crisis, increased PTG outcomes are more likely (Tedeschi \& Calhoun, 2006). Thus, distress is believed to be the driving force behind both intrusive and deliberate rumination. While this cognitive processing is associated with higher levels of growth in trauma survivors, distress may persist.

In their model of PTG, Calhoun and Tedeschi (2006) suggest that PTG and distress are not mutually exclusive, and are likely to co-exist in the experience of the trauma survivor. In a cross-sectional study, Cabral (2010) found evidence of this coexistence of PTG and distress. The study examined the PTG in survivors of interpersonal violence who were experiencing various levels of distress. One hundred forty-three persons were recruited online for participation in this study. The convenience sample included 113 females (80.7\%) and 25 males (17.9\%); 100 participants identified as 
Caucasian (73.5\%), 13 as Asian (9.6\%), seven as Black (5.1\%), five as Aboriginal (3.7\%), one as Hispanic (0.7\%), and one as Middle Eastern (0.7\%). The mean age of respondents was $29.88(\mathrm{SD}=10.6)$, with an age range between 18 and 65 years. The research found that depressive symptoms were negatively associated with PTG, while trauma symptoms and hope were positively associated with growth. Although associations between PTSD severity and PTG were small, high levels of PTSD symptoms coexisted with high levels of PTG in some cases, providing evidence of two separate and independent constructs. Nevertheless, the cross-sectional design of this study limits the interpretation of directional effects.

A previously cited study by Lev-Wieselet al. (2005) found that women who were abused by a family member experienced both higher levels of traumatic distress and greater PTG than women who were abused by a stranger. In addition to providing evidence of a positive link between psychological distress and PTG, this study provides additional evidence that the two constructs are not mutually exclusive and may tend to co-occur.

Taku et al. (2008) examined the relationships between rumination, distress, and PTG in bereaved Japanese university students. The sample of 71 undergraduate students (33 males and 38 females) was drawn from a larger sample recruited from psychology classes at five private universities. Only students who reported having a traumatic bereavement were included in this study. Participants had a mean age of 19.94 years (SD $=1.15$ ) and an age range between 18 and 25 years. None were married, one was divorced, $60 \%$ were living with family, $38 \%$ were living on their own, and $1.4 \%$ were living in the dormitory. Participants reported bereavement/loss that occurred while they were in 
primary school (8.4\%), junior high school (11.3\%), high school (35.2\%), or university (45.1\%). The researchers used the PTGI (Tedeschi \& Calhoun, 1996) and the Impact of Event Scale-R (Asukai et al., 2002). They found a positive relationship between PTG and distress. This finding supports the Calhoun and Tedeschi (2006) model, which proposes that PTG and distress are distinct constructs, not opposite ends of a spectrum. Taku et al. (2008) tested three models using SEM, with variables including intrusive rumination, deliberate rumination, distress, and PTG, to determine the best fit to their data. Model 1 postulated that both intrusive and deliberate rumination soon after the event and more recently, lead to PTD or PTG. No connection between distress and PTG was assumed. Model 2 posits that only deliberate rumination (identified as self-initiated and solutionoriented thinking) leads to PTG, while intrusive rumination leads to distress. However, this model assumes that distress and PTG can co-exist. Model 3 hypothesized that intrusive rumination, characterized by persistent uncontrollable thoughts, is a natural part of grief, as well as other trauma, and is an indication that the person is struggling. Attempts to cope lead to deliberate rumination and continued distress following the event. This model assumes the coexistence of distress and PTG, with distress leading to PTG. Taku et al.'s (2008) analysis found that Model 3 furnished the best fit to the data. They additionally found that intrusive rumination leading to distress and deliberate rumination, both occurring shortly after the event, lead to increased PTG. Distress and PTG continued to coexist over extended periods of time.

Traumatic grief and loss appear to actuate the changes that lead to PTG. However, while substantial distress seems to contribute to higher levels of PTG, Yilmaz and Zara (2016) found that the relationship between distress and PTG was mediated by grief or 
trauma intensity. Using online and individual resources, Yilmaz and Zara recruited a convenience sample of 132 persons ( $65 \%$ female, $35 \%$ male) grieving the relatively recent loss (i.e., between five and 17 months prior to the study) of a first-degree relative or romantic partner. The relationship of participants with the deceased included the following: mother $(N=32)$, father $(N=65)$, sibling $(N=15)$, daughter/son $(N=8)$, spouse $(N=7)$, and romantic partner $(N=5)$. The cause of traumatic loss was reported as unexpected medical conditions $(N=88)$, traffic accidents $(N=27)$, other type of accident $(N=4)$, suicide $(N=5)$, death by another person $(N=3)$, and other $(N=5)$. Participants mean age was $37.43(\mathrm{SD}=11.75)$. Yilmaz and Zara (2016) found that extremely high levels, as well as very low levels, of distress seemed to interfere with the PTG process in this sample of bereaved individuals. This research suggests that there may be a curvilinear relationship between traumatic distress and PTG. However, more studies are needed to confirm this relationship.

Karanci et al., (2012) further explored the role of distress in PTG by examining the impact of traumatic stress severity and personality traits on PTG outcomes in a sample population. This research represents one part of a larger study on traumatic events, post-traumatic stress, and PTG in a Turkish community. Participants $(\mathrm{n}=969$ adults) were recruited from a stratified random cluster community sample obtained from the Turkish Statistical Institute (TURKSTAT) in Ankara (the capital city), Erzincan (a region destroyed by earthquakes in 1999), and Kocaeli (a region affected by an earthquake in 1991). Of the participants, $63 \%$ were female and $37 \%$ were male; $69 \%$ were married, $18.3 \%$ were single, $9.3 \%$ were widowed, and $2.4 \%$ were divorced. In this sample, $11 \%$ reported having experienced a traumatic accident, $28.1 \%$ had lived through 
a natural disaster, and $40.6 \%$ reported an experience of traumatic loss, illness, or death. Karanci et al. investigated the differences in PTG between accident survivors, natural disaster survivors, and individuals who had experienced the abrupt loss of a loved one. They found that the personality traits of conscientiousness, agreeableness, and openness to experience had robust predictive value for overall PTG. In addition, the study revealed that individuals with extraversion as a personality trait experienced higher levels of growth when the levels of traumatic stress were higher. Thus, in this study, extraversion appeared to have a protective effect in conditions of extreme psychological distress. Karanci et al. suggest that this may be due to the distinctive coping strategies of extroverts, which often include connecting, support seeking, verbal processing, and problem solving. Strategies such as these, which require persistent cognitive engagement, appear to be favorable to PTG.

\section{Psychological Distress: An Overview}

If the term psychological distress seems nebulous to some readers, it is likely due to the broad use of the term in depicting a wide variety of symptoms that appear either in combination or separately, and have been used extensively as markers of mental health and psychiatric disorders, as well as in the literature (Drapeau et al., 2012). Thus, the term frequently appears as an indistinctly defined concept that is used interchangeably with terms such as stress, strain, and (emotional/psychological) pain (Phillips, 2009; Ridner, 2004).

Mirowsky and Ross (2002) (as cited in Drapeau et al., 2012) defined psychological distress as the emotional pain that is manifested as symptoms of depression and/or anxiety. This definition has been widely adopted. Psychological distress has been 
known to cause mild to severe disruptions in the activities of daily living for many individuals (Wheaton, 2007). It may be activated by a stressful event and, in the absence of adequate coping resources, can lead to ineffective or maladaptive coping patterns (Horwitz, 2007). Some have argued that psychological distress occurs only in the presence of stressors and disappears when stressors are removed or when individuals learn to cope/adapt adequately (Horwitz, 2007; Ridner, 2004), making it a transient experience. Others have asserted that distress does indeed occur in the absence of stressors and is often a stable, long-term feature for many adults (Wheaton, 2007). Excessive distress (i.e., above what is culturally and developmentally appropriate) that persists far beyond the presence of the stressor is considered by many to be a sign of a mental disorder, and some researchers suggest that it must be distinguished from reasonable or "normal" responses to stressful stimuli, to avoid over-pathologizing persons (Horwitz, 2007; Phillips, 2009).

Persistent psychological distress, particularly the type that causes severe impairment to daily functioning, is an essential benchmark in the diagnosis of various psychiatric disorders. Thus, it may contribute to a diagnosis when it appears alongside the other symptoms necessary to meet criteria for that diagnosis (Phillips, 2009). Hence, it is suggested that psychological distress may be transient or persistent, may range from mild to severe, and may cause varying levels of impairment in functioning.

Over time, various approaches to mental illness and mental health have identified psychological distress according to differing interpretations of what constitutes a mental disorder. For example, the disease model attributes mental illness to physiological and chemical alterations in the brain (and elsewhere in the body) that result in harm to the 
individual (Tyrer \& Steinberg, 1998 as cited in Ryrie \& Norman, 2013). Thus, distress that contributes to mental disorder is the result of observable physiological modifications in the brain that are linked to changes in behavior. Compared to the disease model, the psychodynamic model views psychological distress as the result of a conflict between the different levels of human consciousness, in particular between the conscious and unconscious mind. This model assumes that behavior is motivated by mental activity that occurs beyond the conscious awareness. The behaviorist views distress as a symptom resulting from maladaptive responses to stressors. It is the maladaptive behavior that is regarded as the disorder, and treatment focuses on replacing maladaptive behavior patterns with adaptive ones. In contrast to the behavioral and disease models, cognitive models posit that behavior is determined by the individual's interpretation of the events. Thus, distress is the result of errors in thinking and treatment focuses on remediating dysfunctional or irrational thought patterns. Social models seek to understand the interaction of environmental influences upon individual mental health. From this perspective, distress is a mental disturbance to which all are susceptible when unpleasant and unexpected life events take place (Ryrie \& Norman, 2013).

Several lifespan, development models (Bronfenbrenner, 1992; Vygotsky, 1930 as cited in Davies, 2018) may be somewhat more conducive to the cognitive processing and restructuring of life narratives that presumably encourage PTG (Calhoun \& Tedeschi, 2006). In these approaches, distress is often framed as a common experience or life stage that presents challenges to be overcome, rather than symptoms of mental illness or a mental disorder. Thus, change and integration become normalized as necessary aspects of the life journey. 
The psychological discomfort or unpleasant emotions that sometimes develop in the aftermath of traumatic events can interfere with daily functioning and result in negative views of self, others, and the environment. While distress is experienced internally (e.g., anxiety, low mood), it may include emotional (e.g., tearfulness, irritability, angry outbursts), behavioral (e.g., self-injury, social withdrawal, poor selfcare) and physical (e.g., difficulty breathing, excessive perspiration) manifestations. Extreme levels of psychological distress lie at the core of many mental disorders. This study will examine the direct relationship of distress to PTG as well as when survivors access spiritual resources as a way to cope with disturbances.

While some research points to a correlation between PTG and distress (Cann, 2010; Grubaugh \& Resick, 2007; Lev-Wiesel et al., 2005), other studies have yielded mixed results (Helgeson et al., 2006; Laufer \& Solomon, 2006). Nevertheless, Tedeschi and Calhoun (2004a) contend that the power behind potential growth is in the severity of the trauma experienced by survivors. In this theory, psychological distress becomes a necessary ingredient in the development of PTG. They assert that it is the capacity of the event to create disorder and disruption, which then drives many survivors to engage the cognitive processes necessary for the re-assessment of major life schemas and assumptions as they attempt to make sense of the traumatic event(s). PTG is the positive change that comes about as a result of this engagement.

Research by Tedeschi and Calhoun $(1996,2004 a)$ suggests that positive personal changes occur at a greater frequency in individuals who report higher levels of traumatic distress compared to those who do not report trauma. Meaningful changes are deemed to 
be the result of the engagement of cognitive processes in the struggle to make sense of frightful events.

In survivors of trauma, distress creates an impetus toward relief seeking, frequently moving into automatic intrusive rumination. As noted previously, meaning making appears to be an essential component of readjustment and growth after trauma (Valencia et al., 2013), driven by distress. Negative core beliefs such as the shame, guilt, and self-criticism experienced by victims become part of these survivors' developing assumptions about the world and are used as a lens through which they interpret their environment, their relationships to others, and their identities. Learning to see their beliefs about themselves and the world from a new perspective and making adjustments in personal schemas in order to accommodate the conflicting traumatic experiences leads to enhanced PTG (Grad \& Zeligman, 2017; Neimeyer et al., 2006).

\section{Psychological Distress and Spiritual/Religious Coping}

A regression analysis by Pargament et al. (2000) demonstrated that religious coping is associated with variances in emotional distress, mental health, physical health, and PTG. They found that, like coping in, religious coping practices appeared in various forms. Individuals who experienced spiritual discontent and punishing God reappraisals were more likely to have poorer adjustment outcomes than those who practiced benevolent religious appraisals, religious forgiveness, and sought religious support. Consistent with this finding, a longitudinal study by Burke et al. (2011) found that a group of 46 African American homicide survivors were more likely to develop

complicated grief after a loss when they engaged in negative religious coping (e.g., anger toward God and/or feeling spiritually abandoned) than those who practiced positive 
religious coping (e.g., benevolent God appraisals and/or seeking a stronger connection with God). Participants were administered two assessments six months apart. This sample of homicidally bereaved adults relied heavily on both positive and negative religious coping as they struggled to manage their loss. However, in this study, while negative religious coping was a robust predictor of complicated grief, positive religious coping did not appear to affect adjustment significantly. The authors suggest that the psychological benefits of positive religious coping may have been concealed in this sample due to their general uniformity in endorsing high levels of positive religious coping at both assessment times.

In a seminal study by Koenig et al. (1998) negative and positive types of religious coping were identified and compared with nonreligious coping styles in 577 hospitalized senior patients. They found that negative religious coping was associated with poorer physical health, lower quality of life, greater depression, punishing God reappraisals, and expressions of spiritual discontent. Individuals who excluded God in their coping approaches or who expressed negative perspectives of God, church members, or clergy experienced higher levels of depression and lower quality of life. Religious coping behaviors had a greater correlation with mental health than even non-religious coping behaviors.

In a two-year longitudinal study, Pargament et al. (2004) examined the impact of spiritual coping on the psychological, physical, and spiritual outcomes of 268 elderly medical patients, with similar outcomes. Positive religious coping practices such as seeking spiritual support/connection, religious forgiveness, and benevolent reappraisals were predictive of better mental, spiritual, and physical health. Conversely, negative 
religious coping practices (e.g., spiritual discontent, punishing reappraisals, and demonic reappraisals) predicted declines in overall health.

Research continues to provide evidence of the relationship between spiritual coping and mental health. In a systematic review of the published research between 1990 and 2010, Bonelli and Koenig (2013) found that a majority of the studies (72.1\%) endorsed a positive relationship between religious/spiritual involvement and mental health. A smaller percentage (18.6\%) reported mixed results, while $4.7 \%$ of the studies found increased mental disorder with greater spiritual involvement. Thus, Bonelli and Koenig concluded that there is good evidence for the contention that a positive relationship exists between religious involvement and mental health.

Additional studies have suggested that negative religious coping and spiritual/religious struggle is associated with higher levels of psychological distress. Chan and Rhodes (2013) examined the relationship between positive/negative religious coping and posttraumatic stress, psychological distress, and PTG in a sample of low-income mothers and survivors of hurricane Katrina. They found that negative religious coping predicted psychological distress while positive religious coping predicted PTG. Findings by Burke et al. (2011) suggest that complicated grief was significantly related to higher levels of spiritual/religious struggle. In another study, Ramirez, et al. (2012) found that religious struggle was associated with increased depression and anxiety symptoms and decreased physical, mental, and social quality of life in patients with end-stage renal disease. Positive religious/spiritual coping predicted better mental health and social relationships. 
In survivors of sexual trauma, religious/spiritual coping has been shown to predict stress-related growth and PTG in numerous studies. For example, in a study by Gall (2006), spiritual coping tended to predict the distress levels of adult survivors of childhood sexual abuse. Gall observed that negative forms of spiritual coping (e.g., spiritual discontent) were related to higher levels of distress and depressed mood, while positive forms of spiritual coping (e.g., seeking spiritual support) were linked to lower levels of anxiety and negative mood. In a later follow-up study of 101 survivors of childhood sexual abuse, Gall et al. (2007) observed that survivors who claimed to have a relationship with a benevolent God fared better in the areas of abuse resolution, personal growth, self-acceptance, and hope than survivors who did not make that claim. In addition, survivors with positive God appraisals experienced less negative moods. In a synthesis of the literature examining the changes in personal spirituality after childhood sexual abuse, Walker et al. (2009) reviewed 34 studies. Of these, 14 studies demonstrated a decline in religiousness/spirituality and 12 indicated a combination of growth and decline. However, Walker et al. found seven studies offering preliminary evidence that religiousness/spirituality can, in some cases, moderate post trauma symptoms in survivors of childhood abuse. For example, one study (Elliot, 1994 as cited in Walker et al., 2009) compared the current functioning of abuse survivors of atheist/agnostic, conservative Christian, and other faith traditions and found that individuals who reported no religious involvement experienced higher levels of trauma symptoms than those who were engaged in some form of organized religion, regardless of affiliation. 
Some studies seem to provide conflicting evidence regarding the impact of spiritual/religious coping on potentially traumatic experiences. Bryant-Davis et al. (2011) investigated the relationship between social support, religious coping, and post-trauma symptoms using confirmatory factor analysis and structural equation modeling in a study of 413 African American female survivors of sexual assault. Their findings suggested that depression and PTSD were less likely to occur in survivors with greater social support and more likely to occur in women who practiced religious coping. However, to determine religious coping, the authors of this study selected two items from the Brief COPE (Carver, 1997), a 28-item, self-report measure used to assess various types of coping strategies. The items selected composed the religion scale of the measure (i.e., I tried to find comfort in my religion or spiritual beliefs; I prayed or meditated) and identified participants' use of religion in coping. What this scale does not identify are the types of religious coping being utilized by the participants, whether negative or positive styles of coping. Thus, it is difficult to draw specific conclusions regarding the meaning of the outcome in relationship to positive and/or negative styles of religious coping.

\section{Psychological Distress and Spirituality}

In the aftermath of painful experiences, survivors struggle to make sense of events and often reevaluate their fundamental expectations of the world. Personal beliefs, assumptions/ expectations of others, how and why things happen, ideas about purpose, and hope for the future are often acutely impacted by tragic events (Janoff-Bulman, 2004; Matthews \& Marwit, 2003; Pargament et al., 2005). When existing worldviews include spiritual/religious beliefs, there is frequently a struggle to make sense of the traumatic experience in the current spiritual framework. However, assumptions about the world 
may be shaken, shattered, or disoriented in the wake of a painful event, causing a destabilization of foundational assumptions and leading many survivors to challenge their held beliefs. This process can lead to either loss or growth of spirituality (Tedeschi \& Calhoun, 2006). For example, these challenges to spiritual meaning have been observed in parents who are grieving the loss of a young child (2006), particularly in dealing with the conflict that arises in the face of the anomalous loss of a young life.

In a case study, Ganzevoort (2001) used a narrative approach to explore the function of religion and religious changes in the lived experience of one survivor of clergy sexual abuse. Using Pargament's (1997) definition, Ganzevoort described religious coping as a search for significance that involves spiritual/religious themes. Pargament (1997) conceptualized the function of religion as both a pathway to the sacred (i.e., a means) and a destination (i.e., an end). It is suggested that both the means and the end can function to either conserve or transform held beliefs (1997). Ganzevoort explored the role of religion in the narratives of the subject at various periods in his life. He found that religious themes played differing roles at different times during the subject's history. For example, as a youth the subject developed a narrative of serving, which provided him with a meaningful counter story (p. 57) and allowed him to maintain his religious traditions while likening his sufferings to that of St. Sebastian and Jesus. This story line offered this survivor a sense of being specially selected. As he matured and discovered that his narrative was an obstacle to his relational development, he discovered new religious themes with which to build his story. In this case study, the subject encountered the challenge of conservation versus transformation of his spirituality in his attempt to understand his experience. 


\section{Psychological Distress Among Female Survivors of Sexual Abuse}

The U.S. Department of Justice (2006) has estimated that $17.6 \%$ of women have been victims of sexual violence at some point in their lives. This estimate does not include female children, adolescents, homeless or institutionalized persons. Research shows that crimes of sexual violence/rape are frequently underreported (U.S. Department of Justice, October 2018). According to the U.S. Department of Justice, just 33\% of females victimized after age 18 had received counseling to address emotional and psychological distress. Nineteen percent reported having lost time from work due to their victimization. Lost work time may be related to court hearings, counseling, medical treatment, elevated distress, depression, among other possibilities.

The cost in mental health to survivors of sexual abuse/violence/assault has been well documented (Cantón-Cortés et al., 2016; Hillberg et al., 2011; Rahm et al., 2013). For example, among female military veterans, Luterek et al. (2011) found that there was a greater likelihood of PTSD and a higher risk of exposure to additional traumatic events in survivors of sexual assault. These veterans were more likely to endorse symptoms associated with psychological distress, such as emotional dysregulation, dissociation, somatization, and difficulties with interpersonal relationships.

\section{Psychological Distress Among Female Survivors of Sexual Abuse by Religious Leaders}

Quantitative research designs rely on empirical definitions and a theoretical structure upon which to build an understanding of various phenomena. Bilsky (2013) sought to provide such a theoretical framework using a grounded study to define abuse by religious authority. A convenience sample of 15 former members of various Christian 
groups were asked to identify the factors they believed constitute abuse by religious authority. Respondents who (1) had an experience of emotional and spiritual distress that occurred in a religious or spiritual organization, (2) had perceived trauma as a result of this distress, which was perceived as being related to the authoritarian leadership, and (3) were at least 18 years of age or older, were included in this study. Persons who were not been members of a Christian group or were not fluent English speakers, were excluded. Twenty-nine individuals were part of the initial telephone interview. For various reasons (e.g., not meeting inclusion, not returning informed consent, withdrawal prior to data collection) a number of respondents were excluded and final data was collected on a sample of 15 participants (nine females, five males, one no response). The mean age of this group was $45(\mathrm{SD}=12), 53.3 \%(N=8)$ were married or partnered, $33 \%(N=5)$ were single, one was cohabiting, and one was divorced. Racial/ethnic identification was primarily Caucasian $(93.3 \% ; N=14)$, with one African American. Eight (53.3\%) of the respondents identified as currently Christian, while seven (46.6\%) identified as agnostic, atheist, or not religious. Reflecting on their own experiences of abuse, several themes emerged from the interviews. This group identified the following primary distinguishing factors: excessive authority, misuse of power, and inappropriate use of God. All of these factors were interrelated. Behaviors that were facilitated by these primary factors, such as the abuse itself, were identified as secondary factors. Hence the abuse occurred in conditions of excessive authority, misuse of power, and an inappropriate use of God. Bilsky concluded that the definition of this abuse must include both the abusive behaviors and the conditions under which they occurred. Thus, the definition of abuse by religious authority is stated as the wrongful use of the power acquired through a position of 
authority in a religious organizational body and a perceived connection with the divine (i.e., God), to exploit, harm, control or shame a less powerful individual (Bilsky, 2013). This definition was consistent with previous literature on abuse by religious leaders (e.g., Cudmore, 2002; Dehan \& Levi, 2009; Gubi \& Jacobs, 2009; Rauch, 2009).

\section{Coping Mechanisms Used by Female Survivors of Sexual Abuse by Religious}

\section{Leaders}

There is evidence to suggest that religion provides some survivors with a powerful incentive to make changes based on a desire to make sense of their own tragedy and unanswered questions (Ganzevoort, 2001). Other research points to religion as a source of strength associated with well-being in women who have been sexually assaulted (Kennedy et al., 1998), as a source of sanity decreasing susceptibility to traumatic stress after trauma (Chen \& Koenig, 2006; Israel-Cohen et al., 2016), and as a general positive correlate with recovery (Linley \& Joseph, 2004; Shaw et al., 2005) for many survivors.

However, survivors of SARL face crucial challenges. Similar to most sexual abuse survivors, they often struggle to manage the emotional and psychological distress associated with the disturbing critical event(s). In addition, female survivors of SARL are likely to (1) be (or have been) part of a faith community, (2) have encountered spiritual struggles because of their experience with abuse, and (3) have increased distress because of the relational dynamics with the abusive leader(s) and their faith community, postdisclosure. Survivors of SARL frequently find themselves unable to reconcile their experience with elements of their faith or worldview, leading to disruptions and questioning of existing life narratives. In the aftermath, survivors repeatedly review their 
distressing experiences to make sense of it and reduce discomfort. Tedeschi and Calhoun (2006) refer to this process as cognitive engagement or rumination. During this struggle, survivors who are attempting to understand their trauma may choose to adjust and exchange previously held life schemas for narratives that better fit their experiences. Many survivors of SARL choose to let go of previous spiritual/religious involvement entirely (Doehring, 1993; Falsetti et al., 2003).

Ganje-Fling et al. (2000) compared two groups receiving psychotherapy on various aspects of spiritual functioning. The goal of the study was (1) to explore how the psychological concerns of two groups (i.e., individuals seeking therapy for childhood sexual abuse and individuals seeking therapy for general psychosocial concerns) affect spiritual functioning, and (2) to assess whether the two groups differed in spiritual wellbeing from samples of hospice workers and medical patients. The first group $(N=43)$ received psychotherapy for childhood sexual abuse for an average of 50.4 psychotherapy sessions, while the second group $(N=34)$ received psychotherapy for reasons other than childhood sexual abuse for an average of 55.6 sessions. Participants were recruited from a Midwestern umbrella counseling agency, which addresses general psychosocial concerns, and includes a community agency that focuses specifically on the treatment of sexual trauma. All clients from this agency received a research packet that included a description of the study, informed consent, research instruments, and a return envelope. Of those receiving therapy for childhood sexual abuse, 43 (36 women and seven men) of the 53 returned packets were usable. Thirty-four ( 30 women and four men) of the 41 individuals receiving psychotherapy for general psychosocial concerns returned packets that were usable. Both groups had similar demographics, were predominantly White 
( $86 \%$ and $97 \%$, respectively), middle to lower income, and had a mean age of 33 years. Ganje-Fling, et al. (2000) found that individuals seeking therapy for childhood sexual abuse and individuals seeking therapy for general psychosocial concerns did not differ significantly in measures of spiritual well-being. However, both groups fared less well on measures of spiritual well-being than samples of medical outpatients and hospice workers, suggesting a possible spiritual struggle among individuals seeking therapy for both childhood sexual abuse and general psychosocial concerns.

When sexual abuse occurs in a religious context, such as at the hands of a religious leader, alienation and deep spiritual pain is often the result (Pargament et al., 2008; Walker et al., 2009). Some victims will avoid seeking help from their religious institutions if they perceive that God did not listen to their pleas for help or is in some way responsible for the violation (Franz, 2002). Victims with severe trauma and complex PTSD are more likely to view God as exacting and wrathful, or simply absent (Doehring, 1993).

Graves (2015) used a mixed methods approach in a review of archived litigation reports of adult plaintiff-survivors of childhood SARL and found some evidence of spiritual struggle. The purpose of the study was to shed light on the lived experiences of the survivors. Graves requested permission from the plaintiffs to access the legal documents necessary for this study via the law firm Kiesel, Boucher, and Larson. The firm mailed approximately 350 packets to potential participants. Each packet contained a cover letter from the attorney and the researcher, an explanation of the research, a consent form, and a return envelope. Forty-seven plaintiffs (36 males, 11 females) returned signed consent forms to the attorneys. Text obtained from the legal documents was 
treated as "proxy for experience" (Graves, 2015, p. 50) and thus analyzed qualitatively using Applied Thematic Analysis. Aspects of the documents that were quantifiable (e.g., frequency and type of abuse) were treated as quantitative data. Participants ranged in age from 27 to 68 years. Emerging themes were categorized according to (a) circumstances prior to the onset of abuse, (b) actual abuse-related events, and (c) disclosure/injuries following the onset of abuse. Prior to the onset of abuse, survivors revealed conditions that included devoutly religious families, reverence and awe of clerical leaders, befriending of family by cleric, increased attention toward the victim, and gifts of inducement. Themes that arose following the onset of abuse included spiritual disengagement, fear of disclosure, delayed disclosure, and long-term effects (e.g., symptoms of trauma, depression, substance abuse, grief/loss, spiritual struggle, anxiety, health problems, sexual and relational problems).

The psychological distress experienced by survivors is often deepened by the inadequate response of religious leaders and communities after disclosure. For example, Feit (2013) examined the manner in which the Orthodox Jewish community responded to reports of sexual misconduct in their midst, while meticulously endorsing and upholding traditional moral codes of conduct. She observed that there had been a "deafening silence" (p. 154) from the very leaders whose responsibility it was to defend Orthodox Jewish values. Feit pointed out the contradictions of a culture in which strict religious obedience, as pertains to modesty and sexual behavior, is highly regarded while sexual misconduct appears to be overlooked and even purposely ignored. She referred to the dissonance between the existing sexual misconduct and the lack of dialogue around the topic as a systemic dissociative process, resulting from the inability of the Orthodox 
Jewish community to address the existing problem. This, she contends, provides a culture that enables, denies, and minimizes sexual misconduct. Feit also noted that the intense focus on rules of dress and modesty has caused many to emphasize the law while forgetting the intention of the law (i.e., to curtail inappropriate sexual activity). Thus, appearances will often take precedence over a truth that may lead to a scandal or humiliation for a family, school, or community. Consequently, family members and community leaders may attempt to avoid the disgrace associated with sexual sin and choose to remain silent. Similarly, the religious community is fearful of being disgraced publicly if information of sexual impropriety becomes public knowledge through a lawsuit. Feit argues that the relative social isolation in this community has contributed to the belief that sexual lust is triggered/inflamed by the outside world, thus providing the Orthodox Jewish community, as well as other religious communities, with another reason to deny the existence of sexual misconduct among them.

Psychological distress that arises in the aftermath and as a result of SARL has the potential to deprive SARL survivors of a powerful coping resource. Research has identified a fairly consistent connection between spirituality/religiousness and psychological well-being (Buzdar et al., 2015; Gall, 2000; Henderson \& Ellison, 2015; Kopacz et al., 2016; Levin, 2012; Levin, 2014; Ronneberg et al., 2016). More significantly, a growing body of literature has specifically examined spiritual coping practices and its connection to PTG (Shaw et al., 2005). In one example, Ai et al. (2013) explored PTG and its relationship to religious factors in the recovery of cardiac patients. This study utilized data previously collected for a medical preoperative study. Participants were recruited from the University of Michigan Medical Center and were (1) 
at least 35 years old, (2) scheduled for non-emergency, non-transplant surgery, (3) able to speak and understand English, and (4) physically and cognitively able to provide informed consent. A sample of 262 patients who had undergone cardiac surgery, were surveyed with the goal of exploring the potential effects of preoperative religious coping on long-term PTG outcomes. Two weeks prior to the surgery, trained research assistants interviewed participants and obtained demographic information, religious factors, medical information, and mental health symptoms. A second interview was conducted two days prior to the surgery to assess positive and negative religious coping, as well as potential protective psychosocial factors, such as hope and perceived social support. This study was based on a follow-up survey that was administered 30 months after surgery. Of the 429 participants who completed the second interview, 262 completed and returned the mailed surveys. Using hierarchical regression analysis to examine a variety of religious factors, Ai et al. (2013) found that preoperative positive religious coping was predictive of PTG thirty months after surgery. They proposed that this growth could be related to the meaning-making role of positive religious coping practices.

Spiritual coping has also been positively linked to PTG in burn recovery victims (Askay, \& Magyar-Russell, 2009), victims of natural disasters (Wlodarczyk et al., 2016), marital adjustment in infertile couples (Ghafouri et al., 2016), childhood victimization (Schaefer et al., 2018), and young adults experiencing parental divorce (Milam \& Schmidt, 2018), as well as other stressful life circumstances (Chan \& Rhodes, 2013; Prati \& Pietrantoni, 2009; Vanhooren et al., 2018; Wilson et al., 2016). 


\section{Spiritual Coping: An Overview}

An attempt to measure spiritual coping would first require a definition of spirituality and an examination of whether spirituality and religiousness are decidedly separate constructs. As previously stated, this study will define spirituality as "a search for the sacred," (Pargament, 1999, p. 12), in which the term sacred refers to concepts of God, transcendence, and objects associated with this conception.

In recent years, definitions of spirituality have become increasingly distinct from concepts of religion and religiousness. Features previously identified with religion have become more closely associated with the construct of spirituality, while religion has become more narrowly defined. Pargament (1999) noted that references to religion have historically included ideas of personal and relational experiences with transcendence, as well as the institutional elements related to religious practice. However, the term religion is now more closely identified with the more limited institutional elements of religious experience (Zinnbauer et al., 1999), while the term spirituality often represents the individual experiences with transcendence and meaning (Hill et al., 2000). Daly (2005) defined religiousness as an outward (i.e., extrinsic) manifestation of "devotion or worship" (p. 1238) that comprises customs, ceremonies, and/or traditions related to the sacred. He defined spirituality as the individual's inner (i.e., intrinsic) experience to and with the sacred. In spite of the widening distinction made between these two terms, Hill et al. (2000) have found that definitions for spirituality are far from consistent in the literature (Gall et al., 2011).

In an earlier study, Zinnbauer et al. (1997) explored the differentiation that is frequently made between spirituality and religiousness. They found that religiousness 
was associated with authoritarianism, religious orthodoxy, intrinsic religiousness, selfrighteousness, and church attendance; while spirituality was associated with mystical experiences, new age beliefs and practices, and/or having a personal relationship with God or a Higher Power. However, they also found that self-rated spirituality and religiousness were still significantly correlated and almost three quarters of the respondents (74\%) rated themselves as both religious and spiritual. Both religiousness and spirituality had a similar conceptualization of the sacred, and were associated with intrinsic religiosity, frequency of prayer, and church attendance. Almost one fifth of respondents identified themselves as only spiritual and were more likely to (1) have a negative evaluation of religiousness, (2) have different ideas about what constitutes sacredness, (3) become involved in group spiritual experiences, and (4) hold nontraditional "new-age" beliefs or be agnostic. This group was less likely to engage in conventional approaches to worship.

In a phenomenological study exploring individual perceptions of spirituality and religiousness, Gall et al. (2011) asked a total sample of 234 individuals to (1) identify their degree of religiousness and spirituality on two separate 5-point Likert scales, and (2) indicate whether they perceived themselves as spiritual and religious, spiritual but not religious, religious but not spiritual, or neither spiritual or religious. Participants additionally reported on the frequency of their religious service attendance and personal prayer. Respondents were then asked (1) for their own definition of religiousness and spirituality, (2) to describe an event in which spirituality helped them to cope, and (3) to describe and an event in which spirituality interfered with their ability to cope. Participants were recruited online through Google Ads and surveys were completed via 
an internet research website. Additional printed surveys were left at several parishes in the French province of Quebec. Twenty-five participants completed the printed surveys. Participants included 43 French Canadians, 32 English Canadians, 131 Americans, 14 Europeans (including UK), and 14 others (Asia, Australia, New Zealand, Africa, and Israel). Gall et al. (2011) discovered three main themes in the definition of spirituality: (1) it was an essential feature in how the self relates to the world and others, (2) it was a guide for living and interacting with the world, and (3) it was a connection to the divine (or transcendence). In this study, the following theme emerged in relation to religiousness: Religion as a framework for personal spirituality (i.e., spirituality as the outgrowth of such religious practices as prayer and exploration of the core self). Hill et al. (2000) and others (Marler \& Hadaway, 2002) emphasize the significant overlap between the two constructs and warn against perceiving them as mutually exclusive, incompatible, or separate.

Given this overlap in practical application, spiritual coping and religious coping are viewed as interchangeable terms for this study, as they will refer to accepted religious/spiritual practices used to confront and wrestle with highly stressful life experiences. Thus, whether one identifies as spiritual, religious, or both, these coping strategies are likely to look similar.

In a meta-analysis, Ano and Vasconcelles (2005) examined the research on the efficacy of religious coping practices in dealing with highly distressing life experiences in 49 pertinent studies. They found that the studies had yielded mixed results on the relationship between religious coping and psychological adjustment. However, when they examined the impact of negative versus positive religious coping patterns in these 
studies, they found that subjects who engaged positive religious coping had more positive psychological adjustment, while those who used negative religious coping had higher levels of maladaptive adjustment.

Intrigued by the process through which religious variables appear to benefit outcomes, Pargament et al. (2000) sought to shed light on the contribution of religious/spiritual coping by developing the RCOPE (Religious Coping Scale) designed to assist in clarifying the role of religious coping in the experience of individuals who engage in them.

Early measures of religiosity focused on exploring blanket indices of religiousness, such as the frequency of prayer and church attendance. Pargament (1998) however, was interested in discovering how individuals were using these religious practices to help them make sense of and cope with major life struggles. He suggested that a closer examination of the means through which religious/spiritual coping practices were benefiting individuals in framing their life circumstances might yield greater strength in predicting favorable outcomes. He believed that this would offer researchers a richer understanding of the impact of spiritual coping compared to simply measuring the presence or absence of specific spiritual/religious practices. In the development of the RCOPE, Pargament et al. (2000) focused on representing a broad range of religious coping activities that apply to the prevailing Judeo-Christian traditions of mainstream America, noting the impracticality of attempting to reflect the global spiritual coping of all religious/spiritual traditions.

Pargament et al. (2000) based their measure of religious coping on what they believe are the functions of these practices in helping individuals to make sense of, and 
deal with, life stressors. They noted that in early religious coping research there was wide disagreement regarding the most important functions of religion. However, Pargament et al. (2000) contended that religion is multifunctional, and plays a variety of diverse roles in the lives of many individuals. In the development of the RCOPE, Pargament et al. (2000) identified five key functions of religious practice: (1) seeking and finding meaning, (2) obtaining a sense of mastery and control over one's life, (3) spiritual comfort and a reduction of apprehension about living, (4) mechanisms for facilitating intimacy with a higher power and social cohesion, and (5) assistance in major life transformations. Each function can be achieved through an array of coping methods. For example, seeking/finding meaning can be achieved through redefining a distressing experience as potentially beneficial (benevolent religious reappraisal) or labeling the event as a punishment from God (punishing God reappraisal). Conversely, any method of religious coping may serve to achieve several functions.

Pargament et al. (2000) noted that religious practices might have both effective and ineffective outcomes. While religious coping practices have been identified as positive resources for survivors of highly distressing events (Pargament, 1997), negative religious coping has been linked to anxiety, depression, and numerous other psychological disorders in the literature (McConnell et al., 2006). Through factor analysis, Pargament et al. (2000) identified positive and negative patterns of religious coping practices. Coping practices that reflect a secure relationship with God, a sense of connectedness with others, and meaningful attributions to life have been identified as positive religious coping. Benevolent religious reappraisals, religious forgiveness/ purification, and seeking religious support are examples of positive religious coping and 
were related to better adjustment. In contrast, Pargament (1997) and Pargament et al. (2003) found that punishing God reappraisals, demonic reappraisals, spiritual discontent, interpersonal religious discontent, and pleading for direct intercession were related to higher levels of distress and anxiety. The latter have been identified as negative religious coping.

\section{Spiritual Coping and Posttraumatic Growth}

Individuals who turn to religious/spiritual resources to address stressful life circumstances are said to be engaging in spiritual coping. Shaw et al. (2005) examined 11 published studies that reported an association between religion, spirituality, and PTG. Their review found that: (1) religion/spirituality is often a resource and a benefit to trauma survivors, (2) experiences of trauma can produce a deeper/stronger spirituality, and (3) positive religious coping, spiritual openness, existential readiness, religious practice, and intrinsic religiosity are linked to PTG.

Ciali (2006) examined the relationship between spirituality and PTG among individuals who had experienced the loss of a loved one. Research has supported the positive link between the practice of spirituality and the mental or physical health (Cole, 2005; George et al., 2000; Thoresen, 1999) and suggests that there are valuable resources available in this practice that offer a level of resilience in the face of trauma. Ciali (2006) hypothesized that there would be a meaningful positive relationship between religiousness/spirituality and PTG in those who had experienced loss. She found that individuals who had stronger beliefs and practices had significantly greater PTG compared to those who had less-developed religious/spiritual practices. In this study, however, the relationship was only of moderate strength. Ciali (2006) suggests that the 
moderate effect might be due to the average length of time since the traumatic event $(9.7$ years) in this study. She notes the greater effect sizes in past studies, such as by Tedeschi and Calhoun (1996), in which the relationship between these variables was greater and the average length of time since the event was shorter.

In a phenomenological study, Baty (2012) helped to shed light on the extent to which spirituality contributes to the PTG of some survivors of childhood sexual abuse. The goal of the study was to discover (1) how childhood sexual abuse survivors describe their religious/spiritual experience, (2) how they defined their religious/spiritual experience in relation to the concepts of Spiritual Journey, Spiritual Connection, and Spiritual Transformation (according to the Being Delivered framework, as cited in Baty, 2012), and (3) how the survivors perceived spirituality as being a part of their PTG process. Using a sample of eight female survivors, the author used the five domains of the PTGI (Tedeschi \& Calhoun, 1996) to examine the ways in which spirituality might be experienced as a major contributor to PTG. Participants were requested to (1) use prayer/meditation on a daily basis, (2) keep a daily journal of their prayer/meditation, and (3) attend a weekly focus group for a period of four weeks. At the end of this time period, an unstructured interview and the PTGI were administered. All five domains of the PTGI were identified as categories of positive change at the conclusion of the trial. While all participants reported spiritually influenced PTG, a greater number of participants $(37 \%)$ experienced their greatest growth in the domain of Spiritual Changes (as identified by Tedeschi \& Calhoun, 1996). Fifty percent of the participants experienced their greatest spiritually influenced growth in the areas of Connection with Others and Personal Strength, while $12.5 \%$ had greater spirituality- influenced growth in Appreciation of Life. 
This study provides insight into the ways that spirituality supports PTG.

The literature demonstrates that Faith-based factors have a positive influence on long-term personal growth (Gall, 2000; Henderson \& Ellison, 2015; Kopacz et al., 2016; Levin, 2014; Levin, 2012; Proffitt et al., 2007; Ronneberg et al., 2016). PTG outcome has been evaluated in connection with spiritual/religious coping styles after critical and highly distressing life experiences. A previously cited study on the impact of religious/spiritual coping styles on long-term PTG in the aftermath of cardiac surgery hypothesized that positive religious coping would predict greater PTG outcome (Ai et al., 2013). The authors of this study additionally hypothesized that the level of spiritual connection would explain the outcomes for religious coping. In their analysis, preoperative religious coping was found to predict growth at follow-up. Positive religious coping decreased the effects of gender, age, and race. In addition, perceived spiritual support seemed to cancel the impact of religious coping, suggesting that perceived spiritual support mediates the impact of religious coping on PTG. Ai et al. (2013 suggest that meaning-making produced by a sense of higher spiritual connection (a by-product of religious coping) may explain the higher levels of PTG experienced by these patients.

A study by Bade (2000) set out to explore how specific religious coping methods are related to different types of PTG. A sample of 239 Christian adults (63\% women and $37 \%$ men) of multiple denominations was assessed on the basis of their religious coping, PTG, and evaluation of a severely distressing personal event. Of the respondents, $90 \%$ were Caucasian, $4 \%$ Native American, 3\% Hispanic, $1 \%$ African American, $1 \%$ Asian, and 1\% non-specified. Religious affiliations included Baptist (27\%), Church of Christ (16\%), Methodist (12\%), Lutheran (10\%), Nondenominational (9\%), Catholic (8\%), 
Episcopal (6\%), Presbyterian (3\%), and other/unspecified (9\%). Bade (2000) examined levels of distress as well as religious and non-religious demographics. Findings suggested that the variance on PTG was significantly predicted by both positive and, to a lesser degree, negative religious coping. Religious coping was positively associated with all five PTGI domains (Tedeschi \& Calhoun, 1996) as follows: Relating to Others, New Possibilities, Personal Strength, Spiritual Change, and Appreciation of Life. The lack of ethnic diversity in this sample may preclude generalization to more diverse populations.

\section{The Significance of Spirituality on Survivors of Sexual Abuse}

\section{by Religious Leaders}

For many individuals, religion offers a path towards finding answers to existential questions and providing meaning to life. Religion may define a person's relationship to the world and the transcendent, often guiding morality, instilling hope, and providing a sense of purpose. Religious traditions provide avenues for the expression of personal spirituality. However, assumptive worldviews can be questioned when a severely

distressing event causes dramatic psychological and emotional upheaval. For survivors of SARL, this upheaval has a unique set of conditions. Huson (2002) found that prior to the abuse, many of the women in their study held their religious leaders in high regard, considered the role of pastor/minister/ priest/cleric to be representative of God, and regarded the church as a place of safety. An inevitable deterioration in perception of the leader's spiritual role takes place after the leader nurtures an attachment with the victim, and then exploits that developing bond to gratify sexual and physical desires.

Pargament et al. (2008) reviewed the current literature to advance an understanding of spirituality and the impact of SARL on its survivors. They defined 
spirituality as a search for the sacred, which involves (1) discovery, (2) conservation or maintenance, and (3) transformation. SARL can affect the search for the sacred at each level of engagement (Pargament et al., 2008). In the discovery stage, which often takes place during childhood, SARL can stunt the natural formation of spiritual development, as the betrayal of trust hinders faith in God and religious/spiritual teachings. There is evidence that childhood SARL has far-reaching consequences in the lives of the victimized (e.g., Doyle, 2009; Easton et al., 2019; Fater \& Mullaney, 2000; Flynn, 2008; Guido, 2008; Van Wormer \& Berns, 2004).

Pargament et al. (2008) refer to the maintenance level of engagement as a process of "conservation" (p. 402) in which organized religion functions to promote the practice of spiritual activities intended to support, strengthen, and preserve the developing relationship with the sacred. Once in place, these spiritual practices and the belief systems they represent become resilient to many life stressors (2008). In the face of adversity, spiritual values are reinforced and preserved through practices that include spiritual coping methods, such as interpreting adverse events from a generous spiritual mindset, seeking the guidance of religious leaders, finding support in fellow believers, finding strength in God, releasing control, and practicing forgiveness. These and other spiritual coping practices have been associated with psychological benefits for the believer (Ano \& Vasconcelles, 2005; Pargament, 1997).

As a violation of sacred trust, SARL often presents a severe challenge to existing personal views of the sacred (Pargament et al., 2008). It is precisely because the practices involved in conservation are so effective in reinforcing spiritual values that SARL presents such a threat to the person's inner being. Victims experience this as a profaning 
or desecration of the sacred, and it can produce intense affective responses (Pargament et al., 2005).

Pargament et al. (2008) note that the experience of desecration occurred at various levels. First, the offense becomes closely linked to the sacred rituals and symbols of the system in which the violation took place. Thus, the very activities previously used to strengthen and preserve the relationship with the sacred have been violated. Second, the system that gave the offender legitimacy has also been desecrated. This is particularly distressing if the system failed to address appropriately the actions of the perpetrator, did not act to protect the victim, or acted in a manner to conceal the offense. Third, the sacred role of the spiritual leader, and the sacred relationship between the leader and the believer, has been violated. The fourth desecration occurs in the most vulnerable parts of the human identity - in the core of the soul. This personal violation can alter every aspect of the survivor's life, affecting self-concept/self-worth, interpersonal relationships, emotional regulation and expression, job performance, home life, etc. (Fater \& Mullaney, 2000). Several SARL survivors described their personal experiences in a phenomenological study:

I feel my thoughts have been invaded and my body has been invaded. I have a lot of trouble trusting people.

This guy had my soul in his hand. It was devastating to know that someone would step out of the powers of spiritual liberty to take over some else's soul... I still have anger about a lot of that and I think more of the anger is about the spiritual loss than anything to do with the sexual abuse. 
...the street life... was a way for me to beat myself over the head since the church hadn't protected me...So I thought of taking it out on others (Fater \& Mullaney, 2000, p. 289, 290).

The devastation and confusion created by SARL can destabilize spiritual footing and threaten previously firm beliefs regarding the sacred. A spiritual struggle ensues, leading the individual to transformation or disengagement (Pargament et al., 2008).

Pargament et al. (2008) identify transformation as the outcome of a struggle with spiritual questions, prompted by inner or outer tension. In the search for the sacred, transformation may occur in the character or in the significance given to the sacred in the individual's life. Pargament et al. (2008) identified three forms of spiritual struggle: (1) a struggle in relation to the divine, such as feelings of anger toward God for not protecting him/her; (2) a spiritual struggle in relation to the system of religion/spirituality, resulting in interpersonal conflict with family members, fellow church members, religious leaders, and the institution itself; and (3) an intrapsychic conflict in the form of religious uncertainty and internal discord between thoughts, feelings, and behaviors (Pargament et al., 2005). SARL survivors are likely to experience spiritual struggle in all three areas.

\section{Spiritual Coping, Psychological Distress, and Posttraumatic Growth}

\section{Among Female Survivors of Sexual Abuse}

Early studies examining the role of spiritual coping during traumatic life events have often found that religious variables are linked to more favorable mental health outcomes and can be a valuable resource for healing and growth in many individuals (e.g., Bergin et al., 1987; Koenig, 1997). Pargament et al. (2000) observed that among certain populations, religious practice was frequently cited as a source of strength and 
beneficial coping in situations of intense stress. Additional findings have linked religious involvement to decreased depression (Buzdar et al., 2015; Koenig \& Cohen, 1992; Lee, 2007), increased physical health (Harris, 1995; Sodhi \& Manju, 2014), increased mental health (Pargament et al., 1994; Henderson \& Ellison, 2015), stress-related growth (Park \& Cohen, 1993; Park et al., 2009), decreased suicide ideation in military veterans (Kopacz et al., 2016), and lower death rates (Idler et al., 2017), to name a few examples. Research by Vilencia et al. (2013) provided some insight into the power of meaning making. They found that meaning making was an essential component of readjustment and growth after trauma. They explored the pathway to growth in the posttrauma experience of two female survivors of childhood sexual abuse. The researchers found that negative core beliefs such as the shame, guilt, and self-criticism became part of their developing childhood assumptions about the world and were used as a lens through which they interpreted their environment, their relationships to others, and their identities. Meaning making became a significant part of the PTG process as the women learned to see their beliefs about themselves and the world from a new perspective, making adjustments in personal schemas to accommodate the conflicting traumatic experiences.

However, studies reveal that the experience of being sexually abused frequently had the effect of causing survivors to distance themselves from previously valued spiritual/religious practices. In cases where survivors retained spiritual involvement, traumatic symptoms were significantly diminished compared to survivors who had no religious/spiritual involvement. Walker et al. (2009) reviewed 34 studies on the changes in religion/spirituality after childhood abuse and found that a majority of survivors 
experienced either a decline (14 studies) or both decline and growth (12 studies) in spirituality/religiousness after the abuse. Seven studies found that participants who reported no current spiritual or religious affiliations experienced higher levels of traumatic symptoms than participants who were currently practicing some form of organized religion, regardless of faith tradition.

\section{Spiritual Coping, Psychological Distress and Posttraumatic Growth}

\section{Among Female Survivors of Sexual Abuse by Religious Leaders}

In times of high stress, spiritual coping resources are relied upon to help relieve emotional and psychological distress. However, a religious identity does not always serve as a protective factor in survivors of SARL. Collins et al. (2014) examined the impact of SARL on female survivors. In a qualitative study using a narrative design, they explored the role of religious experiences/beliefs in the lives of women who had been sexually abused as children. Eight Catholic-raised survivors of childhood SARL were interviewed after having received at least two years of psychotherapy. Respondents ranged in age from early thirties to mid-sixties and were diverse in sexual orientation, marital status, and ethnicity. The semi-structured interviews included the following questions: (1) Tell me about your experience of growing up Catholic, (2) How about your experience with Catholicism now? (3) Do you feel as though Catholicism played a role in the abuse you suffered? (4) What role has Catholicism played in your recovery? (5) What should a psychotherapist be aware of to work well with Catholic women survivors of childhood sexual abuse? (6) Is there anything additional that is important to you that I have not addressed? They found that the participants' religious identities had the power to either exacerbate or mitigate trauma and stress-related difficulties associated with their 
experiences. In addition, many of the women expressed that although their religion was central to their identity, its role in their recovery was rarely explored in psychotherapy.

Pargament (1997) found that a large proportion of the population obtained comfort/ support from their faith practices when faced with distressing life circumstances. For others, however, religious schemas became an obstacle to healing. It is not difficult to understand how this might be the case for victims of SARL. Survivors will often speak of their fractured relationships with God and the church after an experience of SARL (Pargament et al., 2008). While the psychological damage inflicted by tragic life events has been frequently examined in the literature, spiritual wounds are less often explored. In the context of SARL, spiritual wounds are likely to create an obstacle to psychological healing and PTG, given previous research pointing to the positive connection between spiritual involvement and well-being (e.g., Buzdar et al., 2015; Henderson \& Ellison, 2015; Kopacz et al., 2016; Levin, 2014; Ronneberg et al., 2016).

A study by Gall (2006) sheds additional light on how differing religious schemas influence the trauma and recovery outcome. Gall explored the role of spiritual coping behaviors in the lives of 101 self-identified adult survivors of sexual abuse. Respondents ranged in age from 19 to 66 with a mean age of 43.2 years and $17.8 \%$ were male. Most of the sample had post-secondary education (77.2\%), most were employed (63.4\%), and $43.6 \%$ had a family income above $\$ 40,000$. The sample was primarily Caucasian (85.1\%), with 2\% Black, 3\% Arabic, and 6.9\% Native. Participants responded to questions regarding the abuse, their coping strategies, available support systems, distress levels, and spiritual practices. Findings suggested that the spiritual coping engaged in by the participants predicted the level and type of distress they experienced. Self-directed 
behavior and active surrender were positively correlated with anxious mood, while higher levels of passive deferral predicted lower levels of anxiety. Negative forms of coping such as spiritual discontent (e.g., being angry with God) were associated with higher levels of distress, while positive forms such as spiritual support and forgiveness were related to less distress and depression. More severe forms of abuse resulted in more negative forms of spiritual coping. Thus, it was noted that, when examining the role of spirituality in the daily functioning of sexual abuse survivors, there appears to be a difference in outcome between positive and negative spiritual coping behaviors.

\section{Summary and Analysis of the Literature Review}

The intention of the present study was to examine the relationship between distress, spiritual coping, and PTG in female trauma survivors who are experiencing various levels of distress because of SARL. The goal of this review was to explore the concepts of PTG, psychological distress, and spiritual coping, in an effort to understand the relationships that exist in the literature between these variables.

PTG has been identified as a form of growth that occurs in the midst and aftermath of suffering — a notion that has existed for millennia. However, research has now provided evidence of the existence of this phenomenon (Park et al., 1996; Tedeschi \& Calhoun, 1996) which has been conceptualized in a variety of ways. Using the theoretical model of Tedeschi and Calhoun (2004a), PTG has been described as the positive changes that can develop as a consequence of being confronted with severely crippling or painful events. These critical events engender negative emotions that can lead survivors to challenge life narratives/schemas they held and eventually lead to adaptive changes in thinking and behavior. These changes must exceed pre-crisis 
functioning in at least one specific area (e.g., enhanced relationships and interpersonal behavior, a perception of new life possibilities, a new awareness of competence/capability, an awakened spirituality or change in belief system, and/or increased gratitude and valuing of life) to be considered PTG.

Tedeschi (1999) found that the positive growth identified as PTG is closely linked to the negative aftereffects of trauma - the level of psychological distress that is experienced by the survivor. Shattered assumptions about the world can leave individuals struggling to rebuild their fundamental beliefs, while they attempt to integrate the negative experience. In survivors of violence, specific cognitive processes, such as deliberate rumination (Taku et al., 2009) and meaning making (Vilencia et al., 2013), appear to increase the probability of experiencing positive change in the assumptive and interactive world, as well as in their beliefs about the future. Having a story that accommodates the trauma also appears to mitigate its negative aftereffects. The purpose of such stories is to diminish emotional distress by making the world comprehensible again. Thus, the restructuring of life narratives contributes to the meaning making process that facilitates PTG. Reported changes in self-perception and purpose precede the active changes that are evident to others, such as talking about the experience and becoming involved in efforts to prevent similar occurrences to themselves or others (Tedeschi, 1999).

For survivors of SARL, the life narrative includes religious/spiritual perspectives that shaped their stories and their identities. These perspectives provide a framework for understanding and deriving meaning and purpose. Spiritual coping practices often provide a resource for confronting difficult life circumstances and have been found to be 
related to increased psychological well-being (Buzdar et al., 2015; Levin, 2014; Ronneberg et al., 2016). However, many survivors of SARL face the conflicting experience of confronting the very system that caused them harm while simultaneously attempting to access spiritual coping resources that are associated with that system. Not surprisingly, many survivors of sexual abuse report changes (i.e., decreases) in religious/spiritual beliefs and involvement following the trauma (Falsetti et al., 2003).

Nevertheless, while some survivors of SARL may experience a decrease in spirituality or religiosity, others may reconfigure religious schemas and experience spiritual growth that exceeds previous levels. Based on research that supports a connection between positive spiritual coping and psychological adjustment in individuals faced with painful life events (Ano \& Vasconcelles, 2005; Gall et al., 2007), the current study predicted that survivors of SARL who retain their spirituality and utilize positive spiritual coping practices as ways to manage distress will also experience higher levels of PTG than survivors who report decreases in spiritual involvement and/or coping. In addition, higher levels of psychological distress were expected to predict greater PTG overall, with the highest levels of PTG expected in survivors using positive spiritual coping. 


\section{CHAPTER 3}

\section{METHODOLOGY}

\section{Introduction}

This study explored the mediating role of spiritual coping on PTG outcomes in adult female survivors of SARL who are experiencing various levels of psychological and emotional distress. The study also examined the relationship between distress and PTG as well as the impact of positive and negative spiritual coping on PTG. This provided greater insight into the nature of the relationship between distress, spiritual coping, and PTG.

This chapter discusses the research design that was chosen for this study and provides an overview of the characteristics, rationale, and limitations of the design. I next discuss the target population, the means for obtaining the sample, and the size of our sample followed by a review of the variables measured, a description of the instrumentation used, and an overview of the validity/reliability of said instruments. I conclude this section with a discussion on the sequence of the study and how the data was analyzed.

\section{Research Questions and Hypotheses}

This study examined the following questions: (1) What is the context and nature of the SARL in our sample of female survivors? (2) What are the effects of spiritual 
coping styles on PTG among female survivors of SARL? (3) What are the effects of distress on PTG among female survivors of SARL? (4) To what extent does spiritual coping mediate the relationship between distress and PTG among female survivors of SARL?

This study hypothesized that spiritual coping would mediate PTG outcomes in female survivors of SARL with high levels of distress. It was assumed that PTG and distress would co-exist. However, higher levels of distress coupled with high levels of spiritual coping were expected to predict greater PTG outcomes than high levels of distress coupled with low levels of spiritual coping. This investigation additionally explored whether positive spiritual coping practices would yield greater PTG outcomes than negative spiritual coping practices for all levels of distress. This study hypothesized that: (1) Spiritual coping would be positively associated with PTG and survivors who practice positive spiritual coping would experience higher levels of PTG than those who practice negative spiritual coping; (2) Distress would be positively associated with PTG and survivors with low levels of distress would experience less PTG than those with high levels of distress; (3) Survivors with high levels of distress and positive spiritual coping styles would experience higher levels of PTG than women with high levels of distress practicing negative spiritual coping.

\section{Research Design}

The current study is a quantitative, correlational, non-experimental design. This investigation examined the role of spiritual coping in the relationship between distress and PTG among female survivors of SARL via self-report measures and survey data. The data was collected using the secure online survey software provided by SurveyMonkey. It 
was evaluated using path analysis to explore the causal relationships between the independent variables (i.e., distress and positive/negative spiritual coping styles) and the dependent variable (i.e., PTG). Path analysis is a multiple regression methodology used prominently in the social sciences due to its suitability to examining causal relationships between a dependent variable and more than one independent variable. Using this approach, investigators can gather data in naturally occurring environments without the artificial manipulation of variables present in experimental designs. The analysis confirmed how much of the variance in PTG was explained by different levels of distress and spiritual coping styles.

Data for this study was gathered using a survey comprising three empirically validated instruments, a demographic questionnaire, and a context-of-abuse questionnaire. Self-report measures were used to collect data that cannot be observed directly and is subject to participant perception. The survey method combined ease of administration with the power to obtain extensive and understandable quantitative data. However, self-report measures offered no information about the reason participants responded the way they did, or how participants interpreted survey items, or potentially confounding variables. Some examples of variants that may affect participant responses include tiredness, time of day, and social desirability.

\section{Participants}

Requirements for inclusion in the current study were (1) female identification, (2) 18 years or older, and (3) having at least one self-identified experience of sexual abuse by a religious leader. The goal of this study was to understand the nature of the relationship between distress, religious/spiritual coping, and PTG in female survivors of SARL. 
Participants in this study were recruited from among the members of The Hope of Survivors (THOS), a U.S. national, non-profit organization providing support, assistance, and counseling to individual women who have experienced SARL. The THOS database included several thousand women who are mostly of Christian faith backgrounds. A link to the secure online survey was provided to the director of THOS. The survey link was then sent electronically to all of the members of the THOS' database. A total of 143 individuals completed the survey. Of those, 113 participants met the criteria for inclusion and made up our final sample. Informed consent was obtained from the participants and personal identifying information was maintained confidential. Demographic information was reported in the results section.

\section{Definition of Variables}

This study examined the relationship between three main variables: distress, spiritual coping styles (negative and positive), and PTG. All of the participants were selfidentified adult female survivors of SARL.

\section{Distress}

The psychological discomfort or unpleasant emotions that sometimes develop in the aftermath of traumatic events. This definition is based on The McGraw-Hill Concise Dictionary of Modern Medicine (2002), "The end result of factors-e.g., psychogenic pain, internal conflicts, and external stress - that prevent a person from self-actualization and connecting with significant others." These feelings impact/interfere with daily functioning and can cause negative views of self, others, and the environment. This study uses the terms psychological distress and distress interchangeably. 


\section{Spiritual Coping}

The use of religious and/or spiritual activities as a means of confronting, grappling with, and addressing highly stressful life occurrences (Pargament et al., 2005). This includes a variety of activities that differ according to faith traditions and cultures, such as prayer (individual, intercessory, or group prayers), meditation, practicing forgiveness, singing songs of praise/worship, spiritual re-appraisals, connecting with a community of believers (2005). Negative and positive patterns of religious coping have been identified in the literature (Pargament et al., 2000). Positive spiritual coping practices reflect a secure relationship with God, a sense of connectedness with others, and meaningful attributions to life. Negative spiritual coping practices reflect punishing God reappraisals, demonic reappraisals, spiritual discontent, interpersonal religious discontent, and pleading for direct intercession (Pargament, 1997; Pargament et al., 1998).

\section{Posttraumatic Growth}

"The positive psychological change experienced as a result of the struggle with highly challenging life circumstances" (Tedeschi \& Calhoun, 2004b) This growth may be evidenced in various ways, including increased gratitude and appreciation for life, deeper interpersonal relationships, a sense of greater inner strength, a re-adjustment of priorities, and a fuller spiritual/existential life (Tedeschi \& Calhoun, 1996, 2004b). PTG is growth that surpasses the previous functioning in one or more of these areas.

\section{Measures}

The survey for this study comprised a Demographic Data Sheet, a Context of Abuse Questionnaire, and three empirically validated instruments: (1) the Brief RCOPE (Pargament et al., 2011) which measured the use of spiritual coping styles; (2) the 
Posttraumatic Growth Inventory (PTGI; Tedeschi \& Calhoun, 1996) which measured PTG; and (3) and the Emotions Thermometer (ET; Mitchell, 2007), which measured emotional/psychological distress.

\section{Demographic Data Sheet}

Participants provided the following demographic information: age, ethnicity/race, age at which the abuse occurred, the designation of the abuser (e.g., pastor, priest, elder), family socioeconomic background, education level, relationship status, religious/spiritual preferences, level of religiosity/spirituality, and prior counseling experience.

\section{Context of Abuse Questionnaire}

Participants were asked to provide information about the context in which the abuse occurred. This section of the survey included questions relating to the age at which the abuse occurred, the number of times it occurred, the length of time it continued, the nature of the abuse, whether it occurred with more than one religious leader, the title or position of the leader(s), and whether survivors sought counseling in the two years following the abuse.

\section{The Brief RCOPE}

Participants completed the Brief RCOPE (Pargament et al., 1998), a 14-item measure that was adapted from the longer version of the RCOPE (Pargament et al., 2000). As with the original RCOPE, the Brief RCOPE was designed to capture the abundance of spiritual/religious coping practices that contribute to the faith experience, particularly in the midst of distressing life circumstances. It reflects five religious functions that include spirituality itself and the search for the sacred. These functions include (1) meaning (i.e., frameworks for interpreting life, suffering, and perplexing life 
circumstances/experiences; (2) control (i.e., avenues that help individuals achieve a sense of mastery or discipline; (3) comfort/spirituality (i.e., systems that help reduce apprehension about living in an unpredictable world); (4) intimacy/spirituality (i.e., methods of fostering social identity, support, and intimacy with members of a religious group and/or God); and (5) life transformation (i.e., a framework for finding new sources of meaning and significance). The instrument additionally provides scale items that correspond with cognitive approaches to religious coping that involve ways of thinking, acting, feeling, expressing, and connecting.

The original RCOPE is a comprehensive measure of religious coping and contains 21 subscales, each with five items. Through factor analysis, Pargament et al. (2000) identified two factors and labeled them as positive and negative religious coping. In developing the Brief RCOPE, seven items were selected from each of the two factors, representing the seven subscales of the full RCOPE. These two factors became the subscales of the Brief RCOPE.

The religious coping theory (Pargament et al., 2000) upon which this instrument was constructed posits that spiritual/religious coping can be adaptive or maladaptive. Thus, the Brief RCOPE reflects both positive and negative religious coping methods. Items that reflect a mostly secure relationship with the sacred and a sense that life is meaningful beyond the current circumstances are identified as positive religious coping (PRC), while those that reflect tension, conflict, discontent, and struggle with God and others are identified as negative religious coping (NRC). However, no assumption is made regarding the adaptive nature of negative versus positive coping methods. 
Pargament et al. (2000) contend that the efficacy of a specific coping method will be subject to the interaction of personal, contextual, social, conceptual, and cultural factors.

A literature review of the validity values for the Brief RCOPE (Pargament et al., 2011) found good predictive, concurrent, and incremental validity of PRC and NRC, as well as internal consistency (Cronbach's alpha for PRC $=0.90$; Cronbach's alpha for $\mathrm{NRC}=0.87)$. Confirmatory factor analysis of the positive and negative scales found a reasonable fit for the data and acceptable goodness of fit indices (Pargament et al., 1998). PRC had a higher mean and greater variance than NRC $(t(526)=22.42, p<.001)$ in a sample of college students (Pargament et al., 1998). In their review, Pargament et al. (2011) found that the presence of PRC predicted greater well-being, while the presence of NRC predicted poorer adjustment. Further studies found evidence for the incremental and concurrent validity of the PRC/NRC scales (e.g., Cotton et al., 2006; Van Dyke et al., 2009). In addition, the PRC and NRC were found to correspond as anticipated with other measures of mental health and well-being (Pargament et al., 1998).

The items in the Brief RCOPE are measured along a 4-point Likert Scale ranging from 1 = "not at all" to 4 = "a great deal." Scores are determined by obtaining a sum of the PRC and NRC subscales. The PRC scale includes items 1 through 7, and the NRC scale includes items 8 through 14. Higher PRC/NRC scores indicate greater use of positive or negative coping strategies, while lower scores indicate less frequent use of those strategies.

\section{Posttraumatic Growth Inventory}

The online survey completed by participants included the PTGI (Tedeschi \& Calhoun, 1996), a 21-item measure used to assess positive changes after trauma. The 
measure uses a six-point Likert-type scale, with scores ranging from $0=$ "I did not experience this change as a result of my crisis" to $5=$ " I experienced this change to a very great degree as a result of my crisis.”

The subscales for the PTGI measure five domains of potential growth and include: five items measuring New Possibilities (i.e., items 3, 7, 11, 14, and 17; e.g., "I established a new path for my life"); seven items measuring Relating to Others (i.e., items $6,8,9,15,16,20$ and 21; e.g., "I have a greater sense of closeness with others"); four items measuring Personal Strength (i.e., items 4, 10, 12, and 19; e.g., "I discovered that I am stronger than I thought I was"); two items measuring Spiritual Change (i.e., items 5 and 18; e.g., "I have a stronger religious faith"), and three items measuring Appreciation of Life (i.e., items 1, 2, and 13; e.g., "I have a greater appreciation for the value of my own life"). A total score is derived from the sum of the scores across all items. Subscale scores are obtained from the sum of the scores for the items of that domain. Higher total and/or subscale scores represent greater levels of total or domainspecific PTG, while lower scores suggest lower levels of total or domain-specific growth. The PTGI is a frequently used and well-supported instrument. The full-scale PTGI demonstrates good internal reliability with internal consistency alpha estimated at 0.90. The subscales of the PTGI have also shown good internal consistency as indicated by the following alpha levels: New Possibilities (.84), Relating to Others (.85), Personal Strength (.72), Spiritual Change (.85), and Appreciation of Life (.67). The full-scale PTGI has test-retest reliability over two months of alpha $=.71$ (Tedeschi \& Calhoun, 1996). Confirmatory factor analysis supported the five-factor model as the best fit for the data (Taku et al., 2008). 
The PTGI was developed for use with a general population of trauma survivors (Tedeschi \& Calhoun, 1996). While the PTGI was not created specifically for survivors of SARL, it has been demonstrated to be an effective of measure of growth for adult sexual assault survivors seeking treatment for trauma (Grubaugh \& Resick, 2007). In addition, it has been used and validated in numerous populations with differing levels of trauma (Tedeschi \& Calhoun, 1996). For example, it has been used effectively in studies of young adults (Calhoun et al., 2000) and adolescents (Milam et al., 2004) who have experienced trauma; with breast cancer survivors (Cordova et al., 2001); as well as with many other populations. Other studies found that PTGI scores for trauma survivors were significantly related to the subjective reports of observers connected to the survivors (Shakespeare-Finch \& Barrington, 2012; Shakespeare-Finch \& Enders, 2008).

\section{Emotion Thermometers}

Distress was measured using the Emotion Thermometers (ET) (Mitchell et al., 2012). The five-item ET utilizes visual depictions of thermometers on which participants indicate their subjective level of emotional discomfort using an 11-point scale, where $0=$ None and $10=$ Extreme. The first four items measure the participants' perceived levels of (1) distress, (2) anxiety, (3) depression, and (4) anger in the previous week. The fifth item assesses the degree to which participants feel they need help with their current discomfort/distress. It uses an 11-point scale in which $0=$ "Can manage on my own" and $10=$ "Desperately needing help." For each item, participants circle the number on the image of a thermometer that best describes their level of experience with the given emotion. Each item represents one subscale score. A total score is derived from the sum of the scores across all five items. Higher scores represent higher levels of emotional 
distress. Scores between zero and three indicate manageable levels of distress; scores between four and seven reveal moderate levels of distress; scores between eight and ten express extreme levels of distress.

Research for the ET demonstrated robust psychometric properties (Mitchell, 2007). It is believed that the ET will detect distress in survivors of SARL due to the enduring symptoms associated with this trauma. The ET was developed for use with cancer patients undergoing treatment in medical settings. It has also been used with individuals of multiple ethnic and racial backgrounds and in several different countries. The ET has demonstrated consistency with other measures of emotional distress such as the Beck Depression Inventory and the Brief Symptom Inventory. In addition, it detects clinically significant distress at 77\% sensitivity (Hughes et al., 2011; Mitchell, 2008).

\section{Procedure}

The following procedure was submitted to the Institutional Review Board along with the research proposal for consideration and approval before implementation. The current study used a quantitative, correlational, non-experimental design. The selected self-report measures, the Demographic Data Sheet, and the Context of Abuse Questionnaire were assembled into an online survey using Survey Monkey, a web-based service that manages internet surveys. The data collected by Survey Monkey was encrypted, secure, and stored centrally with Survey Monkey for ease of statistical analysis. Participants had electronic access to the survey through a secure link. The survey was designed to assess levels of PTGI (Tedeschi \& Calhoun, 1996), religious/ spiritual coping styles using the Brief RCOPE (Pargament et al., 2011), and levels of current distress using the ET (Mitchell et al., 2012) in survivors of SARL. 
The director of THOS reviewed, authorized, and emailed the survey URL link to THOS members with a request for voluntary participation. Using the provided link, participants had access to the initial page of the survey on which they were required to confirm that they were at least 18 years of age before they were permitted to continue. Next, participants connected to an informed-consent page that described the necessary details, risks, and benefits of participating in the study, including the option to discontinue participation at any time. Once participants confirmed that the study had been carefully described to them, they consented to continue with the survey. Participants connected to the demographic portion of the survey, which asked them to confirm their gender and gathered information regarding age, ethnicity, education, socioeconomic status, marital/relationship status, religious/spiritual affiliation, and level of spirituality/religiousness. This portion of the survey also asked participants to confirm that they had experienced SARL, the age at which the abuse occurred, by whom (e.g., by a pastor, priest, etc.), and the nature of the abuse. The last two questions in this section asked about the participants' experience with counseling. The response formats for the items in this section were: multiple choice, Yes/No, and one-word responses.

After completing the demographic and context portion, participants completed the PTGI, the Brief RCOPE, and the ET. If an individual participant became distressed due to her participation in this study, she was given the option of discontinuing at any time. In addition, counseling support was provided by THOS. Finally, those who completed the survey were thanked for their participation in the closing comments. 


\section{Data Analysis}

Once the desired number of responses were received, the data was obtained from the survey company. Access to the data was limited to the researcher and dissertation committee members.

The data were reviewed and analyzed using path analysis and descriptive statistics. Path analysis is a frequently used method of analysis in the social sciences that allows researchers to evaluate causal relationships between two or more independent variables and a dependent variable. For this study, a path analysis allowed the researcher to examine how levels of distress interact with positive and/or negative spiritual coping to affect the outcome of PTG. Additionally, this approach permitted the researcher to observe indirect as well as direct effects of distress and spiritual coping on PTG.

The principal goal of this analysis was to determine whether positive and/or negative spiritual coping styles contribute meaningfully to the PTG outcome in a group of women survivors of SARL, who are also experiencing varying levels of distress. The analysis also explored the direct relationships between each of the variables. This investigation first determined the strength of the bivariate correlations and constructed a model and graphical representation establishing the direction of the flow. A multiple linear regression analysis examined the extent to which PTG can be predicted by distress and positive/negative spiritual coping. The researcher then examined the model for statistical significance and the percentage of variance explained. This process is described in greater detail in Chapter 4. 


\section{Summary}

The primary goal of this study was to examine the mediatory role of positive and negative spiritual coping on distress and PTG in adult female survivors of SARL. The investigation explored whether distress had an impact on the positive/negative spiritual coping practices of survivors, and how spiritual coping might in turn impact PTG. The analysis additionally sought to explore the direct relationships between distress and PTG, and spiritual coping and PTG, as well as to understand the context and nature of the survivors' experience. High levels of distress were hypothesized to increase PTG, as was positive spiritual coping. Additionally, high levels of distress and positive spiritual coping were hypothesized to yield greater PTG than distress alone.

Data was collected from a sample of 113 participants using a secure online survey format. PTG was measured using the PTGI (Tedeschi \& Calhoun, 1996), positive and negative religious/spiritual coping were measured using the Brief RCOPE, (Pargament et al., 2011), and emotional distress was measured using the ET (Mitchell, 2007). Demographic information was collected using the Demographic Data Sheet and the Context of Abuse Questionnaire provided information regarding the nature and circumstances of the abuse. Data were analyzed using path analysis, a method suitable for non-experimental designs in natural, unmanipulated environments. All of the participants were female, 18 years or older, and identified at least one experience of sexual abuse by a religious leader. 


\section{CHAPTER 4}

\section{RESULTS}

\section{Introduction}

The purpose of this study was to explore the relationship between distress, spiritual coping, and PTG and to determine whether spiritual coping mediates the relationship between distress and PTG in female survivors of SARL. In this chapter, I present the demographic and contextual characteristics of the sample, as well as the results for each of the research questions. Demographic data, the context of abuse, levels of coping, distress, and PTG were analyzed using descriptive statistics. Reliability tests were used to examine the measures of spiritual coping, distress, and PTG. Specific relationships between distress, spiritual coping, and PTG were examined using Path Analysis. The significance level was set at .05 .

\section{Description of Sample}

The following were the requirements for inclusion in this study: (1) female identification, (2) 18 years or older, and (3) having at least one self-identified experience of sexual abuse/assault by a religious leader. A total of 143 individuals attempted the survey and met the inclusion criteria. Respondents who had $10 \%$ or more missing responses to the Brief RCOPE and the PTGI were deleted from the data set. Thus, a total of 30 questionnaires were removed from the data set as a result of having too many 
systematic missing responses to the Brief RCOPE and the PTGI, leaving a sample of 113 participants. Given that any discussion about past sexual abuse has the potential to revive memories that may lead to fear, anxiety, or depression, respondents were advised that they had the option to discontinue the survey at any time in the event that a survey item might generate an intense reaction. Respondents were additionally provided with contact information for support services through THOS. All of the participants were members of THOS at the time of the study $-83 \%$ for six or more months; $7.1 \%$ for one to six months; and $9.8 \%$ for less than a month.

The participants had the following ethnic mix: $81.4 \%$ White, $10.6 \%$ Black, $1.6 \%$ Latino, 4.4\% Bi/Multiracial, and .9\% Asian. They ranged in age from 20 to 76 years, with a mean age of 47.4 years $(\mathrm{SD}=12.5)$. A majority of the participants had a posthigh-school education (84\%) - 15.9\% Associate's, 31.9\% Bachelor's, 27.4\% Master's, and $4.4 \%$ Doctorate degrees, while $4.4 \%$ had some college education. High school graduates made up $15.9 \%$ of the sample.

The respondents' yearly incomes were distributed as follows, $12.4 \%$ at least $\$ 150,000 ; 28.3 \%$ between $\$ 75,000$ and $\$ 149,000 ; 29.2 \%$ between $\$ 35,000$ and $\$ 74,999$; $13.3 \%$ between $\$ 20,000$ and $\$ 34,999$; and $15.9 \%$ less than $\$ 20,000$. A total of 63 participants (55.8\%) were married, 31 were single (27.4\%), nine divorced $(8 \%)$, five widowed (4.4\%), three separated (2.7\%), and one was in a domestic partnership (.9\%).

Respondent indicated the following religious affiliations, 23 (20.4\%) nondenominational/Evangelical, 23 (20.4\%) Seventh-day Adventists, 14 Baptist (12.4\%), 10 (8.8\%) Pentecostal/Charismatic, five (4.4\%) Catholic, four (3.5\%) as Methodist, and two $(1.8 \%)$ identified as Church of Christ. Eleven participants $(9.7 \%)$ indicated that they no 
longer identified with any organized religion, have left their church, or no longer claim to have faith. Four participants (3.5\%) claimed that their faith practices had changed since their experience of abuse (e.g., practicing "mystical Christianity" and "My spirituality has grown to include several different paths"). Overall, 10 participants (9\%) identified themselves as being minimally or not at all spiritual or religious, while six $(5.4 \%)$ considered themselves to be moderately spiritual or religious, and $96(84.9 \%)$ identified themselves as more or very spiritual or religious.

\section{Table 1}

\section{Participants Demographic Statistics}

\begin{tabular}{|c|c|c|}
\hline Demographic & $N$ & $\%$ \\
\hline \multicolumn{3}{|l|}{ Age } \\
\hline $20-34$ & 16 & 14.4 \\
\hline $35-49$ & 49 & 44.1 \\
\hline $50-64$ & 34 & 30.6 \\
\hline $65-76$ & 11 & 9.9 \\
\hline $\mathrm{M}=47.4 \quad \mathrm{SD}=12.5$ Range $20-76$ & & \\
\hline
\end{tabular}

Ethnicity

White 92

81.4

Black

12

10.6

Bi/Multiracial

$5 \quad 4.4$

Latino/a

2

Asian

1

.9

Caribbean

1

.9

Education

High School

Some College

Associate Degree

Bachelor's Degree

Master's Degree

Doctorate Degree 
Table 1-Continued

Yearly Household Income 14

12.4

$\$ 150$ or above

21

18.6

$\$ 100,000$ to $\$ 149,999$

11

9.7

$\$ 75,000$ to $\$ 99,999$

20

17.7

$\$ 50,000$ to $\$ 74,999$

13

11.5

$\$ 35,000$ to $\$ 49,999$

15

13.3

$\$ 20,000$ to $\$ 34,999$

18

15.9

Less than $\$ 20,000$

\begin{tabular}{lrr} 
Marital Status & & \\
Married & 63 & 55.8 \\
Single & 31 & 27.4 \\
Divorced & 9 & 8.0 \\
Widowed & 5 & 4.4 \\
Separated & 3 & 2.7 \\
Domestic Partnership & 1 & .9 \\
\hline & & \\
Religious Spiritual Affiliation & & 20.4 \\
Non-Denominational Evangelical & 23 & 20.4 \\
Seventh-day Adventist & 23 & 12.4 \\
Baptist & 14 & 8.8 \\
Pentecostal/Charismatic & 10 & 4.4 \\
Catholic & 5 & 3.5 \\
Methodist & 4 & 1.8 \\
Church of Christ & 2 & 1.8 \\
Presbyterian Church of America & 2 & 10.6 \\
Other Christian Denominations & 2 & .9 \\
Jewish & 12 & .9 \\
Hebrew Roots & 1 & .9 \\
Agnostic & 1 & .9 \\
Post-Christian & 1 & 9.7 \\
No Organized Religion & 1 & \\
\hline
\end{tabular}




\section{Reliability Estimates}

\section{Posttraumatic Growth Inventory}

The PTGI was used to assess PTG. The global scale consisted of 21 items that describe areas of potential growth following a traumatic event. The PTGI is divided into five subscales measuring specific areas of growth: (1) Relating to Others, (2) New Possibilities, (3) Personal Strength, (4) Spiritual Change, and (5) Appreciation of Life.

The Relating to Others subscale (seven items), which measures the degree to which respondents indicated having deeper interpersonal relationships, appeared highly reliable (Cronbach $\alpha .87$ ). This indicates that the Relating to Others subscale has few measurement errors and can consistently measure participants' sense of having deeper interpersonal relationships.

The New Possibilities scale (five items), which evaluates the degree to which respondents indicated having a readjustment of priorities, also appeared to have good reliability (Cronbach $\alpha .83$ ). This suggests that the New Possibilities subscale has few measurement errors and can consistently measure participants' sense of priority readjustments.

The Personal Strength subscale (four items), which measures respondents' sense of having gained an increased inner strength, appeared to have fairly good reliability (Cronbach $\alpha .80$ ). This suggests that the Personal Strength subscale has few measurement

errors and can measure, fairly consistently, the degree to which respondents felt they had greater inner strength.

The Spiritual Change subscale (two items), which measures the degree to which respondents acknowledged having a fuller spiritual or existential life, appeared 
moderately reliable (Cronbach $\alpha .74)$. This suggests that the Spiritual Change subscale has some measurement errors and can measure the degree to which respondents' sense of spirituality has increased with moderate consistency.

The Appreciation of Life subscale (three items), which measures participants' sense of an increased appreciation for life, appeared moderately reliable (Cronbach $\alpha$.78). This suggests that the Appreciation of Life subscale has some measurement errors and can measure the degree to which respondents' appreciation for life has increased with moderate consistency. See Table 2.

\section{Brief RCOPE}

Religious and spiritual coping was assessed using the Brief RCOPE. The global scales consisted of 14 items and contained two subscales: Negative Religious Coping (NRC) and Positive Religious Coping (PRC). The seven-item NRC subscale, which identifies tension, conflict, discontent, and/or a struggle with God, demonstrated good reliability with a Cronbach $\alpha$ of .84. This suggests that the NRC has few measurement errors and can measure negative religious coping with consistency. The PRC subscale (seven items), which identifies a secure relationship with God/Higher Power and a sense that life is meaningful, demonstrated high reliability with a Cronbach $\alpha$ of .87. This suggests that the PRC also has few measurement errors and can consistently measure positive religious coping. See Table 2. 
Table 2

Reliability Coefficients for Scales

\begin{tabular}{llcc}
\hline & Subscale & No. of Items & Cronbach's $\alpha$ \\
\hline \multirow{2}{*}{ PTGI } & Relating to Others & 7 & .87 \\
& New Possibilities & 5 & .83 \\
& Personal Strength & 4 & .80 \\
& Spiritual Change & 2 & .74 \\
& Appreciation of Life & 3 & .78 \\
Brief RCOPE & Negative Religious Coping & 7 & .84 \\
& Positive Religious Coping & 7 & .87 \\
\hline
\end{tabular}

* Cronbach's $\alpha$ coefficient is internally reliable at $\geq .7$.

\section{Levels of Distress, Spiritual Coping, and Posttraumatic Growth}

\section{Distress}

Distress shortly after the abuse, as remembered by participants, was measured using four one-item subscales of the ET: Distress, Anxiety, Depression, and Anger. The Distress subscale of the ET had a mean statistic of 9.20 out of 10 , with a standard deviation of 1.78 . The distribution for this subscale was highly negatively skewed $(-2.79)$ and had a median of 10, indicating that participants experienced very high levels of distress shortly after the abuse. The mean statistic for the Anxiety subscale was 9.16 out of $10(\mathrm{SD}=1.63)$. This distribution was also highly negatively skewed $(-2.41)$ with most of the values on the high end of the scale (median $=10)$. Thus, survivors experienced very high levels of anxiety shortly after the abuse. The Depression subscale had a mean of 8.71 out of $10(\mathrm{SD}=2.21)$. This distribution had a high negative skew $(-1.78)$ with a median of 10 , suggesting a very high level of depression in survivors shortly after the abuse. Finally, the mean for the Anger subscale was 7.80 out of $10(\mathrm{SD}=2.86)$, with a high negative skew (-1.05) and a median of 10 . This indicates that most of the values 
were on the high end of the scale, and a high level of anger was experienced by survivors shortly after the abuse. See Table 3 .

Table 3

Subscale Means, Medians, Standard Deviations, and Skewness

\begin{tabular}{|c|c|c|c|c|c|c|}
\hline & Min & Max & $\mathrm{M}$ & SD & Med & $\begin{array}{r}\text { Skew- } \\
\text { ness }\end{array}$ \\
\hline Q22 How emotionally upset - Distress & 1.0 & 10.0 & 9.20 & 1.78 & 10.00 & -2.79 \\
\hline Q22 How emotionally upset - Anxiety & 1.0 & 10.0 & 9.16 & 1.63 & 10.00 & -2.41 \\
\hline Q22 How emotionally upset -Depression & 1.0 & 10.0 & 8.71 & 2.21 & 10.00 & -1.78 \\
\hline Q22 How emotionally upset - Anger & 1.0 & 10.0 & 7.80 & 2.86 & 10.00 & -1.05 \\
\hline Positive coping (PRC) & 1.00 & 4.00 & 2.71 & 0.83 & 2.86 & -0.51 \\
\hline Negative coping (NRC) & 1.00 & 4.00 & 2.47 & 0.84 & 2.29 & 0.18 \\
\hline Relating to others & 1.00 & 6.00 & 3.39 & 1.31 & 3.43 & 0.01 \\
\hline New possibilities & 1.00 & 6.00 & 4.06 & 1.32 & 4.20 & -0.55 \\
\hline Personal strengths & 1.00 & 6.00 & 3.93 & 1.29 & 4.00 & -0.27 \\
\hline Spiritual change & 1.00 & 6.00 & 3.87 & 1.67 & 4.00 & -0.34 \\
\hline Appreciation of life & 1.00 & 6.00 & 4.23 & 1.40 & 4.33 & -0.83 \\
\hline
\end{tabular}

\section{Spiritual Coping}

Positive and negative styles of spiritual coping were measured using the two subscales of the Brief RCOPE: Positive Religious Coping (PRC) and Negative Religious Coping (NRC). The PRC subscale of the Brief RCOPE had a mean statistic of 2.71 out of 4 , with a standard deviation of .83 . This suggests that participants were more likely to use positive spiritual coping between "somewhat" and "quite a bit," with a greater tendency toward using positive coping "quite a bit." The NRC had a mean of 2.47 out of four (SD $=0.84)$. This statistic suggests that participants were most likely to use negative spiritual coping some of the time (i.e., somewhat) or most of the time (i.e., quite a bit), with a tendency toward using negative coping some of the time. See Table 3.

On the PRC subscale, between $31.9 \%$ and $69.9 \%$ of participants responded: "quite a bit" or "a great deal" to the following items: Sought God's/Higher Power's love and 
care (69.9\%); Looked for a stronger connection with God/Higher Power (69\%); Asked forgiveness for my sins (64.6\%); Tried to put my plans into action together with God/Higher Power (58.4\%); Sought help from God/Higher Power in letting go of my anger (50.4\%); and Focused on religion to stop worrying about my problems $(31.9 \%)$. See Table 4.

\section{Table 4}

\section{RCOPE PRC Subscale Mean, Standard, Deviation, and Percent by Item}

\begin{tabular}{lllll}
\hline Item & $\mathrm{N}$ & $\mathrm{M}$ & $\mathrm{SD}$ & $\%^{\mathrm{a}}$ \\
\hline 02-Sought God's/Higher Power's love and care. & 113 & 3.03 & 1.08 & 69.91 \\
01-Looked for a stronger connection with God/Higher Power. & 113 & 2.97 & 1.08 & 69.03 \\
06-Asked forgiveness for my sins. & 113 & 2.90 & 1.14 & 64.60 \\
04-Tried to put my plans into action together with God/Higher & 113 & 2.76 & 1.08 & 58.43 \\
Power. & & & & \\
05-Tried to see how God/Higher Power might be trying to & 113 & 2.73 & 1.19 & 58.41 \\
strengthen me in this situation. & & & & \\
$\begin{array}{l}\text { 03-Sought help from God/Higher Power in letting go of my } \\
\text { anger. }\end{array}$ & 113 & 2.58 & 1.13 & 50.44 \\
07-Focused on religion to stop worrying about my problems. & 113 & 1.97 & 1.06 & 31.86 \\
\hline ao “quite a bit” and “a great deal.” & & & &
\end{tabular}

Participants who responded "quite a bit" or "a great deal" on the items of the NRC subscale ranged from $28.3 \%$ to $77.9 \%$. These items included: Wondered why my church had abandoned me (77.9\%); Wondered whether God/Higher Power had abandoned me (56.6\%); Questioned God's (Higher Power's) love for me (49.6\%); Felt punished by God/Higher Power (41.6\%); Questioned the power of God/Higher Power (37.2\%); Wondered what I did for God/Higher Power to punish me (37.2\%); Decided the Devil made this happen (28.3\%). See Table 5. 
Table 5

RCOPE NRC Subscale Mean, Standard Deviation, and Percent by Item

\begin{tabular}{llllc}
\hline Item & $\mathrm{N}$ & $\mathrm{M}$ & $\mathrm{SD}$ & $\%^{\mathrm{a}}$ \\
\hline 12-Wondered whether my church had abandoned me. & 113 & 3.39 & 1.00 & 77.88 \\
08-Wondered whether God/Higher Power had abandoned me. & 113 & 2.70 & 1.22 & 56.64 \\
11-Questioned God's (Higher Power's) love for me. & 113 & 2.53 & 1.21 & 49.56 \\
09-Felt punished by God/Higher Power. & 113 & 2.24 & 1.27 & 41.59 \\
14-Questioned the power of God (Higher Power). & 113 & 2.21 & 1.27 & 37.19 \\
10-Wondered what I did for God/Higher Power to punish me. & 113 & 2.12 & 1.24 & 37.17 \\
13-Decided the Devil made this happen. & 113 & 2.08 & 1.03 & 28.34 \\
\hline
\end{tabular}

a \% "quite a bit" and "a great deal."

\section{Posttraumatic Growth}

PTG was measured using the PTGI, which contained five subscales: Relating to Others subscale (7 items), New Possibilities (5 items), Personal Strength (4 items), Spiritual Change (2 items), and Appreciation of Life (3 items). Relating to Others had a mean statistic of 3.39 out of $6(\mathrm{SD}=1.31)$, indicating that participants experienced growth in this area on average from a small to moderate degree. New Possibilities had a mean of 4.06 out of $6(\mathrm{SD}=1.32)$, suggesting that survivors in our sample experienced growth in this area to a moderate degree, on average, after their abuse. Personal Strength had a mean of 3.93 out of $6(\mathrm{SD}=1.29)$. This indicates that survivors experienced growth in the area of personal strength to a moderate degree on average as a result of their crisis. Spiritual Change had a mean of 3.87 out of $6(\mathrm{SD}=1.67)$, suggesting that participants were likely to experience growth in the area of spiritual change from a small to a moderate degree (on average), with participants more likely to experience a moderate degree of growth. Finally, Appreciation of Life had a mean of 4.23 out of $6(\mathrm{SD}=1.40)$. This suggests that, on average, growth in this area was experienced by survivors from a 
moderate to a great degree, with survivors being more likely to experience this growth to a moderate degree. See Table 3.

Participants who claimed to have experienced the changes presented by the survey from a moderate to a very great degree ranged from $35.40 \%$ to $88.50 \%$. Of the seven items on the Relating to Others subscale, item 15 ("I have more compassion for others") had the greatest endorsement, receiving $81.42 \%$ of responses between the moderate to very great degree range. Item 20 ("I learned a great deal about how wonderful people are") was endorsed the least, with $35.40 \%$ of respondents claiming they experienced this change from a moderate to a very great degree. See Table 6.

\section{Table 6}

\section{PTGI Relating to Others Subscale Mean, Standard Deviation, and Percent by Item}

\begin{tabular}{lcccc}
\hline Item & $\mathrm{N}$ & $\mathrm{M}$ & $\mathrm{SD}$ & $\%^{\mathrm{a}}$ \\
\hline 15-I have more compassion for others. & 113 & 4.68 & 1.61 & 81.42 \\
16-I put more effort into my relationships. & 113 & 3.75 & 1.71 & 59.36 \\
09-I am more willing to express my emotions. & 113 & 3.51 & 1.78 & 53.16 \\
06-I more clearly see that I can count on people in times of trouble. & 113 & 2.99 & 1.81 & 43.36 \\
08-I have a greater sense of closeness with others. & 113 & 3.06 & 1.77 & 41.67 \\
21-I better accept needing others. & 113 & 2.98 & 1.65 & 40.71 \\
20-I learned a great deal about how wonderful people are. & 113 & 2.76 & 1.78 & 35.40
\end{tabular}

\% "quite a bit" and "a great deal."

Of the five items on the New Possibilities subscale of the PTGI, item 7 ("I established a new path for my life") received the greatest endorsement, with $77.88 \%$ of responses in the moderate to very great degree range. The least-endorsed item on this 
subscale was item 11 ("I am able to do better things with my life"), with $57.59 \%$ of responses in the moderate to very great degree range. See Table 7.

\section{Table 7}

\section{PTGI New Possibilities Subscale Mean, Standard Deviation, and Percent by Item}

\begin{tabular}{lcccc}
\hline Item & $\mathrm{N}$ & $\mathrm{M}$ & $\mathrm{SD}$ & $\%^{\mathrm{a}}$ \\
\hline 07-I established a new path for my life. & 113 & 4.59 & 1.54 & 77.88 \\
17-I am more likely to try to change things that need changing. & 113 & 4.14 & 1.63 & 73.45 \\
03-I developed new interests. & 113 & 3.99 & 1.63 & 66.44 \\
14-New opportunities are available which wouldn't have been & 113 & 3.76 & 1.91 & 60.21 \\
otherwise. & & & & \\
11-I am able to do better things with my life. & 113 & 3.82 & 1.79 & 57.59 \\
\hline
\end{tabular}

a\% "quite a bit" and "a great deal."

Of the four items on the Personal Strength subscale of the PTGI, item 19 ("I discovered that I'm stronger than I thought I was") and item 4 ("I have a greater feeling of self-reliance") received the greatest endorsement, with both items obtaining $76.99 \%$ of responses in the moderate, great, or very great degree range. Item 12 ("I am better able to accept the way things work out") was endorsed the least on this subscale, receiving $57.59 \%$ of responses in the moderate to very great degree range. See Table 8 .

\section{Table 8}

PTGI Personal Strength Subscale Mean, Standard Deviation, and Percent by Item

\begin{tabular}{lllll}
\hline Item & $\mathrm{N}$ & $\mathrm{M}$ & $\mathrm{SD}$ & $\%^{\mathrm{a}}$ \\
\hline 19-I discovered that I'm stronger than I thought I was. & 113 & 4.49 & 1.72 & 76.99 \\
04-I have a greater feeling of self-reliance. & 113 & 4.36 & 1.50 & 76.99 \\
10-I know better that I can handle difficulties. & 113 & 4.12 & 1.68 & 69.03 \\
12-I am better able to accept the way things work out. & 113 & 3.71 & 1.70 & 57.59 \\
\hline
\end{tabular}

a\% "quite a bit" and "a great deal."

There were two items in the Spiritual Change subscale of the PTGI. Of these, item 5 ("I have a better understanding of spiritual matters") was endorsed $72.57 \%$ of the time 
in the moderate, great, or very great degree range. Item 18 ("I have a stronger religious faith") received $58.50 \%$ of responses in the moderate to very great degree range. See

Table 9.

Table 9

PTGI Spiritual Change Subscale Mean, Standard Deviation, and Percent by Item

\begin{tabular}{llllc}
\hline Item & $\mathrm{N}$ & $\mathrm{M}$ & $\mathrm{SD}$ & $\%^{\mathrm{a}}$ \\
\hline 05-I have a better understanding of spiritual matters. & 113 & 4.16 & 1.27 & 72.57 \\
18-I have a stronger religious faith. & 113 & 3.59 & 2.02 & 58.50 \\
\hline
\end{tabular}

a\% "quite a bit" and "a great deal."

Of the three items in the Appreciation of Life subscale, item 1 ("I changed my priorities about what is important in life") received the greatest endorsement, with $88.50 \%$ of respondents claiming that they experienced this change to a moderate, great, or very great degree. Item 13 ("I can better appreciate each day") was endorsed the least, with $66.44 \%$ of respondents claiming they had experienced this change from a moderate to a very great degree. See Table 10.

\section{Table 10}

PTGI Appreciation of Life Subscale Mean, Standard Deviation, and Percent by Item

\begin{tabular}{lcccc}
\hline Item & $\mathrm{N}$ & $\mathrm{M}$ & $\mathrm{SD}$ & $\%^{\mathrm{a}}$ \\
\hline 01-I changed my priorities about what is important in life. & 113 & 4.71 & 1.40 & 88.50 \\
02-I have a greater appreciation for the value of my own life. & 113 & 4.25 & 1.79 & 71.68 \\
13-I can better appreciate each day. & 113 & 3.74 & 1.81 & 66.44 \\
\hline
\end{tabular}

a\% "quite a bit" and "a great deal." 


\section{Results by Research Question}

\section{Question 1: Context of Abuse}

Table 11 summarizes the findings for the Context of Abuse survey in our sample. Questions regarding the age at which the abuse occurred revealed that most survivors experienced the abuse in adulthood (61.1\%), between the ages of 27 and 59; $31 \%$ during young adulthood (between 19 and 26 years old); 24.8\% during adolescence (between 11 and 18 years old); and $6.2 \%$ during childhood (between four and 10 years of age).

Regarding the frequency of the abuse, $48.7 \%$ of respondents reported that it occurred too many times for them to remember, $23.9 \%$ reported six or more times, $18.6 \%$ reported two to five times, and $7.1 \%$ reported one time. Ninety-two percent of the participants stated that the abuse continued over a period of time. The time over which the abuse occurred was identified as under six months (11.5\%), six months to a year $(19.5 \%)$, one to two years $(20.4 \%)$, three to five years $(16.8 \%)$, or more than five years $(23.9 \%)$.

Participants identified the abuse as fondling or any touching used to invoke sexual feelings $(N=93,82.3 \%)$; sexually explicit, suggestive, or humiliating comments or language $(N=81,71.7 \%)$; indecent exposure $(N=45,39.8 \%)$; exposure to masturbation or other sexual acts $(N=42,37.2 \%)$; coercion or encouragement to masturbate $(N=33$, $29.2 \%)$; penetrative sexual acts $(N=64,56.6 \%)$; pornography viewing $(N=14,12.4 \%)$; creating pornographic images or video $(N=21,18.6 \%)$; and/or forced prostitution $(N=2$, $1.8 \%)$. Approximately $19.4 \%$ of participants $(N=22)$ identified other abusive acts, such as threatened rape and coercive group sex. Most respondents indicated being sexually abused by one religious leader $(N=89 ; 79.4 \%)$, while others $(N=23 ; 20.5 \%)$ reported being abused by more than one religious leader. 
Respondents identified their abuser as a Pastor, Priest, Bishop, or Rabbi $(N=102$, 90.2\%); an Elder or Deacon $(N=22,19.4 \%)$; or other religious leader (e.g., Bible College or Seminary Professors, Navy or other Chaplain, and Teacher/Pathfinder leader) $(N=9,7.9 \%)$

In the two years following the abuse, many respondents reported seeking and receiving counseling $(62.8 \%)$. The duration of counseling was under six months $(17.7 \%)$, six months to a year $(12.4 \%)$, one to two years $(8.8 \%)$, or more than two years $(25.7 \%)$. See Table 11.

\section{Table 11}

\section{Context of Abuse Statistics}

\begin{tabular}{lrr}
\hline Context & $N$ & $\%$ \\
\hline At what age did the abuse occur? & 7 & 6.2 \\
Childhood (4-10) & 28 & 24.8 \\
Adolescence (11-18) & 35 & 31.0 \\
Young Adulthood (19-26) & 69 & 61.1 \\
Adulthood (27-59) & 8 & \\
\hline How many times did the abuse occur? & 21 & 18.6 \\
1 time & 27 & 23.9 \\
2-5 times & 55 & 49.5 \\
6 or more times & & \\
Too many times to remember/can't remember & 104 & 92.0 \\
Did the abuse occur over a period of time? & 8 & 7.1 \\
Yes & & \\
No & 13 & 11.5 \\
Under 6 months & 22 & 19.5 \\
6 months to 1 year & 23 & 20.4 \\
1 to 2 years & 19 & 16.8 \\
3 to 5 years & 27 & 23.9 \\
More than 5 years & & \\
\hline
\end{tabular}


Table 11-Continued

Which of the following describes the nature of the abuse?

$\begin{array}{lll}\text { Fondling or any touching used to evoke sexual feelings } & 93 & 82.3\end{array}$

Sexually explicit, suggestive, or humiliating comments or $\quad 81 \quad 71.7$

language

$\begin{array}{lll}\text { Indecent exposure } & 45 & 39.8\end{array}$

$\begin{array}{lll}\text { Exposure to masturbation or other sexual acts } & 42 & 37.2\end{array}$

$\begin{array}{lll}\text { Coercion or encouragement to masturbate } & 33 & 29.2\end{array}$

$\begin{array}{lll}\text { Penetrative sexual acts } & 64 & 56.6\end{array}$

$\begin{array}{lll}\text { Pornography viewing } & 14 & 12.4\end{array}$

$\begin{array}{lll}\text { Creating pornographic images or video } & 21 & 18.6\end{array}$

$\begin{array}{lrr}\text { Forced prostitution } & 2 & 1.8\end{array}$

Other acts (e.g., threatened rape, coercive group sex, etc) $\quad 91 \quad 80.5$

How many religious leaders abused you?

$\begin{array}{lll}\text { One } & 89 & 78.8\end{array}$

$\begin{array}{lll}\text { Two } & 10 & 8.8\end{array}$

$\begin{array}{lll}\text { Three } & 7 & 6.2\end{array}$

Four $\quad 1 \quad r$

Five $\quad 0 \quad 0$

$\begin{array}{lll}\text { Six } & 2 & 1.8\end{array}$

$\begin{array}{lll}\text { More than six } & 3 & 2.7\end{array}$

Who abused you?

Pastor, Priest, Bishop, or Rabbi $\quad 102 \quad 90.2$

$\begin{array}{lll}\text { Elder or Deacon } & 22 & 19.4\end{array}$

$\begin{array}{lll}\text { Other religious leader (e.g., Bible College/Seminary } & 9 & 7.9\end{array}$

Professor, Navy Chaplain, Teacher/Pathfinder leader, etc.)

In the 2 years following the abuse, did you seek or receive counseling?

$\begin{array}{lll}\text { Yes } & 71 & 62.8\end{array}$

$\begin{array}{lll}\text { No } & 40 & 35.4\end{array}$

If yes, how long did the counseling last?

$\begin{array}{lll}\text { Under } 6 \text { months } & 20 & 17.7\end{array}$

6 months to 1 year $\quad 14 \quad 12.4$

1 to 2 years $\quad 10 \quad 8.8$

\begin{tabular}{lll} 
More than 2 years & 29 & 25.7 \\
\hline
\end{tabular}

The results for Questions 2-4 were examined using Path Analysis and are presented in the following segment. Path Analysis is a multiple regression analysis that 
allows researchers to evaluate hypothesized predictive models by examining the relationships between the variables depicted in the data (Meyers et al., 2013). The fundamental objective in this process is to determine whether a hypothesized model is an adequate fit to the given data. Numerous fit indices have been used in the literature to determine the nature of the fit between the hypothesized model and the data. In this study, the following fit indices are used: Chi-square, Goodness of Fit Index, Comparative Fit Index, Normed Fit Index, Root Mean Square Error of Approximation, and Standard Root Mean Square Residual.

A Chi-square test examines the extent to which expectations agree with the observed data (Meyers, et al., 2013). A non-significant Chi-square value suggests that the model is a good fit to the data. The Goodness of Fit Index evaluates the discrepancy explained by the estimated covariance of the population (Tabachnick \& Fidell, 2007). A target value that is equal to or greater than .90 indicates a good fit. The Comparative Fit Index analyzes discrepancies between the hypothesized model and the data to determine goodness of fit. The Normed Fit Index measures goodness of fit by comparing the hypothesized model to a model with uncorrelated variables. Target values for the Comparative Fit Index and the Normed Fit Index are .95 for a good fit and .90 for an acceptable fit (Meyers et al., 2013). The Root Mean Square Error of Approximation is a goodness of fit measure that seeks to determine whether the model is a close or approximate fit, if not an exact fit. Target values are .08 and are reported with confidence intervals. The Standard Root Mean Square Residual calculates the difference between the predicted and observed correlation in order to determine goodness of fit. Values that range between 0 and .08 indicate an acceptable fit (Hu \& Bentller, 1999). 


\section{Question 2: Effects of Spiritual Coping on Posttraumatic Growth}

This study predicted that spiritual/religious coping styles would directly affect the level of PTG (PTG) in female survivors of SARL. The present path analysis focused on positive and negative coping styles as predictors of PTG in five domain areas: relating to others, new possibilities, personal strength, spiritual change/growth, and appreciation of life. These are configured into the hypothesized model shown in Figure 3.

\section{Figure 3}

Path Diagram of the Hypothesized Model Between Spiritual Coping and Growth

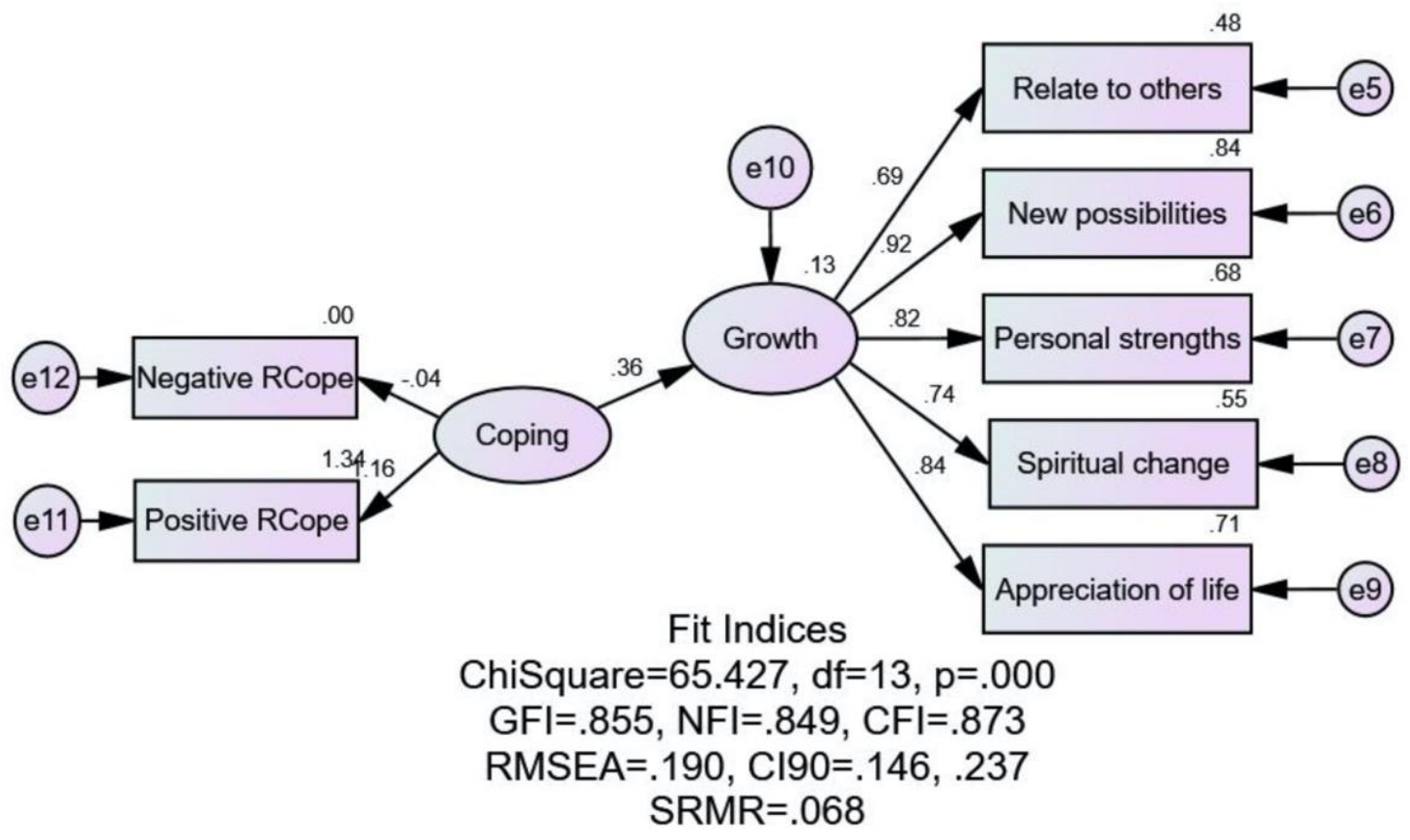

The model was evaluated using International Business Machines Statistical Package for Social Sciences Analysis of Moment Structures (IBM SPSS AMOS) version 23. The Chi-square assessing the model fit was found to be statistically significant at a value of $65.427(13, N=113), p=.000$. Thus, the model did not appear to be a good fit to the data. This was supported by the Goodness of Fit Index (.855), Normed Fit Index (.849), and Comparative Fit Index (.873) that use a target value of $>0.95$ to indicate a 
good fit and a minimum of $>.90$ for an acceptable fit. These fit indices, including the Root Mean Square Error of Approximation (target value of $<0.08$ for a good fit) of .190 with a $90 \%$ confidence interval of .146 to .237 suggested that the model was not a good fit. However, the Standard Root Mean Square Residual of .068 fell in the target window (between 0 and .08 target window) and was the only index suggesting the model was an acceptable fit.

Given these findings, modifications to improve model fit were flagged by examining the modification indices. These indices suggested that the errors associated with personal strength and relating to others covary $(\mathrm{MI}=36.65)$ and that coping correlates with spiritual change $(\mathrm{MI}=8.25)$. In addition, the model showed that in our sample, Negative RCOPE (NRC) did not contribute to coping and that coping was primarily made up of Positive RCOPE (PRC). Based on this information, three changes were made to the model that make theoretical sense. First, NRC was removed from the model, and PRC, as the main contributor to coping in our sample, was retained. Second, a correlation was made between the error associated with Relating to Others and the error associated with Personal Strength. It makes sense that an increase in the quality of interpersonal relationships provides the support individuals need to feel increased personal strength. Third, a path from the error associated with Coping to the error associated with Spiritual Change was added. It makes sense that a measure of spiritual coping such as the RCOPE would be closely correlated with the subscale of the PTGI that measures spiritual change.

The modified model was then evaluated using IBM SPSS Amos. The results of the re-specified model found a significant improvement over the initial model. The Chi- 
square in this analysis was not statistically significant with a value of $10.147(7, N=$ $113), p=.180$, suggesting that the model was a good fit to the data. The Goodness of Fit Index (.970), NFI (.976), and Comparative Fit Index (.992) all yielded values above .90, while the Root Mean Square Error Approximation obtained a value of .063 with a 90\% confidence interval of .000 to .142 . The target value for this index was $<.08$ for goodness of fit. All of these fit indices provided additional evidence of the model's goodness of fit to the data. Regression coefficients for the paths in this re-specified model are reported in Table 12. The analysis found that RCOPE was a significant predictor of PTG, with a standardized regression weight of .403 . The model was able to account for $16 \%$ of the variance in PTG reported by survivors. This variance was due to the direct effects of PRC. This outcome supports the first hypothesis of this study which states that survivors with positive spiritual coping styles will experience higher levels of PTG than those who use negative spiritual coping styles. The standardized path coefficients for the respecified model are presented in Figure 4. Model summaries for the initial and respecified models are presented in Table 13.

Table 12

Path Coefficients Between RCOPE and PTG (Re-Specified Model)

\begin{tabular}{|c|c|c|c|c|c|c|}
\hline Path & & $\mathrm{b}$ & $\mathrm{SE}$ & $\beta$ & $\mathrm{CR}$ & $\mathrm{p}$ \\
\hline Growth & $<---$ PosRCope & 0.403 & 0.103 & 0.403 & 3.925 & $<.001$ \\
\hline Relate & Growth & 1.000 & & 0.631 & & \\
\hline NewPossib & Growth & 1.500 & 0.195 & 0.941 & 7.709 & $<.001$ \\
\hline PerStrength & Growth & 1.221 & 0.119 & 0.785 & 10.222 & $<.001$ \\
\hline SpiritChange & Growth & 1.303 & 0.215 & 0.644 & 6.071 & $<.001$ \\
\hline AppreciateLife & Growth & 1.446 & 0.197 & 0.854 & 7.357 & $<.001$ \\
\hline
\end{tabular}




\section{Figure 4}

Re-Specified Path Model Relating Positive Spiritual Coping and Posttraumatic Growth

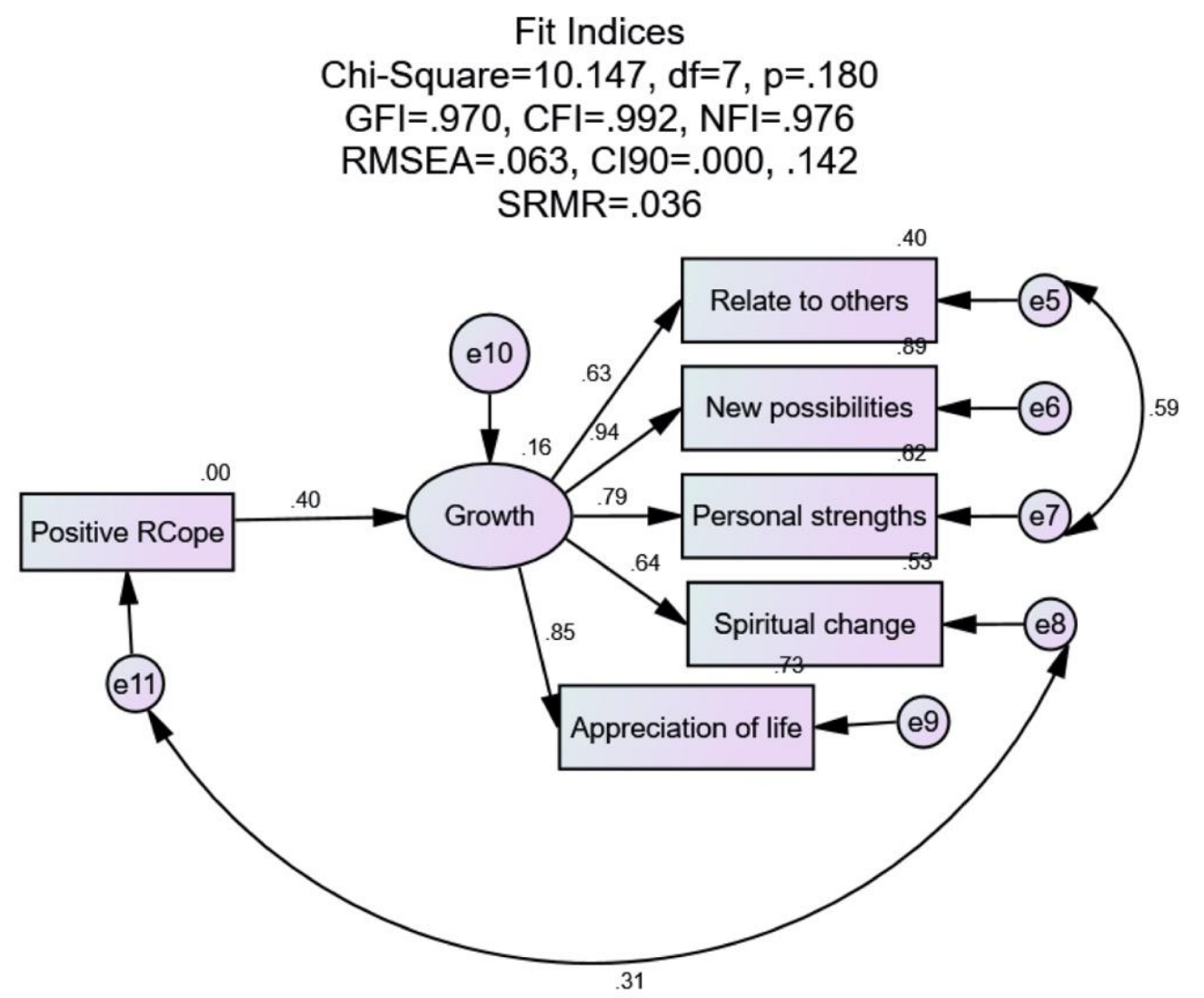

Table 13

Model Summaries for RCOPE and PTG

\begin{tabular}{lrrrrrrrl}
\hline & CMIN & GFI & NFI & CFI & RMSEA & SRMR & N & df \\
\hline Model 1 Initial Model & 65.43 & .855 & .849 & .873 & .190 & .068 & 113 & 13 \\
Model 2 Re-Specified Model & 10.15 & .970 & .976 & .992 & .063 & .036 & 113 & 7 \\
\hline Target Values & & $>.95$ & $>.95$ & $>.95$ & $<.08$ & $0-.08$ & &
\end{tabular}

\section{Question 3: Effects of Distress on Posttraumatic Growth}

This study predicted that PTG would be directly affected by the distress levels of female survivors of SARL. Thus, a path analysis was conducted which focused on selfidentified distress, anxiety, depression, and anger as predictors of PTG in the five domain 
areas: relating to others, new possibilities, personal strength, spiritual change/growth, and appreciation of life. An asymptotically distribution-free analysis was conducted due to the severe skewness of some of the distress indicators. These are configured into the hypothesized model shown in Figure 5.

\section{Figure 5}

Hypothesized Path Model Relating Distress and Posttraumatic Growth

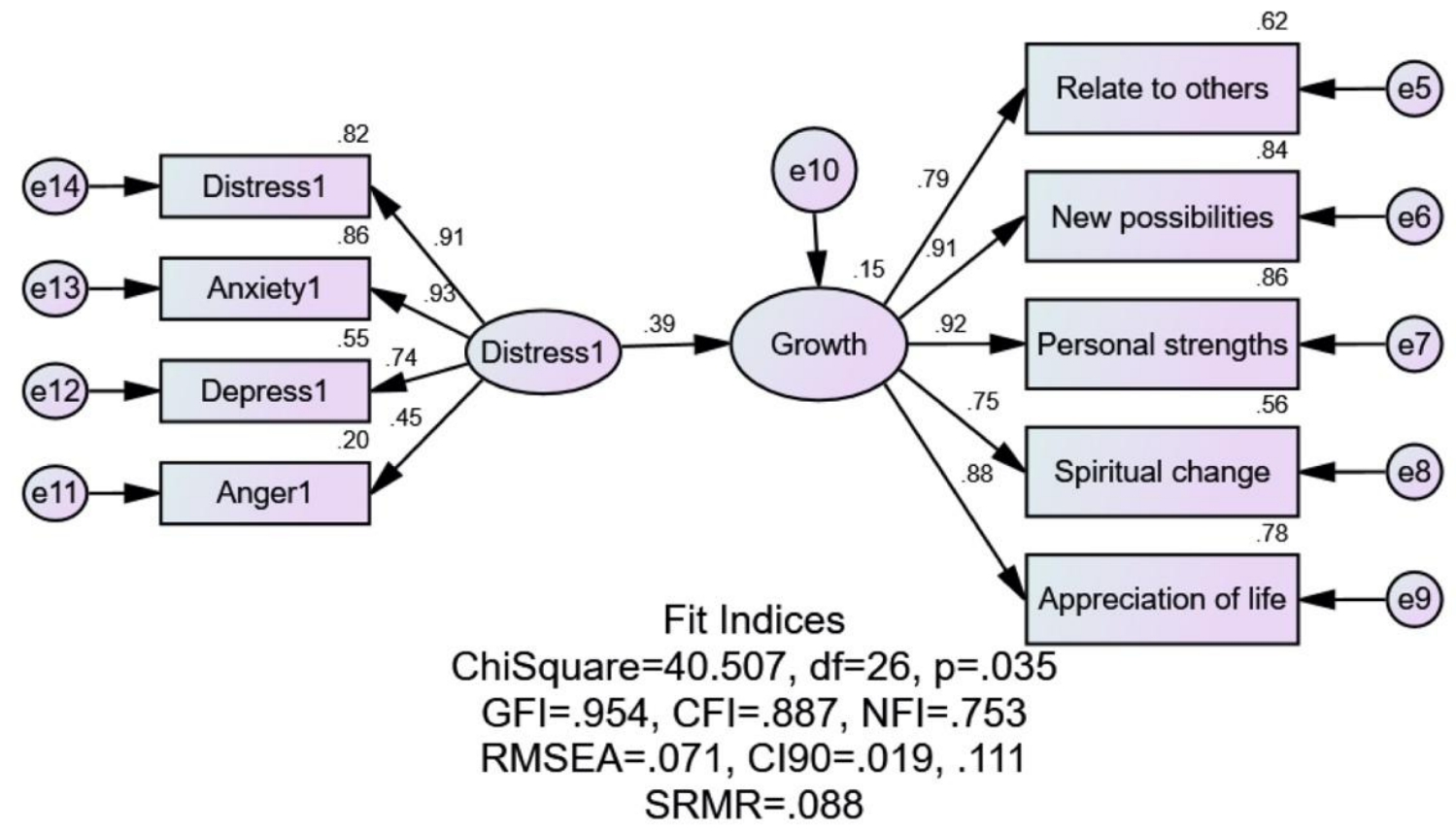

The model was evaluated using IBM SPSS Amos. The Chi-square assessing model fit was statistically significant at a value of $40.507(26, N=113), p=.035$, suggesting that the model was not a good fit to the data. While the Goodness of Fit Index (.95) and the Root Mean Square Error Approximation (.071; 90\% confidence interval of .019 to .111) suggested a good fit, other fit indices suggested that the model was not quite an acceptable fit (Comparative Fit Index $=.89$; Normed Fit Index $=.75$; Standard Root Mean Square Residual $=.088)$. 
Modifications to improve the model were flagged by examining the modification indices. These indices suggested that the error associated with distress and the error associated with personal strength covary $(\mathrm{MI}=4.48)$. Possibly that the presence of distress and the human inclination to find relief, compels survivors to draw upon inner resources they did not know they had or develop new, thus increasing their perceived personal strength. Thus, a correlation was made between the error associated with distress and the error associated with personal strength.

The results of the re-specified model found a slight improvement over the initial model. In this analysis, the Chi-square value of $34.248(25, N=113)$ was not statistically significant $(p=.103)$ and the Root Mean Square Error Approximation obtained a value of .057 with a $90 \%$ confidence interval of .000 to .101 , suggesting that the model was a good fit to the data. Additional fit indices (Goodness of Fit Index =.96; Comparative Fit Index $=.93$ ) provided evidence of an acceptable fit, with values at .90 or above. We used target values of .95 or above for these indices. However, .90 is considered acceptable by some researchers (Meyers et al., 2013). Notwithstanding, the Normed Fit Index (.079, with a target value of $>.95)$ and Standard Root Mean Square Residual (.088, with a target window between 0 and .08) suggest the model was not quite an acceptable fit. The path analysis found that the perceived distress of survivors soon after the abuse took place was a significant predictor of PTG with a standardized regression weight of .416. See Table 14 for standardized regression weights in the re-specified model. The model was able to account for $17 \%$ of the variance in PTG reported by survivors. The Path coefficients for the re-specified model are presented in Figure 6. Model summaries for the initial and respecified models are presented in Table 15. 
Table 14

Path Coefficients Between Distress and PTG (Re-Specified Model)

\begin{tabular}{|c|c|c|c|c|c|c|}
\hline Path & & $\mathrm{b}$ & $\mathrm{SE}$ & $\beta$ & $\mathrm{CR}$ & $\mathrm{p}$ \\
\hline Growth & $<---$ Distress 1 & 0.320 & 0.059 & 0.416 & 5.385 & $<.001$ \\
\hline Relate & $<---\quad$ Growth & 1.000 & & 0.788 & & $<.001$ \\
\hline NewPossib & $<---\quad$ Growth & 1.205 & 0.074 & 0.921 & 16.343 & $<.001$ \\
\hline PerStrength & $<---\quad$ Growth & 1.207 & 0.075 & 0.930 & 16.130 & $<.001$ \\
\hline SpiritChange & $<---\quad$ Growth & 1.204 & 0.104 & 0.737 & 11.580 & $<.001$ \\
\hline AppreciateLife & $<---\quad$ Growth & 1.264 & 0.102 & 0.898 & 12.373 & $<.001$ \\
\hline Q22Anger_1 & <--- Distress1 & 1.000 & & 0.479 & & $<.001$ \\
\hline Q22Depress_1 & $<---$ Distress1 & 1.206 & 0.221 & 0.701 & 5.447 & $<.001$ \\
\hline Q22Anxiety_1 & $<---$ Distress 1 & 1.104 & 0.138 & 0.942 & 8.004 & $<.001$ \\
\hline Q22Distress_1 & $<---$ Distress 1 & 1.121 & 0.155 & 0.902 & 7.235 & $<.001$ \\
\hline
\end{tabular}

Figure 6

Re-Specified Path Model Relating Distress and Posttraumatic Growth

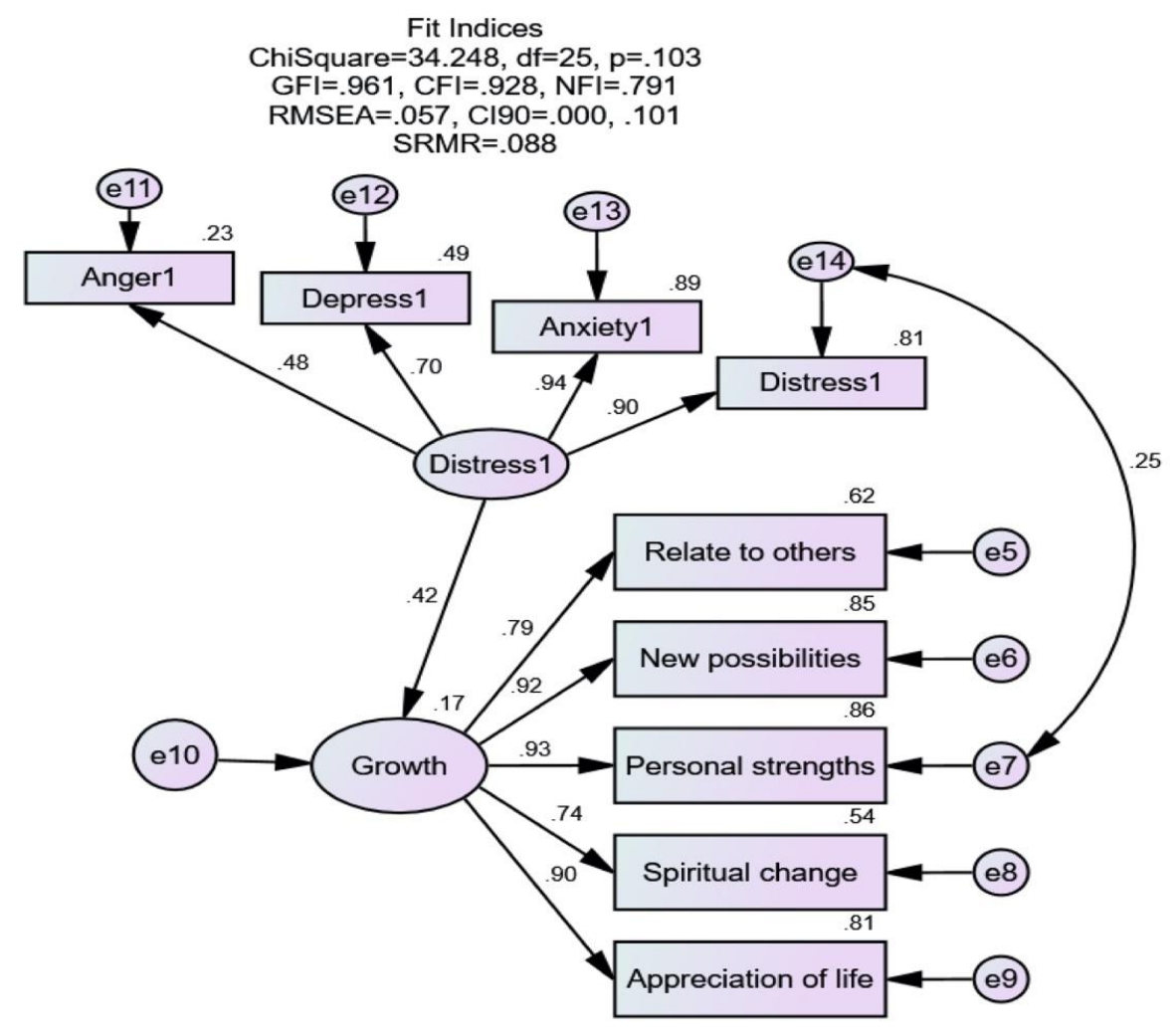




\section{Table 15}

Model Summaries for Distress and PTG

\begin{tabular}{lrrrrrrrr}
\hline & CMIN & GFI & NFI & CFI & RMSEA & SRMR & N & df \\
\hline Model 1 Initial Model & 40.51 & .954 & .753 & .887 & .071 & .088 & 113 & 26 \\
Model 2 Re-Specified Model & 34.25 & .961 & .791 & .928 & .052 & .088 & 113 & 25 \\
\hline Target Values & & $>.95$ & $>.95$ & $>.95$ & $<.08$ & $0-.08$ & &
\end{tabular}

\section{Question 4: Spiritual Coping as a Mediator Between Distress}

\section{and Posttraumatic Growth}

The present study examined PTG as the latent outcome variable in 113 female survivors of SARL using the five dimensions (subscales) identified by Tedeschi and Calhoun (1996) in the PTGI: relating to others, new possibilities, personal strength, spiritual change/growth, and appreciation of life. Distress, indicated by four subscales (i.e., distress, anxiety, depression, and anger) identified by Mitchell (2007), was hypothesized to have a direct effect on PTG. The single mediation path in this SEM was originally represented in RCOPE, which consisted of two indicators as expressed in the subscales of the Brief RCOPE (Pargament et al., 2011): PRC and NRC styles. However, in our sample, NRC did not contribute to spiritual coping and was removed from the present model. The hypothesized model thus configured was evaluated using IBM SPSS Amos and the results are presented in Figure 7.

The Chi-square for the model was found to be statistically significant at a value of $63.276(33, N=113), p=.001$, suggesting that the configured model was not a good fit to the data. Using a target value of $<0.95$ to identify an acceptable fit, the Normed Fit Index (.744) and Comparative Fit Index (.850) did not meet the criteria for a good fit. This was 
supported by the value of the Root Mean Square Error of Approximation (.091 with a $90 \%$ confidence interval of .056 to .124 ) which had a target value of $<.08$.

\section{Figure 7}

Hypothesized Path Model Relating Distress, Positive Spiritual Coping, and Posttraumatic Growth

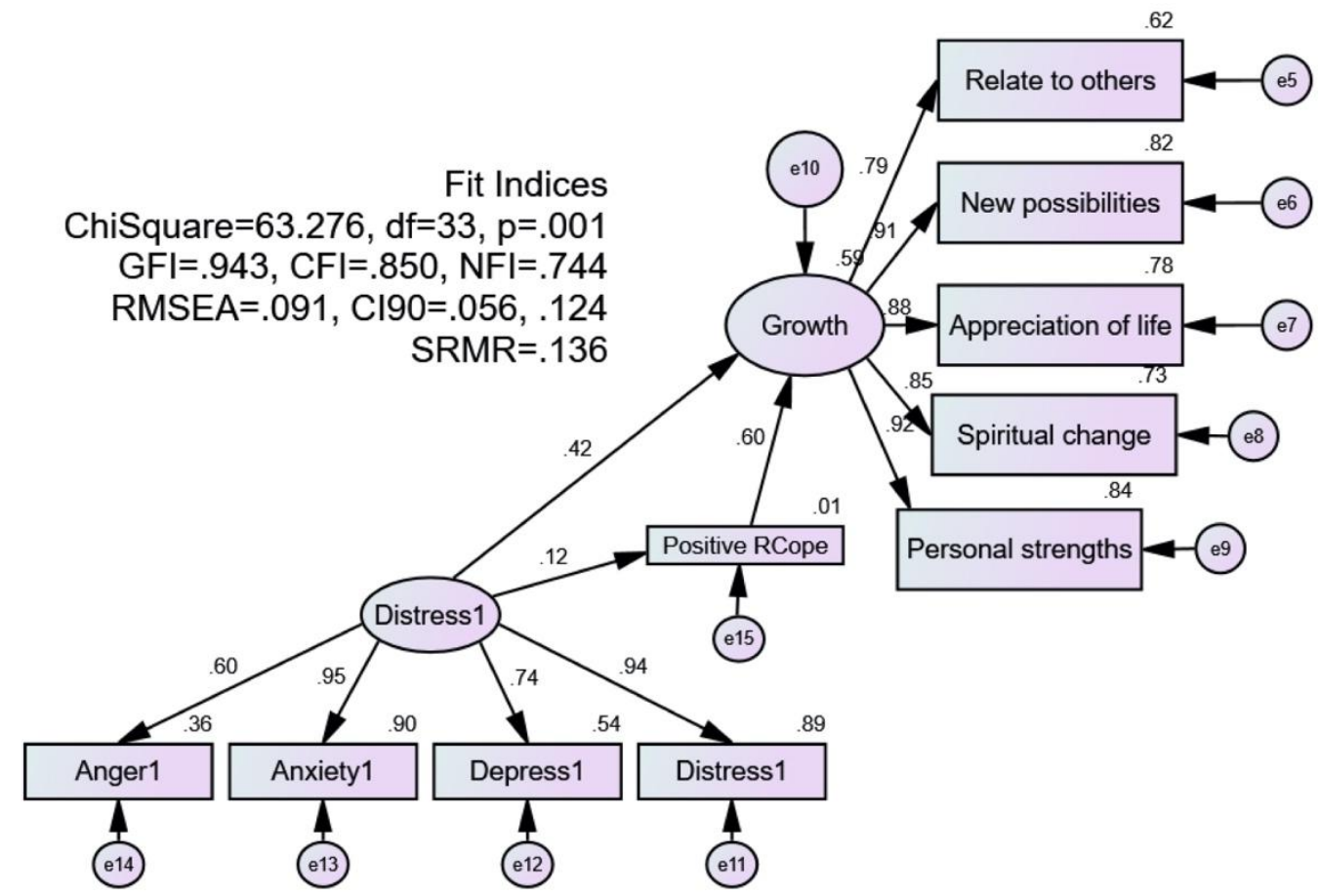

Modification indices were examined to determine where correlations of errors might be added to improve model fit. Using these indices, the error pair associated with distress and personal strength were identified and then correlated in the modified model. This study proposed that, as survivors seek to find relief in the wake of their abuse, distress drives the cognitive processes that are manifested first as intrusive rumination, and later as intentional cognitive engagement that leads to challenging past assumptions about others, self, and the world. In light of the extraordinary challenge they face, it makes sense that survivors will tap into internal resources and develop increased personal 
strength. Therefore, it makes sense that distress can lead to a perception of greater personal strength.

The results of the re-specified model are shown in Figure 8. The Chi-square in this analysis yielded a value of $54.896(32, N=113)$ and retained statistical significance $(p=.007)$. However, Chi-square divided by degrees of freedom was under 2.0 $(\mathrm{CMIN} / \mathrm{DF}=1.716)$. The Goodness of Fit Index (.95) and the Root Mean Square Error Approximation (.080, with a $90 \%$ confidence interval of .042 to .115) suggest a good fit, while other fit indices suggest a less than acceptable fit (Comparative Fit Index $=.89$; Normed Fit Index $=.78$; Standard Root Mean Square Residual $=.12$ ) See Table 16 for summaries of the initial and re-specified models.

\section{Figure 8}

Re-Specified Path Model Relating Distress, Positive Spiritual Coping and Posttraumatic Growth

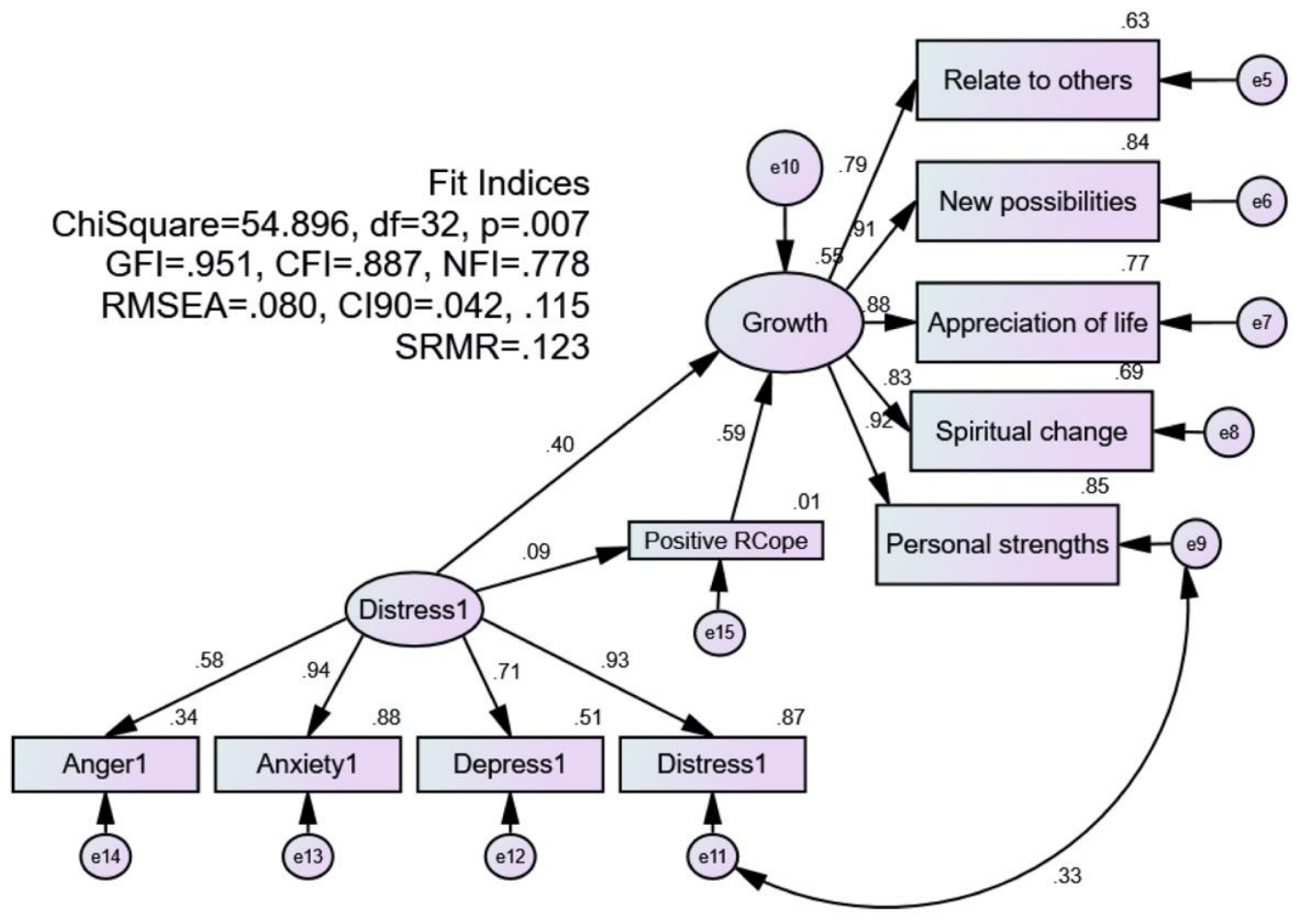


Table 16

Model Summaries for Distress, RCOPE, and PTG

\begin{tabular}{lrrrrrrrr}
\hline & CMIN & GFI & NFI & CFI & RMSEA & SRMR & N & df \\
\hline Model 1 Initial Model & 63.28 & .943 & .744 & .850 & .091 & .136 & 113 & 33 \\
Model 2 Re-Specified Model & 54.90 & .951 & .778 & .887 & .080 & .123 & 113 & 32 \\
\hline Target Values & & $<.95$ & $>.95$ & $>.95$ & $<.08$ & $0-.08$ & &
\end{tabular}

While the re-specified model did not appear to be an acceptable fit, it nevertheless accounted for 55\% of the variance in PTG in female survivors of SARL. An inspection of the path coefficients, presented in Table 17 , revealed that both the $\operatorname{PRC}(\beta=.597, \mathrm{p}=$ $<.001)$ and distress $(\beta=.416, \mathrm{p}=<.001)$ were significant predictors of PTG in this model. However, there was no correlation between distress and PRC $(\beta=.120, p=0.065)$ thus precluding a mediation effect of PRC on PTG. This suggests that PRC and Distress both contribute to PTG independently of one another.

Table 17

Path Coefficients Between Distress, RCOPE, and PTG (Re-Specified Model)

\begin{tabular}{lcrccccc}
\hline Path & & & $\mathrm{b}$ & $\mathrm{SE}$ & $\beta$ & \multicolumn{1}{c}{ CR } & \multicolumn{1}{c}{$\mathrm{p}$} \\
\hline PosRCope & $<---$ & Distress1 & 0.054 & 0.041 & .088 & 1.331 & 0.183 \\
Growth & $<---$ & Distress1 & 0.295 & 0.050 & .402 & 5.855 & $<.001$ \\
Growth & $<---$ & PosRCope & 0.707 & 0.093 & .590 & 7.568 & $<.001$ \\
Relate & $<---$ & Growth & 1.000 & & .793 & & $<.001$ \\
NewPossib & $<---$ & Growth & 1.171 & 0.069 & .914 & 17.062 & \\
AppreciateLife & $<---$ & Growth & 1.249 & 0.093 & .879 & 13.496 & $<.001$ \\
SpiritChange & $<---$ & Growth & 1.328 & 0.103 & .832 & 12.928 & $<.001$ \\
Q22Distress_1 & $<---$ & Distress1 & 1.000 & & .930 & & $<.001$ \\
Q22Depress_1 & $<---$ & Distress1 & 1.202 & 0.142 & .715 & 8.475 & $<.001$ \\
Q22Anxiety_1 & $<---$ & Distress1 & 1.036 & 0.056 & .936 & 18.505 & \\
Q22Anger_1 & $<---$ & Distress1 & 1.099 & 0.129 & .582 & 8.536 & $<.001$ \\
PerStrength & $<---$ & Growth & 1.160 & 0.067 & .925 & 17.319 & $<.001$ \\
\hline
\end{tabular}

The indirect effects of the mediation path from distress to coping (standardized coefficient $=.000$, standard error $=.038)$ and from coping to growth (standardized 
coefficient $=.000$, standard error $=.093$ ) had no practical significance. The direct path from distress to coping (standardized coefficient $=.088$ ) also had no practical significance. However, the direct paths from Distress to Growth (standardized coefficient $=.402)$ and from coping to growth (standardized coefficient $=.590)$ yielded significant results. For total effects from distress to growth the path coefficient was .454 , and from coping to growth it was .590 . These values confirm that positive religious/spiritual coping does not mediate the relationship between distress and PTG in this sample of survivors. However, distress and spiritual coping do appear to have a significant direct impact on PTG in this group. This study hypothesized that survivors with high levels of distress and PRC would experience higher levels of PTG than survivors with high levels of distress and NRC. These results support the stated hypothesis, although PRC and distress contribute independently to PTG and are not related to one another. The path coefficients are summarized in Table 18.

\section{Table 18}

\section{Direct and Indirect Effects of the Hypothesized Model}

\begin{tabular}{llccc} 
& & \multicolumn{3}{c}{ Causal Effects } \\
\cline { 3 - 5 } Outcome & Determinant & Direct & Indirect & Total \\
\hline Coping & Distress & .088 & .000 & .088 \\
& & & & .052 \\
Growth & Distress & .402 & .454 \\
& Coping & .590 & .000 & .590 \\
\hline
\end{tabular}

\section{Summary of Findings}

Based on a review of the literature, this study asked the following four questions:

(1) What was the context and nature of the SARL in our sample of female survivors? (2) What are the effects of spiritual coping on PTG among female survivors of SARL? (3) 
What are the effects of distress on PTG among female survivors of SARL? And (4) To what extent does spiritual coping mediate the relationship between distress and PTG among female survivors of SARL?

In response to Question 1, this analysis found that for a majority of the respondents the abuse was perpetrated by a Pastor/Priest/Bishop/Rabbi (90.2\%) over a period of time (92\%), occurred between the ages of 27 and 59 years old (61\%), occurred at least six times or more (73.4\%), and included a wide variety of sexual acts. Regarding Question 2, the analysis found that positive religious/spiritual coping $(\beta=.403, \mathrm{p}<.001)$ is a significant predictor of PTG. Our analysis of Question 3 found that the distress ( $\beta$ $=.416, \mathrm{p}<.001)$ experienced by survivors soon after the abuse, as recalled by participants, significantly predicted PTG in our sample. For Question 4, our study did not find that spiritual coping mediated the relationship between distress and PTG, as there was no relationship between distress and spiritual coping $(\beta=.088, \mathrm{p}=0.183)$. However, the direct effects of distress $(\beta=.402, \mathrm{p}<.001)$ and spiritual coping $(\beta=.590, \mathrm{p}<.001)$ on growth was confirmed in this analysis. 


\section{CHAPTER 5}

\section{SUMMARY, DISCUSSION, AND RECOMMENDATIONS}

\section{Introduction}

This chapter summarizes and discusses the major findings of this study. It begins with a review of the problem, the purpose of the study, and its significance to the current body of research. An abbreviated summary of the literature and the methodology used by the researcher follows. A major portion of this chapter is dedicated to an extensive discussion of the results in view of the literature presented. Finally, I identify the limitations of the study and make recommendations for practice and future research.

\section{Research Problem}

While news of sexual abuse by trusted religious leaders continues to emerge at various times and diverse locations, it is not a new or recent phenomenon. In the past 20 years, the impact of this abuse has become the subject of ongoing discourse and study, even as it becomes a heartbreakingly familiar topic. The psychological, emotional, and spiritual fallout of these experiences linger in the survivors for many years beyond the

time of the event(s) (e.g., Benkert \& Doyle, 2009; Doyle, 2009; Farrell, 2009; Pargament, Murray-Swank et al., 2008; Shea, 2008). For survivors, the distinctive nature of the abuse lies in the fact that their abuser was a respected and highly revered individual within a trusted religious system. Many perceived their abuser as very close to God. The 
psychological preparation (i.e., the grooming process) increased the victim's vulnerability to the abuse and created a toxic spirituality that determined the shape of the abuse (Doyle, 2006). Adding to the complexity of the traumatic experience is the apparent lack of redress the victims have received from their religious organizations after disclosing the abuse (Fater \& Mullaney, 2000; Flynn, 2008; McMackin et al., 2008).

This study assumed that survivors, as members of a spiritual community, experienced a form of toxic spirituality that created the environment which enabled their abuse. In spite of this, the study contended that the presence of spiritual coping would mediate the path from distress to PTG and would increase PTG in these survivors. This study also contended that positive spiritual coping styles would yield the greatest levels of growth.

\section{Purpose of the Study}

The overall goal of this study was to explore the mediatory role of spiritual coping styles on the relationship between distress and PTG in female survivors SARL. Other goals of this study included exploring the context in which survivors experienced the abuse, an examination of the relationship between distress and PTG, and an examination of the relationship between spiritual coping and PTG.

This study sought to identify the impact of positive and negative spiritual coping styles on the outcome of PTG in a very specific population by examining the direct and indirect effects of distress and spiritual coping on PTG, and by determining whether a mediatory effect exists. 


\section{Significance of the Study}

This study contributes to the empirical literature by providing additional evidence for the notion that psychological benefits can occur in the wake of highly distressing events. Support for this idea has been documented among many specific populations and for at least some individuals (e.g., survivors of childhood sexual abuse, adult sexual assault, and cancer). This study not only adds to the body of literature on this topic but specifically contributes to a greater understanding of PTG in female survivors of SARL.

This study also contributes to the current knowledge base that attempts to understand the relationship between distress and PTG. The present research on this relationship has yielded mixed results, with many studies suggesting that the relationship between distress and PTG is positive, while others find no relationship or a negative relationship.

Predictive factors in PTG have been identified by some of the current literature. For example, research suggests that the presence of cognitive engagement and eventrelated rumination are predictive of PTG (Calhoun \& Tedeschi, 2006; Calhoun, Cann et al., 2000; Cann, Calhoun, \& Tedeschi et al., 2011). This study adds to the literature by providing some insight into the role of spiritual coping as a predictive factor in PTG among survivors of sexual abuse.

Another important contribution of this study involved an exploration of the types

of spiritual coping that contributed to PTG. While there is evidence in the literature to suggest that positive spiritual coping is related to growth and resilience in trauma survivors (Shaw et al., 2005; Pargament, 1997), this study added information to the knowledge base regarding how this holds true for survivors of SARL. 


\section{Summary of the Literature}

In the past 30 years, the well-worn notion of growth through suffering has been the subject of inquiry for a select group of researchers. The idea that growth can result from debilitating life crises is not a novel one. It has been explored in literature, art, and folklore. Recently, it has been explored through scientific inquiry with many interesting results. Various labels for this type of growth have appeared in the literature, with subtle differences in meaning. Some examples of these include "stress-related growth" (Park et al., 1996), “illusory growth” (Hobfoll et al., 2007), “thriving” (O’Leary and Ickovics, 1995), and "posttraumatic growth" (Tedeschi \& Calhoun, 1996, 2004b).

This study focused on PTG defined as, "the positive psychological change experienced as a result of the struggle with highly challenging life circumstances" (Tedeschi \& Calhoun, 1996, p. 1). In this model, PTG is determined by growth that "surpasses" the previous functioning in one or more of the domain areas identified by

their research: (1) enhanced relationships, (2) the perception of new life possibilities, (3) increases in gratitude and valuing of life, (4) a new awareness of competence/capability, and (5) an awakened or enhanced spirituality. This study explored PTG in relationship to distress and spiritual coping in a sample of adult female survivors of SARL, as well as the mediatory effects of spiritual coping on PTG.

Tragedy and debilitating life experiences have frequently been associated with psychological disorders in the literature. However, a far greater number of trauma survivors report experiences of positive growth in the aftermath of adversity and potentially traumatic events (Tedeschi \& Calhoun, 2004b). This does not suggest that 
growth will always occur, nor that distress related to the traumatic events is absent. Instead, it appears that growth frequently co-exists along with continued distress.

According to the PTG model, life crises cause assumptions about the world to be shaken or shattered, destabilizing foundational assumptions. Survivors try to make sense of difficult events that no longer fit neatly into existing life schemas or previously held worldviews. This cognitive dissonance challenges existing schemas, giving rise to persistent event-related rumination—first intrusive and later intentional—as survivors work to reduce distress by attempting to make sense of disturbing events (Calhoun \& Tedeschi, 2006; Taku et al., 2009). The model contends that when a person experiences a seismic life event, emotional distress causes dysregulation; challenges beliefs about the world, self, others; challenges life narratives. This leads to intrusive rumination and then to behaviors and activities that seek to relieve distress, such as talking about the experience, writing, self-disclosure, and spiritual coping. Eventually, rumination becomes a form of cognitive engagement that is more intentional and searching. At this point, life narratives, schemas, spirituality, and/or goals may be modified or changed, often in positive ways. Higher levels of engaged rumination following a traumatic event seem to create fertile ground for growth (Helgeson et al., 2006; Tedeschi et al., 2007). This study sought to explore whether spiritual coping would become a useful resource at the stage of engaged rumination for increasing the PTG potential in survivors.

Spiritual resources have been associated with growth in many adult survivors of sexual abuse who are also experiencing high levels of distress (Walker-Williams et al., 2013). Positive coping behaviors identified by survivors included spiritual coping strategies and a renewed personal relationship with God. Challenges to personal 
narratives resulted in psychological changes that lead to a reformulated or reconstructed life schema. Thus, spirituality has been identified as a predictor of PTG (Cadell et al., 2003). Other identified predictors include supportive relationships (Dursun et al., 2016; Fetty 2016), meaning in life (Grad \& Zeligman, 2017; Dursun et al., 2016), cognitive processing and event-related rumination (Tedeschi et al., 2007; Helgeson et al., 2006), and disclosure and life narrative (Neimeyer, 2004; Neimeyer et al., 2006).

Spirituality and religion appear to provide some survivors of sexual abuse with a powerful impetus to make sense of their tragedy (Ganzevoort, 2001). Studies have also linked religion to greater well-being (Kennedy et al., 1998), a source of sanity-increasing resilience (Israel-Cohen, et al., 2016; Chen \& Koenig, 2006), and recovery (Linley \& Joseph, 2004; Shaw et al., 2005) in survivors of sexual assault. However, negative and positive religious coping patterns appear to be related to different outcomes (Pargament et al., 2000). A meta-analysis by Ano and Vasoconcelles (2005) found that positive psychological adjustment was related to positive religious coping patterns, while survivors who engaged in negative religious coping experienced higher levels of maladaptive adjustment.

PTG has also been linked to psychological distress (Cann et al., 2010; Grubaugh $\&$ Resick, 2007). However, much of the research on the relationship between psychological distress and PTG has yielded mixed results, with some suggesting a positive relationship and others finding no relationship (e.g., Chopko, 2010; Helgeson et al., 2006; Laufer \& Solomon, 2006; Tedeschi \& Calhoun, 2004; Zoellner \& Maercker, 2006). However, this study found considerable evidence to suggest that psychological 
distress can create an environment for potential growth according to the Calhoun and Tedeschi (2006) model of PTG (Chopko, 2010; Tedeschi \& Calhoun, 2004a).

Thus, the intention of this study was to further explore the relationships between PTG, distress, and spiritual coping based on relationships presented in past research for these variables. This study sought to examine the mediatory role of spiritual coping in the relationship between distress and PTG. The goal of this study was to explore these relationships within a sample of female survivors of SARL.

\section{Methodology}

The present study sought to answer the following questions in a sample of female survivors of SARL: (1) What was the context and nature of the abuse? (2) What were the effects of spiritual coping on PTG? (3) What were the effects of distress on PTG? (4) To what extent does spiritual coping mediate PTG in this population?

The study was designed as a quantitative, correlational, non-experimental design using validated self-report measures and survey data. Data was collected via SurveyMonkey, a secure online survey software. The relationships between the variables (i.e., distress, spiritual coping, and PTG) were evaluated using the multiple regression methodology of path analysis and structural equation modeling (SEM). PTG was measured using the PTGI, spiritual coping was measured with the Brief RCOPE, and distress was measured via the ET (see Appendix E). Demographic data was gathered using the Demographic Data Sheet (see Appendix C), while specifics about the circumstances of the abuse were collected using the Context of Abuse Questionnaire (see Appendix D). 
Participants in the sample were recruited from among the members of THOS, a U.S. national non-profit organization set up to provide support and counseling to women who have experienced SARL. Surveys were sent electronically to all the members of THOS database which has several thousand individuals. Individuals included in the study had to (1) be female, (2) be 18 years or older, and (3) have had at least one self-identified experience of sexual abuse/assault by a religious leader. A total of 113 of the returned responses were usable and retained for this study.

\section{Demographic Characteristics of the Respondents}

A total of 143 participants responded to the survey. However, 30 questionnaires were excluded from the analysis due to incomplete data (i.e., a minimum of $10 \%$ missing responses). Thus, a total of 113 respondents met full criteria for inclusion and made up the final sample for this analysis. All of the participants were members of THOS, with $83 \%$ reporting that they had been members for at least six months at the time of the study. The sample was made up of predominantly White participants $(81.4 \%)$ with Black participants making up $10.6 \%$ of the sample, while Latino (1.8\%), bi-racial (4.4\%), Asian $(.9 \%)$, and Caribbean $(.9 \%)$ participants made up the remaining $8 \%$ of the sample. The mean age was 47.4 years old, with a standard deviation of 12.5 years and a range of 20 to 76 years; $68 \%$ of the sample ranged in age from 35 to 70 years. A majority of the sample held a bachelor's degree or higher (63.7\%) and identified with a Christian faith group (71.7\%). Participants who no longer identified with any organized religion $(9.7 \%)$ or claimed to have drastically changed their faith practices $(3.5 \%)$ since their experience of abuse made up $13.2 \%$ of the respondents. A total of $84.9 \%$ identified themselves as being more or very religious. 


\section{Summary and Discussion of Major Findings}

\section{Research Question 1}

What was the context and nature of the SARL in our sample of female survivors? The purpose of this question was to obtain a picture of the nature and circumstances of the abuse.

\section{Results}

The survey in our sample found that $92.1 \%$ of survivors experienced the abuse in adulthood (61.1\% between 27 and 59 years old; $31 \%$ between 19 and 26 years old). A quarter of survivors $(24.8 \%)$ reported that the abuse had occurred during adolescence (11 to 18 years old), while $6.2 \%$ reported that it occurred during childhood (4 to 10 years old). Many reported that their abuse experience continued over a period of years, which may explain the high percentage totals for this question.

A majority of the participants in this study $(73 \%)$ reported that the abuse had occurred at least six times, with $50 \%$ of the total reporting that it had occurred more times than they could remember. Ninety-two percent of respondents stated that their abuse had occurred over time, with $41 \%$ stating that it continued for three or more years. Approximately 90\% identified their abuser as a Pastor, Priest, Bishop, or Rabbi; 19\% indicated that their abuser was an Elder or Deacon; $8 \%$ identified the abuser as other religious leaders (e.g., Seminary Professor, Navy Chaplain, Teacher, Pathfinder Leader).

A majority of the respondents $(63 \%)$ reported seeking and receiving counseling in the two years following the abuse, with $26 \%$ stating they received counseling for two or more years. Approximately $9 \%$ of respondents received counseling for one to two years, and $30 \%$ received counseling for one year or less. 
Regarding the nature of the abuse, respondents reported a wide variety of sexual acts. A majority of respondents reported experiencing fondling or touching to evoke sexual feelings (82.3\%); sexually explicit, suggestive, or humiliating comments/language (71.7\%); and penetrative sexual acts $(56.6 \%)$. Between $29 \%$ and $40 \%$ of respondents reported experiencing coercion or encouragement to masturbate, exposure to masturbation or other sexual acts, and indecent exposure. A smaller percentage reported being exposed to pornography $(12.4 \%)$ or being coerced to create pornographic images or videos $(18.6 \%)$.

In summary, while each survivor's experience was unique, for the typical female survivor in this sample, the context of abuse included repetitive sexual harassment or coercive acts that occurred over period of six months to over five years and was perpetrated by a prominent religious leader upon a female adult member of their congregation. Many of these survivors sought counseling shortly after the abuse.

\section{Discussion}

Much of the discussion in the research around SARL has focused on child victims, particularly male children. This study explored the experience of adult female women for whom there appears to be much less data. Approximately $31 \%$ of the sample indicated that their experience had occurred during childhood $(6.2 \%)$ or adolescence $(24.8 \%)$, while the majority reported that it occurred in adulthood $(92 \%)$. There is a common misconception that the sexual abuse of adult women by religious leaders is nothing more than a discussion about consensual affairs. However, a description of the nature and context of the abuse, as depicted by the participants in this sample, tells a different story. Ninety percent of survivors described sexual abuse by a Pastor, Priest, or 
Rabbi - the head of a body of believers. The violation that occurs here affects survivors at multiple levels: the violation of the sacred role of spiritual leader, the desecration of the relationship between the believer and the leader, the violation of a system that legitimized the offender, and a violation of the most vulnerable parts of human identity. The various layers of this violation have been discussed in the literature (Pargament et al., 2008).

In our sample, $65 \%$ of survivors sought counseling in the two years following the abuse, suggesting that survivors were struggling with the psychological and emotional fallout of the experience. Survivors of SARL have been known to struggle with issues of self-worth, interpersonal relationships, emotional regulation and expression, job performance, and home life, etc. (Fater \& Mullaney, 2000). Many participants in our sample were previously members of a religious or spiritual group, thus it is likely that they also encountered spiritual struggles as a result of their experience. Pargament et al. (2008) have found that spiritual struggle is often the result of adversity. This struggle may manifest as anger toward God for not protecting the survivor, anger toward a religious or spiritual institution/system that failed to respond appropriately, conflict with family members or church members, as well as internal conflicts and uncertainly regarding personal beliefs (Pargament, Magyar-Russel et al., 2005). It was apparent that some survivors in this study struggled with retaining their spiritual beliefs and practices and others abandoned previously held practices altogether. This is consistent with research suggesting that the experience of sexual abuse has caused survivors to distance themselves from spiritual practices that they once held close (Walker et al., 2009). However, survivors who are able to preserve some form of spiritual coping have been shown to develop fewer trauma symptoms than those who had no spiritual coping 
practices (Walker, Reid, O’Neill, and Brown, 2009). For the sample in this study, it is likely that spiritual struggle played a role in the outcome of PTG. This is addressed in the discussion for Question 2.

\section{Research Question 2}

What are the effects of spiritual coping on PTG among female survivors of

\section{SARL?}

\section{Hypothesis}

Spiritual coping will directly affect the level of PTG in female survivors of SARL and survivors with positive spiritual coping styles will experience higher levels of PTG than those who used negative spiritual coping styles.

\section{Results}

The path analysis explored the predictive ability of PRC and NRC styles in the five domain areas of PTG: relating to others, new possibilities, personal strength, spiritual change/growth, and appreciation of life.

The results of this analysis found that spiritual coping was a significant predictor of PTG, with a standardized regression weight of .574 . Spiritual coping accounted for $33 \%$ of the variance in PTG in our sample. However, the investigation found that the variance in PTG was primarily due to the direct effects of PRC (path coefficient for PRC $=.70$ ) rather than $\mathrm{NRC}$ (path coefficient for $\mathrm{NRC}=.07$ ). This outcome indicates that PRC, but not NRC, significantly contributes to PTG, confirming the hypothesis for Question 2.

In the PRC, seeking God's love and care (70\%), searching for a stronger connection with God (69\%), and asking forgiveness for sins (65\%) were among the most 
highly endorsed statements overall. At least half of the participants attempted to include God in their plans (58\%), searched for ways in which God was trying to strengthen them $(58 \%)$, and sought to let go of anger $(50 \%)$. The analysis additionally found that a direct

correlation existed between spiritual/religious coping style — specifically, $\mathrm{PRC}$ - and the spiritual change/growth subscale of the PTGI. Thus, not surprisingly, positive spiritual coping was directly related to the aspect of PTG that measures spiritual change and spiritual growth.

\section{Discussion}

Spiritual struggle can lead to positive changes and growth in spirituality or it can lead to disengagement from previously held beliefs (Walker et al., 2009). In our sample, respondents appeared to use NRC almost as frequently as PRC. For example, the following item on the NRC was endorsed more than all the other items on the Brief RCOPE: Wondered whether my church had abandoned me. A total of $78 \%$ of respondents agreed with this statement quite a bit or a great deal. This indicates that the majority of survivors in our sample felt abandoned by their spiritual communities upon disclosure of the abuse. In addition, over half of respondents (57\%) wondered whether they had been abandoned by God himself. These negative thoughts are evidence of a spiritual struggle, which can be a precursor to rejecting spirituality in part or as a whole. Thus, a framework that was once a useful resource in facing adversity for this population is no longer an option they can rely on. Spiritual struggle can lead to negative coping (e.g., wondering whether God or their church had abandoned them), which may create a psychological environment that inhibits growth in many survivors. In our sample, this may explain the lack of relationship between NRC and PTG. 
The present study found a clear contrast between the use of negative and positive coping styles in our sample, in relationship to PTG. PRC significantly predicted PTG. There is evidence to suggest that individuals who claim to have no spiritual or religious affiliations experienced higher levels of traumatic symptoms than those who practiced some form of spirituality or religion (Walker et al., 2009). Certain spiritual practices appear to act as protective factors in the face of adversity and the absence of these, particularly for individuals who once identified as religious or spiritual, appears to increase their vulnerability to traumatic symptoms. The results of this study offer evidence that, beyond the potential for increased resilience against traumatic symptoms, PRC styles create a favorable environment for PTG in the wake of SARL. While not all research on the impact of spiritual coping distinguishes between positive and negative coping styles (e.g., the meta-analysis by Walker et al., 2009), this study supported the distinction between these two styles of spiritual coping.

The statements most commonly endorsed by respondents from the PRC scale were those that included a seeking of God's love and care, asking forgiveness for sins, attempting to include God in their plans, searching for ways that God might be trying to strengthen them, and seeking to let go of anger. These practices suggest a secure connection with God or Higher power, which allowed survivors to turn to their spirituality in the midst of a crisis, even while life narratives and spiritual meaning were being challenged. Challenges to spiritual meaning created the conditions for growth, as survivors worked to restructure their narratives in order to accommodate the new information. When assumptions about the world are shattered, the resulting destabilization can lead to changes in spirituality. Statements that reflected a secure 
relationship with God (or Higher Power) help set the stage for the meaning-making process that appears to be an essential component of growth after trauma (Vilencia et al., 2013). When survivors use thought patterns that reflect this secure connection, they are more likely to experience growth.

\section{Research Question 3}

What are the effects of distress on PTG among female survivors of SARL? Participants in this study were asked to report on the level of distress they experienced shortly after the abuse took place, to the best of their recollection.

\section{Hypothesis}

Distress will be positively associated with PTG and survivors with high levels of distress will experience greater PTG than those with low levels of distress.

\section{Results}

In this sample, the reported distress levels were exceptionally high and the distribution was extremely negatively skewed. All of the four subscales in the distress measure (ET) had a median of 10 out of 10, and the means ranged from 7.80 to 9.20 (out of 10) indicating that this sample of survivors experienced very high levels of distress. This is probably due to the retrospective style in which the questions were asked. Participants were asked to recall their distress in the period shortly after the abuse took place. Thus, emotional and psychological distress (i.e., anxiety, anger, depression, distress) in the immediate aftermath of the abuse was likely to be at its height. Due to the levels of distress in this sample, it was not possible to evaluate the effects of mild or moderate distress on PTG as most of the participants were in the very high range. 
The path analysis in this study explored the direct path from distress to PTG and found that high distress was a significant predictor of PTG in our sample. Distress accounted for $17 \%$ of the variance in PTG reported by survivors of SARL with a standardized regression weight of .416 .

\section{Discussion}

This positive relationship between distress and PTG may be explained by the power of distress to spur survivors toward relief-seeking behaviors. This search for relief is the driving force behind the cognitive engagement that begins as intrusive rumination, and later becomes intentional rumination (or cognitive processing), which leads to eventual growth (Tedeschi \& Calhoun, 2004a).

Survivors of trauma frequently experience the automatic, intrusive reliving of painful events in the aftermath of trauma. This often moves into more intentional cognitive engagement as survivors attempt to process and make sense of the events. Driven by persistent distress, many survivors of SARL find themselves adjusting and restructuring personal narratives into stories that are better able to accommodate the new information stemming from the trauma. At this point, meaning making appears to be an essential aspect of the growth journey for survivors of SARL. This process has been supported by research (Grad \& Zeligman, 2017; Neimeyer et al., 2006; Vilencia et al., 2013). However, while meaning making and the restructuring of life narratives may lead to growth in many cases, it is not a guaranteed path. PTG is not an inevitable outcome of trauma.

In this analysis, a positive link between distress and PTG was observed. This suggested that the presence of psychological distress may play a role in providing an 
ideal cognitive environment for PTG. Thus, higher levels of distress seemed to prompt survivors of SARL to engage in the cognitive processes that drive changes in narrative, long-term goals, and even in spiritual beliefs. Survivors who sought relief through actions such as seeking a closer connection with God/Higher Power, seeking God's love and care, releasing anger, self-disclosure, and support-seeking seemed to increase their opportunities for developing alternative narratives, and thereby increase PTG. This process is supported by the literature on PTG (Tedeschi \& Calhoun, 1996, 2004b; Neimeyer et al., 2006). In our sample, a majority of respondents (81\%) agreed that they have more compassion for others since the abuse. This seems to suggest that there was an increase in psychological and/or emotional connectedness to others after the event, which appeared to help facilitate growth in the area of interpersonal relationships. Some studies suggest that traumatic events create an environment suited for growth due to the severe psychological challenges that arise in the aftermath of trauma (Cann et al., 2010; Chopko, 2010; Grubaugh \& Resick, 2007), while others find these relationships to be unreliable (Tedeschi and Calhoun, 2004b; Zoellner \& Maercker, 2006). Nevertheless, this study supported the body of research that suggests there is a positive relationship between these two variables.

\section{Research Question 4}

To what extent does spiritual coping mediate the relationship between distress and PTG among female survivors of SARL? This question explored the mediatory role of spiritual coping between these variables. 


\section{Hypothesis 3}

Survivors with high levels of distress and PRC will experience higher levels of PTG than women with high levels of distress practicing NRC.

\section{Results}

The relationship between the variables was explored using path analysis and SEM. The overall values of our mediation model found that spiritual coping did not sufficiently mediate the PTG outcome to be considered significant. The analysis found that there was no relationship between distress and spiritual coping in our sample. Thus, spiritual coping did not significantly affect the relationship between distress and PTG among female survivors of SARL.

\section{Discussion}

The results of this study found that, as a consequence of the non-significant relationship between spiritual coping and distress, spiritual coping was unable to influence the relationship between distress and PTG. This lack of association between distress and spirituality appears to be inconsistent with literature suggesting that, in times of traumatic distress resulting from sexual assault or other life crises, many spiritual individuals turn to spiritual and religious activities as a source of strength and answers (Kennedy et al., 1998). The absence of a relationship between these two variables in our sample may reflect an inherent contradiction in the experience of survivors of SARL. While survivors who identified as spiritual might have relied on spiritual resources in difficult times, survivors of SARL may be reluctant to turn toward a resource that now represents an unsafe system, and where they have experienced injury. The spiritual self is 
one of the numerous areas of the self-identity that sustains negative effects as a result of sexual abuse perpetrated by religious leaders (Easton et al., 2019; Huson, 2002).

In this research, PRC and NRC styles were measured in relationship to high, medium, or low levels of distress. Virtually all participants reported experiencing high distress levels shortly after the abuse. And while participants reported using NRC and PRC strategies almost equally, only positive spiritual coping was related to growth. This supports previous research suggesting that PRC practices offer psychological benefits to the believer (Ano \& Vasconcelles, 2005; Pargament, 1997). Distress was not found to be related to positive or negative spiritual coping, suggesting that distress did not increase or decrease the amount of positive or negative spiritual coping in which participants engaged. This does agree with the literature which suggests that survivors who are spiritual frequently turn to their spiritual frameworks and worldviews for answers (Pargament, 1997; Pargament et al., 2000; Pargament \& Raiya, 2007). Personal beliefs are often acutely challenged in the aftermath of trauma as many struggle with current spiritual frameworks (Tedeschi \& Calhoun, 2006). However, in this study, distress was measured in retrospect (i.e., based on participants' memory shortly after the abuse occurred), while spiritual coping was measured in current time (i.e., at the time of the survey). Thus, these results suggest that the distress survivors experienced shortly after the abuse is not related to the spiritual coping strategies in which they engage currently. It does not speak to the ways in which distress may or may not be related to spiritual coping immediately following the abuse. This may explain the absence of a link between the two variables. 
The lack of association between distress and spiritual coping in this sample may also be due to the very high levels of overall distress reported by survivors. The disclosed levels of distress revealed little variation, most being in the high and very high range. This lack of variation would likely render any association between distress and spiritual coping indiscernible.

Nevertheless, our investigation found that the presence of religious/spiritual coping did not significantly alter the impact of distress on PTG in our sample. While the indirect effects in the mediation model between the three variables were not significant, the significant relationship of the direct effects of distress on PTG and spiritual coping on PTG was confirmed in this analysis. Spiritual coping had a direct impact on PTG, as did distress. This supports previous research that suggests both spiritual coping and distress can increase PTG (Cann et al., 2010; Chopko, 2010; Grubaugh \& Resick, 2007; Walker et al., 2009; Vilencia et al., 2013). When both distress and spiritual coping were present, PTG increased, not due to a mediation effect, but because both contributed to PTG independently and appeared to have a cumulative impact on PTG. The fact that there was no relationship between distress and spiritual coping in our sample precluded a mediation effect.

\section{Limitations of the Study}

This study had several limitations. The population sample was recruited from a single organization whose members are predominantly of Christian faith backgrounds and may not adequately represent individuals of non-Christian faith or spiritual groups. Additionally, survivors who no longer subscribe to any faith tradition or spirituality are 
not likely to be well represented in our sample, due to the nature of the organization from which the sample was drawn. This limited generalizability.

In addition, the present study used self-report measures to assess past distress, as well as spiritual coping styles and PTG. Self-report measures present specific difficulties that may result from the behavior and attitude of the respondents. These may include (1) exaggerated or minimized responses; (2) responses that are influenced by mood, energy level, or time of day; (3) responses biased for social desirability; (4) an attempt to appear more distressed than normal/usual; (5) a desire to confirm or disconfirm the researcher's hypothesis; and/or (6) inability to remember details relevant to the study. This list of difficulties/challenges of self-report measures is not comprehensive. The outcomes additionally reflect the results of surveys that were voluntarily returned by participants and may reflect a bias of self-selection.

Another limitation of this study was that it examined only responses from the perspective of female survivors of SARL. Therefore, it is limited in its application and generalizability to male survivors of SARL.

\section{Recommendations for Practice}

While this study found that spiritual coping did not mediate the relationship between distress and PTG, it did find that positive spiritual coping had a significant direct impact on PTG. This suggests that the coping styles associated with a secure relationship with God, a sense of spiritual connectedness with others, attributions of meaning to life, benevolent appraisals, forgiveness, and seeking spiritual/religious support are related to overall growth. This study then, lends support to the current literature suggesting that spiritual and religious practices are a resource to survivors in their journey toward healing 
and adaptive functioning. As such, clinical practice may benefit from tapping into such resources where they exist. Clinicians may also assist survivors in adjusting negative styles of coping to positive styles of coping, in order to encourage PTG.

This study additionally found that distress had a significant direct impact on PTG. This was consistent with some of the literature on PTG. In this study, distress was identified as the psychological and emotional pain that interferes with daily functioning and results in negative views of others, self, and the world—presumably as a result of SARL in female survivors. Distress leads to rumination (intrusive and intentional). Studies have suggested that specific types of rumination (i.e., cognitive processing and traumatic event-related rumination) are a precursor to growth (Greenberg, 1995; Helgeson et al., 2006; Tedeschi et al., 2007). In particular, the type of cognitive engagement that contributes to meaning making and the restructuring of life narratives appear to be an essential aspect of growth after trauma (Pals \& McAdams, 2004; Tedeschi \& Calhoun, 2004; Vilencia et al., 2013). With this understanding, it is recommended that clinicians assist survivors in moving toward intentional cognitive engagement that leads to meaning making and readjustments of life narratives in order to facilitate PTG. Nevertheless, it is essential to understand that distress may persist and may co-exist with growth.

\section{Recommendations for Future Research}

This study sought to explore the relationship between distress, spiritual coping, and PTG in a very specific population: female survivors of SARL. While the PTG has, and continues to be, a well-researched construct among numerous populations, research continues to be scarce in the population from which our sample was taken: survivors of 
SARL. These survivors represent unique struggles and contradictions that remain poorly understood. Additional studies can further explore spirituality and spiritual coping as a predictor of PTG in this population, which can lead to a greater understanding of the specific activities that promote growth. Further studies, including qualitative explorations, on the experience of these survivors, and the impact of heightened distress on spirituality will add valuable information to our knowledge base in this particular population.

The relationship between distress and spiritual coping in this study was not found to be significant. This may be due to the size of our sample. Or it may be due to the conflict that arises for survivors who must decide whether they will turn to a religious or spiritual body that they believe has caused them injury. Alternately, the results may be due to a lack of relationship in the larger population. Further qualitative and quantitative studies may offer insight into the dynamics of the relationship between distress and spiritual coping.

In this study, distress was found to directly predict PTG. With our current understanding of the mechanisms through which PTG and distress are linked, based on the extant literature, (i.e., cognitive processing or intentional rumination, meaning making, restructuring life narratives), further research regarding the clinical applications of specific therapeutic modalities that facilitate these changes would add tools to our evidence-based practice. 
APPENDIX A

APPROVAL LETTER 


\section{Andrews DUniversity}

June 20, 2019

Angela St. Hillaire

Tel: (269) 240-8915

Email: angelas@andrews.edu

RE: APPLICATION FOR APPROVAL OF RESEARCH INVOLVING HUMAN SUBJECTS

IRB Protocol \#: 19-044 Application Type: Original Dept.: Graduate Psychology \& Counseling

Review Category: Full Action Taken: Approved Advisor: Elvin Gabriel

Title: Spiritual coping as a mediator between distress and posttraumatic growth in adult female survivors of sexual abuse by religious leaders.

This letter is to advise you that the Institutional Review Board (IRB) has reviewed and approved your IRB application for research involving human subjects entitled: "Spiritual coping as a mediator between distress and posttraumatic growth in adult female survivors of sexual abuse by religious leaders" IRB protocol number 19-044 under Full category. This approval is valid until June 20, 2020. If your research is not completed by the end of this period you must apply for an extension at least four weeks prior to the expiration date. We ask that you inform IRB Office whenever you complete your research. Please reference the protocol number in future correspondence regarding this study.

Any future changes made to the study design and/or consent form require prior approval from the IRB before such changes can be implemented. To request for extension, modification and completion of your study please use the attached form.

While there appears to be no more than minimum risk with your study, should an incidence occur that results in a research-related adverse reaction and/or physical injury, this must be reported immediately in writing to the IRB. Any project-related physical injury must also be reported immediately to the University physician, Dr. Katherine, by calling (269) 473-2222.

We wish you success in your research project. Please feel free to contact our office if you have questions.

Sincerely,

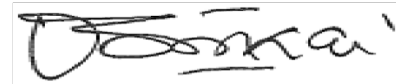

Mordekai Ongo, Ph.D.

Research Integrity \& Compliance Officer

Institutional Review Board - 8488 E Campus Circle Dr Room BUL 234 - Berrien Springs, MI 49104-0355 Tel: (269) 471-6361 Fax: (269) 471-6543 E-mail: irb@andrews.edu 
APPENDIX B

INFORMED CONSENT 


\section{INFORMED CONSENT}

\section{Purpose}

You are invited to participate in the present research study entitled SPIRITUAL COPING AS A MEDIATOR BETWEEN DISTRESS AND POSTTRAUMATIC GROWTH AMONG ADULT FEMALE SURVIVORS OF SEXUAL ABUSE BY RELIGIOUS LEADERS. The primary purpose of this study is to explore whether spiritual coping affects the relationship between distress and posttraumatic growth (PTG) in female survivors of sexual abuse by religious leaders. This study will examine the extent to which spiritual coping styles affect the outcome of posttraumatic growth in this population.

\section{Researchers}

This research is being conducted by Angela St. Hillaire, a PhD student in the Department of Graduate Psychology and Counseling at Andrews University in Berrien Springs, Michigan. Dr. Elvin Gabriel, EdD, is providing oversight for this research. Research findings will become part of Angela St. Hillaire's dissertation and may be used for publication in academic journals or presentations at conferences.

\section{Procedure}

If you chose to participate in this study, you be asked to complete an anonymous survey that includes questions about yourself, the nature and context of the sexual abuse, what spiritual coping strategies you used, the areas in which you experienced growth, and your past and current levels of distress. The survey will take approximately 20 to 25 minutes to complete.

\section{Participation}

To participate in this survey you must be 18 years of age or older, female, and a survivor of sexual abuse by a religious leader. Participation is completely voluntary. You may choose to participate, or not. Once you begin the survey, you may choose to discontinue at any time.

\section{Risks and Benefits}

Whenever there is discussion about past sexual abuse, there is the possibility that memories will arise that lead to fear, anxiety, or depression. In the event that an item on the survey provokes an intense reaction, please feel free to discontinue the survey and seek therapeutic support. Counseling and therapeutic support is generally provided by The Hope of Survivors (THOS) as part of their services to members. THOS has confirmed that special attention will be paid to making this support available during the survey period.

Alternately, the opportunity to tell one's story can have beneficial impacts, such as creating a sense of being set free, feeling heard, lifting depression, and reducing isolation. In addition, your participation in this study will contribute to the goal of gaining a better understanding of how posttraumatic growth is facilitated in survivors of sexual abuse by religious leaders. This understanding can help guide women who have suffered the same abuse toward growth and healing.

\section{Confidentiality}

The information gathered in this survey will remain completely confidential and will only be reported in combination with other respondents. Your information will be coded and anonymous.

\section{Contact Information}

If you have any questions regarding this survey, your participation, or your rights as a participant, you may contact the principle investigator, Angela St. Hillaire at angelas@andrews.edu. You may also contact her research advisor, Dr. Elvin Gabriel at gabriel@andrews.edu or (269) 471-6223.

\section{Consent}

Thank you for choosing to support this research with your participation. You may start the survey at any time by clicking on the Continue button below. Clicking the button below is an acknowledgement that you have read and understood this form and are giving your consent to participate in the research described above. 
APPENDIX C

DEMOGRAPHIC DATA SHEET 


\section{DEMOGRAPHIC DATA SHEET}

1. Are you female?
a. Yes
b. No

2. In what year were you born?

3. Which of the following ethnic groups do you most identify with?
a. African American
b. Black Other (please specify:
c. Latino/a American
d. Latino/a other (please specify:
e. Caucasian American
f. Caucasian Other (please specify:
g. Asian American
h. Asian Other (please specify:
i. Bi/Multiracial (please specify:
j. Other (please specify:

4. What is the highest degree or level of school you have completed?
a. High school diploma or less
b. Associate degree
c. Bachelor's degree
d. Master's degree
e. Doctorate degree

5. What was your household income last year?
a. Less than $\$ 20,000$
b. $\$ 20,000$ to $\$ 34,999$
c. $\$ 35,000$ to $\$ 49,999$
d. $\$ 50,000$ to $\$ 74,999$
e. $\$ 75,000$ to $\$ 99,999$
f. $\$ 100,000$ to $\$ 149,999$
g. $\$ 150,000$ or above

6. What is your marital/relationship status?
a. Single
b. Married
c. Domestic partnership
d. Widowed
e. Separated
f. Divorced 
7. With what religious or spiritual group do you identify?
a. Catholic
b. Non-denominational Evangelical
c. Baptist
d. Lutheran
e. Methodist
f. Pentecostal/Charismatic
g. Seventh-Day Adventist
h. Mormon
i. Jehovah's Witness
j. Other (please specify:

8. On the following continuum, please indicate how spiritual and/or religious you are.

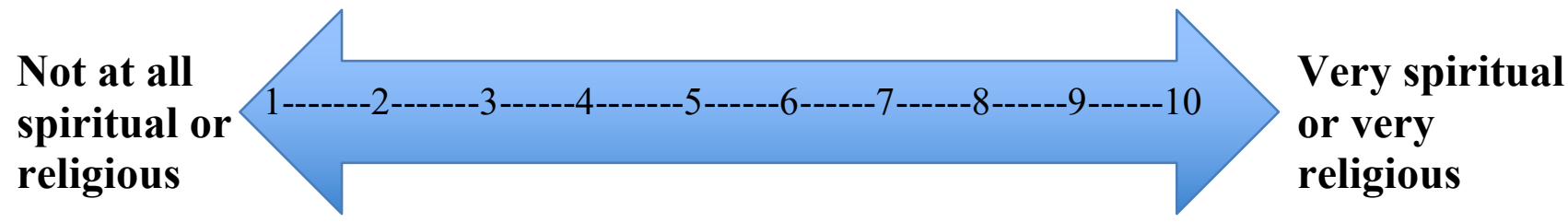


APPENDIX D

CONTEXT OF ABUSE QUESTIONNAIRE 


\section{Context of Abuse Questionnaire}

1. Have you ever been sexually abused by a religious leader?
a. Yes
b. No

2. At what age(s) did the abuse occur? Select all that apply.
a. Childhood (ages 4-10)
b. Adolescence (ages 11-18)
c. Young Adulthood (19-26)
d. Adulthood (27-59)
e. Older adulthood (60 and above)

3. How many times did the abuse occur?
a. One time
b. 2 to 5 times
c. 6 or more times
d. Too many times to remember/can't remember

4. Did the abuse continue over a period of time?
a. Yes
b. No

5. If 'yes' to the previous question, how long did the abuse continue?
a. Under 6 months
b. 6 months to 1 year
c. 1 to 2 years
d. 2 to 5 years
e. More than 5 years

6. Which of the following describes the nature of the abuse? Select all that apply.
a. Fondling or any touching used to evoke sexual feelings, clothed or unclothed
b. Sexually explicit, suggestive, or humiliating comments or language
c. Indecent exposure
d. Exposure to masturbation or other sexual acts
e. Coercion or encouragement to masturbate
f. Penetrative sexual acts
g. Pornography viewing
h. Creating pornographic images or video
i. Forced prostitution
j. Other (please specify:

7. Did the abuse occur by more than one religious leader? 

a. Yes
b. No

8. What was the title/position of the abuser? (If more than one abuser, select all that apply.)
a. Pastor
b. Priest
c. Bishop
d. Elder
e. Deacon
f. Other (please specify:

9. In the two years following the abuse, did you seek or receive counseling?
a. Yes
b. No

10. If yes, how long did the counseling last?
a. Under 6 months
b. 6 months to 1 year
c. 1 to 2 years
d. More than 2 years 
APPENDIX E SURVEYS 


\section{Brief RCOPE}

The following items deal with ways you coped with the negative event in your life. There are many ways to try to deal with problems. These items ask what you did to cope with this negative event. Obviously different people deal with things in different ways, but we are interested in how you tried to deal with it. Each item says something about a particular way of coping. We want to know to what extent you did what the item says. How much or how frequently. Don't answer on the basis of what worked or not-just whether or not you did it. Use these response choices. Try to rate each item separately in your mind from the others. Make your answers as true FOR YOU as you can. Circle the answer that best applies to you.

$1-$ not at all

2 - somewhat

3 - quite a bit

$4-$ a great deal

$(+)$ 1. Looked for a stronger connection with God.

(+) 2. Sought God's love and care.

$(+)$ 3. Sought help from God in letting go of my anger.

$(+)$ 4. Tried to put my plans into action together with God.

(+) 5. Tried to see how God might be trying to strengthen me in this situation.

(+) 6. Asked forgiveness for my sins.

$(+)$ 7. Focused on religion to stop worrying about my problems.

(-) 8. Wondered whether God had abandoned me.

(-) 9. Felt punished by God for my lack of devotion.

(-) 10. Wondered what I did for God to punish me.

(-) 11. Questioned God's love for me.

(-) 12. Wondered whether my church had abandoned me.

(-) 13. Decided the devil made this happen.

(-) 14. Questioned the power of God.

$\begin{array}{llll}1 & 2 & 3 & 4 \\ 1 & 2 & 3 & 4 \\ 1 & 2 & 3 & 4 \\ 1 & 2 & 3 & 4 \\ & & & \\ 1 & 2 & 3 & 4 \\ 1 & 2 & 3 & 4 \\ & & & \\ 1 & 2 & 3 & 4 \\ 1 & 2 & 3 & 4 \\ 1 & 2 & 3 & 4 \\ 1 & 2 & 3 & 4 \\ 1 & 2 & 3 & 4 \\ 1 & 2 & 3 & 4 \\ 1 & 2 & 3 & 4 \\ 1 & 2 & 3 & 4\end{array}$

(+) Positive religious coping item

(-) Negative religious coping item 


\section{Emotion Thermometers (Past)}

In the first four columns, please mark the number (0-10) that best describes how much emotional upset you felt shortly after your experience. In the last column, please indicate how much you needed help for these concerns at that time.

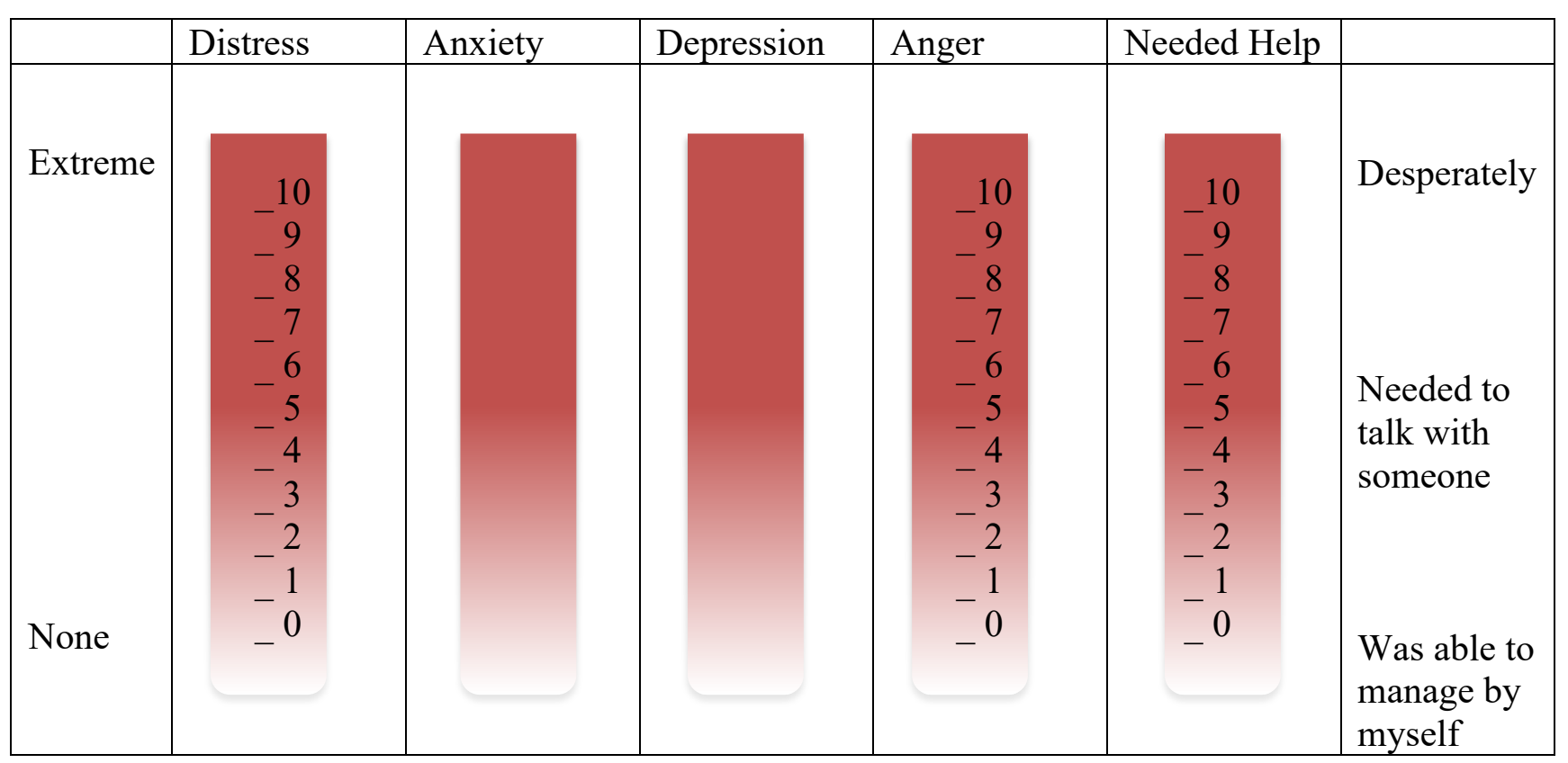




\section{Emotion Thermometers (Present)}

In the first four columns, please mark the number (0-10) that best describes how much emotional upset you have been experiencing in the past month, including today. In the last column, please indicate how much you need help for these concerns.

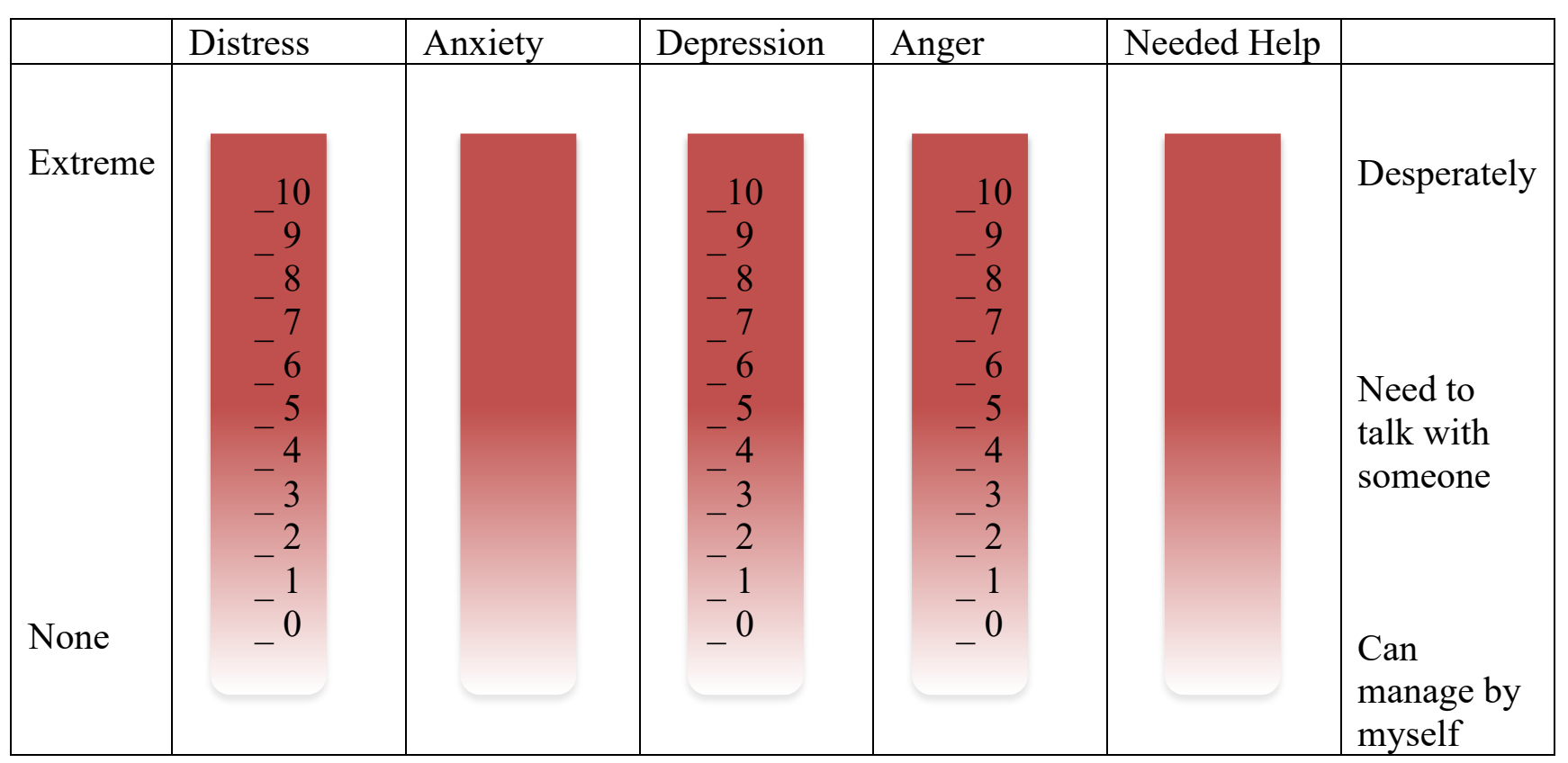




\section{Post Traumatic Growth Inventory}

Client Name: Today's Date:

Indicate for each of the statements below the degree to which this change occurred in your life as a result of the crisis/disaster, using the following scale.

$$
\begin{aligned}
& 0=1 \text { did not experience this change as a result of my crisis. } \\
& 1=1 \text { experienced this change to a very small degree as a result of my crisis. } \\
& 2=1 \text { experienced this change to a small degree as a result of my crisis. } \\
& 3=1 \text { experienced this change to a moderate degree as a result of my crisis. } \\
& 4=1 \text { experienced this change to a great degree as a result of my crisis. } \\
& 5=1 \text { experienced this change to a very great degree as a result of my crisis. }
\end{aligned}
$$

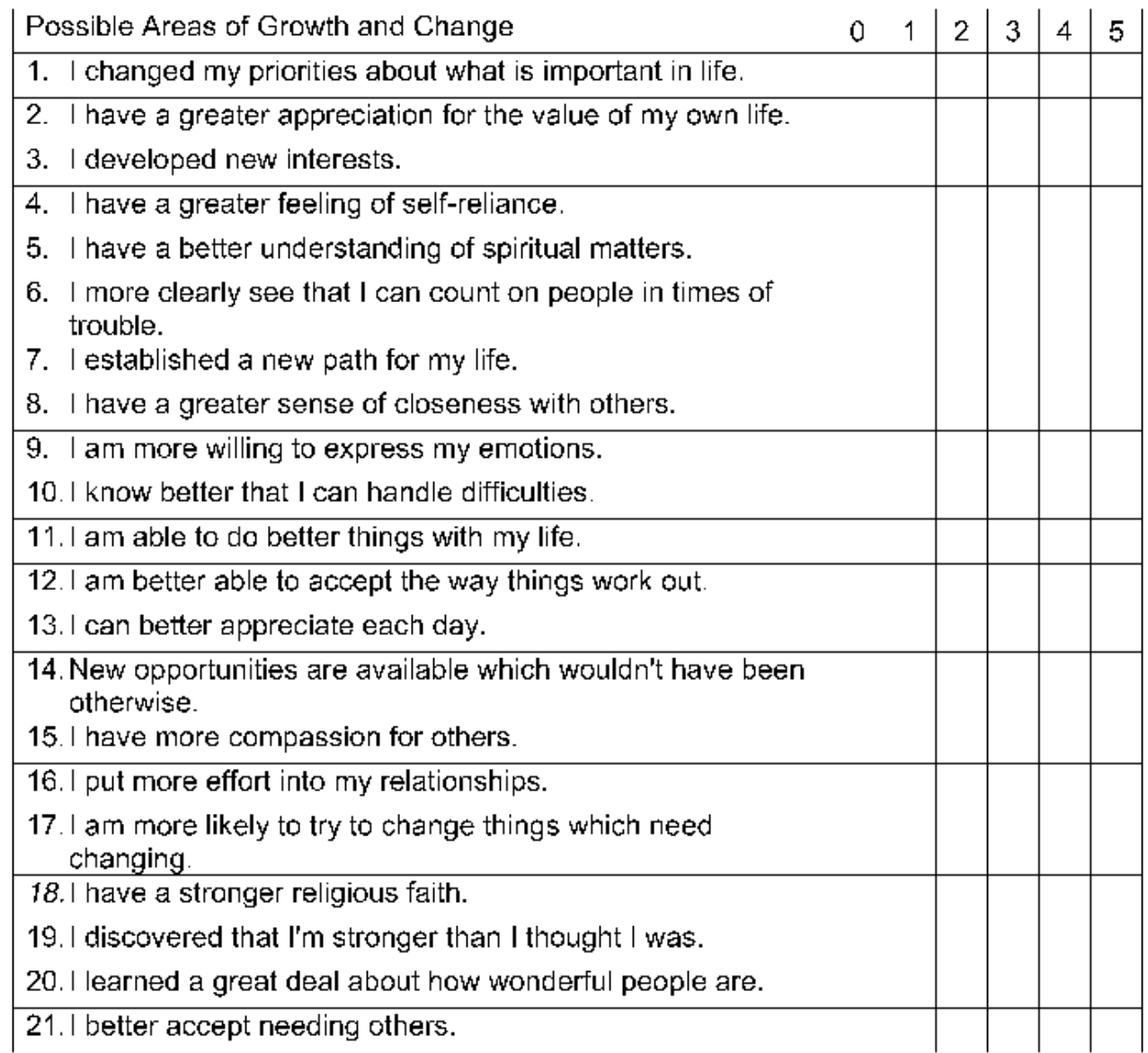




\section{References}

Ahrens, C. E., Abeling, S., Ahmad, S., \& Hinman, J. (2010). Spirituality and well-being: The relationship between religious coping and recovery from sexual assault. Journal of Interpersonal Violence, 25(7), 1242-1263. htpps://doi.org/10.1177/0886260509340533

Ai, A., Hall, D., Pargament, K., \& Tice, T. (2013). Posttraumatic growth in patients who survived cardiac surgery: The predictive and mediating roles of faith-based factors. Journal of Behavioral Medicine, 36(2), 186-198. htpps://doi.org/10.1007/s10865-012-9412-6

Aldwin, C., Levenson, M., \& Spiro, A. (1994). Vulnerability and resilience to combat exposure: Can stress have lifelong effects?. Psychology and Aging, 9, 34-44. htpps://doi.org/10.1037/0882-7974.9.1.34

Angelou, M. (1969,1970). I know why the caged bird sings. Ballantine Books.

Ano, G. G., \& Vasconcelles, E. B. (2005). Religious coping and psychological adjustment to stress: A meta-analysis. Journal of Clinical Psychology, 61(4), 461-480. htpps://doi.org/dx.doi.org/10.1002/jclp.20049

Antonovsky, A. (1993). The structure and properties of the sense of coherence scale. Social Science \& Medicine, 36(6), 725-733. doi:http://dx.doi.org/10.1016/02779536(93)90033-Z

Askay, S. W., \& Magyar-Russell, G. (2009). Post-traumatic growth and spirituality in burn recovery. International Review of Psychiatry, 21(6), 570-579. htpps://doi.org.ezproxy.andrews.edu/10.3109/09540260903344107 
Asukai, N., Kato, H., Kawamura, N., Kim, Y., Yamamato, K., Kishimoto, J., Miyake, Y., \& Nishizono-Maher, A. (2002). Reliability and validity of the Japanese-language version of the impact of event scale-revised (IES-R-J): Four studies of different traumatic events. Journal of Nervous and Mental Disease, 190(3), 175-182. htpps://doi.org/ezproxy.andrews.edu/10.1097/00005053-200203000-00006

Bade, M. K. (2000). Personal growth in the midst of negative life experiences: The role of religious coping strategies and appraisals (Order No. 9980634). Available from ProQuest Dissertations \& Theses Global. (304628374). Retrieved from http://search.proquest.com/docview/304628374?accountid=8313

Baker, J. M., Kelly, C., Calhoun, L. G., Cann, A., \& Tedeschi, R. G. (2008). An examination of posttraumatic growth and posttraumatic depreciation: Two exploratory studies. Journal of Loss \& Trauma, 13(5), 450-465. htpps://doi.org/10.1080/15325020802171367

Bal, S., Van Oost, P., De Bourdeaudhuij, I., \& Crombez, G. (2003). Avoidant coping as a mediator between self-reported sexual abuse and stress-related symptoms in adolescents. Child Abuse \& Neglect, 27(8), 883-897. htpps://doi.org/10.1016/S0145-2134(03)00137-6

Baty, S. D. (2012). Healing the invisible wound: Examining spirituality in the posttraumatic growth of sexual trauma survivors (Order No. 3516060). Available from ProQuest Dissertations \& Theses Global: Health \& Medicine; ProQuest Dissertations \& Theses Global: Social Sciences. (1033007909). http://search.proquest.com/docview/1033007909?accountid=8313 
Bellizzi, K. M. (2003). Understanding the dynamics of posttraumatic growth in breast cancer survivors (Order No. AAI3089739). Available from PsycINFO. (620253189; 2003-95021-017). https://search.proquest.com/docview/620253189?accountid=8313

Benkert, M., \& Doyle, T. (2009). Clericalism, religious duress and its psychological impact on victims of clergy sexual abuse. Pastoral Psychology, 58(3), 223-238. htpps://doi.org/10.1007/s11089-008-0188-0

Bergin, A. E., Masters, K. S., \& Richards, P. S. (1987). Religiousness and mental health reconsidered: A study of an intrinsically religious sample. Journal of Counseling Psychology, 34(2), 197-204. doi:http://dx.doi.org/10.1037/0022-0167.34.2.197

Bilsky, K. D. P. (2013). When the sacred hurts: Using grounded theory analysis to understand how former members experienced abuse of religious authority in Christian groups (Order No. 3576284). Available from ProQuest Dissertations \& Theses Global. (1465384253). Retrieved from http://search.proquest.com/docview/1465384253?accountid=8313

Bitton, M. S. (2014). PTSD, Posttraumatic growth, and coping among ultra-orthodox Jewish battered women in Israel. Journal of Loss \& Trauma, 19(2), 155-172. htpps://doi.org/10.1080/15325024.2012.760383

Bonelli, R., \& Koenig, H. (2013). Mental disorders, religion and spirituality 1990 to 2010: A systematic evidence-based review. Journal of Religion \& Health, 52(2), 657-673. https://doi-org.ezproxy.andrews.edu/10.1007/s10943-013-9691-4 
Boorstein, M., \& Gately, G. (2018, August 14). More than 300 accused priests in Pennsylvania report on Catholic Church sex abuse. The Washington Post. https://www.washingtonpost.com/news/acts-offaith/wp/2018/08/14/pennsylvania-grand-jury-report-on-sex-abuse-in-catholicchurch-will-list-hundreds-of-accused-predator-priests/?utm_term=.733406a16de4

Borja, S. E., Callahan, J. L., \& Long, P. J. (2006). Positive and negative adjustment and social support of sexual assault survivors. Journal of Traumatic Stress, 19(6), 905-914. doi:http://dx.doi.org/10.1002/jts.20169

Brand, B. L., \& Alexander, P. C. (2003). Coping with incest: The relationship between recollections of childhood coping and adult functioning in female survivors of incest. Journal of Traumatic Stress, 16(3), 285.

Bratkovich, K. L. (2010). The relationship of attachment and spirituality with posttraumatic growth following a death loss for college students (Order No. 3422262). Available from ProQuest Dissertations \& Theses Global: Health \& Medicine; ProQuest Dissertations \& Theses Global: Social Sciences. (759096704). Retrieved from http://search.proquest.com/docview/759096704?accountid=8313

Bronfenbrenner, U. (1992). Ecological systems theory. In R. Vasta (Ed.), Six theories of child development: Revised formulations and current issues (pp. 187-249). Jessica Kingsley Publishers.

Bryant, E. (2010). Clergy sex abuse scandal undermines Pope Benedict's crusade to reclaim Europe. Christian Century, 127(9), 15. 
Bryant-Davis, T., Ullman, S. E., Tsong, Y., \& Gobin, R. (2011). Surviving the storm: The role of social support and religious coping in sexual assault recovery of African American women. Violence Against Women, 17(12), 1601-1618. htpps://doi.org/10.1177/1077801211436138

Burke, L. A., Neimeyer, R. A., McDevitt-Murphy, M. E., Ippolito, M. R., \& Roberts, J. M. (2011). Faith in the wake of homicide: Religious coping and bereavement distress in an African American sample. International Journal for The Psychology of Religion, 21(4), 289-307. htpps://doi.org/10.1080/10508619.2011.607416

Buzdar, M., Ali, A., Nadeem, M., \& Nadeem, M. (2015). Relationship between religiosity and psychological symptoms in female university students. Journal of Religion \& Health, 54(6), 2155-2163. htpps://doi.org/10.1007/s10943-014-99410

Cabral, C. M. (2010). Psychological functioning following violence: An examination of posttraumatic growth, distress, and hope among interpersonal violence survivors (Order No. MR72853). Available from ProQuest Dissertations \& Theses Global. (869312610). http://search.proquest.com/docview/869312610?accountid=8313

Cadell, S., Regehr, C., \& Hemsworth, D. (2003). Factors contributing to posttraumatic growth: A proposed structural equation model. American Journal of Orthopsychiatry, 73(3), 279-287. doi:http://dx.doi.org/10.1037/00029432.73 .3 .279

Calhoun, L. G., \& Tedeschi, R. G. (1998). Beyond recovery From Trauma: Implications for clinical practice and research. Journal of Social Issues, 54(2), 357-35731. 
Calhoun, L. G., \& Tedeschi, R. G. (1999). Facilitating posttraumatic growth. Lawrence Erlbaum.

Calhoun, L. G., \& Tedeschi, R. G. (2004). The Foundations of Posttraumatic Growth: New Considerations. Psychological Inquiry, 15(1), 93-102.

Calhoun, L. G., \& Tedeschi, R. G. (2006). The foundations of posttraumatic growth: An expanded framework. In L. G. Calhoun \& R. G. Tedeschi (Eds.), Handbook of posttraumatic growth (pp. 3-23). Lawrence Erlbaum Associates.

Calhoun, L. G., Cann, A., Tedeschi, R. G., \& McMillan, J. (2000). A correlational test of the relationship between posttraumatic growth, religion, and cognitive processing. Journal of Traumatic Stress, 13(3), 521-527.

http://search.proquest.com/docview/619472849?accountid=8313

Campbell, R., Dworkin, E., \& Cabral, G. (2009). An ecological model of the impact of sexual assault on women's mental health. Trauma, Violence, \& Abuse, 10(3), 225-246. doi:http://dx.doi.org/10.1177/1524838009334456

Cann, A., Calhoun, L. G., Tedeschi, R. G., \& Solomon, D. T. (2010). Posttraumatic growth and depreciation as independent experiences and predictors of well-being. Journal of Loss \& Trauma, 15(3), 151-166. doi:10.1080/15325020903375826

Cann, A., Calhoun, L. G., Tedeschi, R. G., Triplett, K. N., Vishnevsky, T., \& Lindstrom, C. M. (2011). Assessing posttraumatic cognitive processes: The Event Related Rumination Inventory. Anxiety, Stress \& Coping, 24(2), 137-156. htpps://doi.org/10.1080/10615806.2010.529901 
Cantón-Cortés, D., Cantón, J., \& Cortés, M. R. (2016). Emotional security in the family system and psychological distress in female survivors of child sexual abuse. Child Abuse \& Neglect, 51, 54-63. https://doiorg.ezproxy.andrews.edu/10.1016/j.chiabu.2015.11.005

Carver, C. S. (1997). You want to measure coping but your protocol's too long: Consider the Brief COPE. International Journal of Behavioral Medicine, 4(1), 92.

Carver, C. S., Scheier, M. F., \& Segerstrom, S. C. (2010). Optimism. Clinical Psychology Review, 30(7), 879-889. htpps://doi.org/10.1016/j.cpr.2010.01.006

Chan, C. S., \& Rhodes, J. E. (2013). Religious coping, posttraumatic stress, psychological distress, and posttraumatic growth among female survivors four years after Hurricane Katrina. Journal of Traumatic Stress, 26(2), 257-265. htpps://doi.org/10.1002/jts.21801

Chen, Y. Y., \& Koenig, H. G. (2006). Traumatic stress and religion: Is there a relationship? A review of empirical findings. Journal of Religion \& Health, 45(3), 371-381. https://doi-org.ezproxy.andrews.edu/10.1007/s10943-006-9040-y

Chopko, B. A. (2010). Posttraumatic distress and growth: An empirical study of police officers. American Journal of Psychotherapy, 64(1), 55-72.

Ciali, P. C. (2006). A two-part investigation: Examining the relationship between spirituality and posttraumatic growth, and the multidimensionality of posttraumatic growth (Order No. 3245814). Available from ProQuest Dissertations \& Theses Global. (304948675). https://search-proquestcom.ezproxy.andrews.edu/docview/304948675?accountid=8313 
Cobb, A. R., Tedeschi, R. G., Calhoun, L. G., \& Cann, A. (2006). Correlates of posttraumatic growth in survivors of intimate partner violence. Journal of Traumatic Stress, 19(6), 895-903. htpps://doi.org/10.1002/jts.20171

Cole, B. S. (2005). Spiritually-focused psychotherapy for people diagnosed with cancer: A pilot outcome study. Mental Health, Religion \& Culture, 8(3), 217-226. htpps://doi.org/10.1080/13694670500138916

Collins, C. M., O’Neill-Arana, M. R., Fontes, L. A., \& Ossege, J. M. (2014). Catholicism and childhood sexual abuse: Women's coping and psychotherapy. Journal of Child Sexual Abuse, 23(5), 519-537. htpps://doi.org/10.1080/10538712.2014.918071

Cordova, M. J., Cunningham, L. L. C., Carlson, C. R., \& Andrykowski, M. A. (2001). Posttraumatic growth following breast cancer: A controlled comparison study. Health Psychology, 20(3), 176-185. doi:http://dx.doi.org.ezproxy.andrews.edu/10.1037/0278-6133.20.3.176

Cotton, S., Puchalski, C. M., Sherman, S. N., Mrus, J. M., Peterman, A. H., Feinberg, J., Pargament, K. I., Justice, A. C., Leonard, A. C., Tsevat, J. (2006). Spirituality and religion in patients with HIV/AIDS. JGIM: Journal of General Internal Medicine, 21, S5-S13. https://doi-org.ezproxy.andrews.edu/10.1111/j.15251497.2006.00642.x

Cudmore, M. J. (2002). The experience of victim suffering and perception of leadership abuse in christian organizations (Order No. MQ77420). Available from ProQuest Dissertations \& Theses Global. (305483270). https://search-proquestcom.ezproxy.andrews.edu/docview/305483270?accountid $=8313$ 
Daly, C. C. (2005). Southern Medical Journal, 98(12), 1238-1239.

Danhauer, S., Russell, G., Tedeschi, R., Jesse, M., Vishnevsky, T., Daley, K., Carroll, S., Triplett, K., Calhoun, L., Cann, A., \& Powell, B. (2013). A longitudinal investigation of posttraumatic growth in adult patients undergoing treatment for acute leukemia. Journal of Clinical Psychology In Medical Settings, 20(1), 13-24. htpps://doi.org/10.1007/s10880-012-9304-5

Davies, A. (2018). From "disorder" to "challenge": Using lifespan development theories to reframe distress. Counselling Psychology Review, 33(1), 24-32. http://search.ebscohost.com.ezproxy.andrews.edu/login.aspx?direct=true $\& d b=a 9 h$ $\& \mathrm{AN}=129773927 \&$ site $=$ ehost-live

Dehan, N., \& Levi, Z. (2009). Spiritual abuse: An additional dimension of abuse experienced by abused Haredi (ultraorthodox) Jewish wives. Violence Against Women, 15(11), 1294-1310. doi:10.1177/1077801209347619

Disch, E., \& Avery, N. (2001). Sex in the consulting room, the examining room, and the sacristy: Survivors of sexual abuse by professionals. American Journal of Orthopsychiatry, 71(2), 204-217. http://dx.doi.org/10.1037/0002-9432.71.2.204

Doehring, C. (1993). Internal desecration: Traumatization and representations of God. University Press of America.

Doyle, T. P. (2006). Clericalism: Enabler of clergy sexual abuse. Pastoral Psychology, 54(3), 189-213. doi:10.1007/s11089-006-6323-x

Doyle, T. P. (2009). The spiritual trauma experienced by victims of sexual abuse by Catholic clergy. Pastoral Psychology, 58, 239-260. htpps://doi.org/10.1007/s11089-008-0187-1 
Drapeau, A., Marchand, A., \& Beaulieu-Prévost, D. (2012). Epidemiology of psychological distress, In Mental Illnesses-Understanding, Prediction and Control, Prof. Luciano LAbate (Ed.), ISBN: 978-953-307-662- 1, InTech, http://www.intechopen.com/books/mental-illnesses-understanding-predictionand- control/epidemiology-of-psychological-distress

Dursun, P., Steger, M. F., Bentele, C., \& Schulenberg, S. E. (2016). Meaning and posttraumatic growth among survivors of the September 2013 Colorado floods. Journal of Clinical Psychology, 72(12), 1247-1263. htpps://doi.org/10.1002/jclp.22344

Easton, S. D., Leone-Sheehan, D. M., \& O’Leary, P. J. (2019). "I will never know the person who I could have become": Perceived changes in self-identity among adult survivors of clergy-perpetrated sexual abuse. Journal of Interpersonal Violence, 34(6), 1139-1162. https://doiorg.ezproxy.andrews.edu/10.1177/0886260516650966

Fallot, R. D., \& Heckman, J. P. (2005). Religious/spiritual coping among women trauma survivors with mental health and substance use disorders. Journal of Behavioral Health Services \& Research, 32(2), 215-226.

Falsetti, S. A., Resick, P. A., \& Davis, J. L. (2003). Changes in religious beliefs following trauma. Journal of Traumatic Stress, 16(4), 391. http://search.ebscohost.com.ezproxy.andrews.edu/login.aspx?direct=true \&db=a9h $\& \mathrm{AN}=10574188 \&$ site $=$ ehost-live 
Farrell, D. P. (2009). Sexual abuse perpetrated by Roman Catholic priests and religious. Mental Health, Religion \& Culture, 12(1), 39-53. htpps://doi.org/10.1080/13674670802116101

Fater, K., \& Mullaney, J. A. (2000). The lived experience of adult male survivors who allege childhood sexual abuse by clergy. Issues in Mental Health Nursing, 21(3), 281-295. htpps://doi.org/10.1080/016128400248095

Feit, A. (2013). Modesty from cats, sexual morality from doves: Orthodox Jewry's silence in the face of sexual misconduct. International Journal of Psychoanalytic Self Psychology, 8(2), 154-172. htpps://doi.org/10.1080/15551024.2013.766950

Fetty, D. G. (2016). Is there justice for sexual trauma? A structural model to examine factors influencing posttraumatic growth and distress (Order No. 10138120). Available from ProQuest Dissertations \& Theses Global. (1805287132). http://search.proquest.com/docview/1805287132?accountid=8313

Flynn, K. A. (2008). In their own voices: Women who were sexually abused by members of the clergy. Journal of Child Sexual Abuse, 17(3-4), 216-237. htpps://doi.org/10.1080/10538710802329684

Fogler, J. M., Shipherd, J. C., Clarke, S., Jensen, J., \& Rowe, E. (2008). The impact of clergy-perpetrated sexual abuse: The role of gender, development, and posttraumatic stress. Journal of Child Sexual Abuse, 17(3/4), 329-358.

Fogler, J. M., Shipherd, J. C., Rowe, E., Jensen, J., \& Clarke, S. (2008). A theoretical foundation for understanding clergy-perpetrated sexual abuse. Journal of Child Sexual Abuse, 17(3/4), 301-328. 
Folkman, S., \& Moskowitz, J. T. (2004). COPING: Pitfalls and promise. Annual Review Of Psychology, 55(1), 745-774. htpps://doi.org/10.1146/annurev.psych.55.090902.141456

Francis, P. C., \& Turner, N. R. (1995). Sexual misconduct within the Christian church: Who are the perpetrators and those they victimize? Counseling \& Values, 39(3), 218.

Frankl, Viktor E. (1959/2006). Man's search for meaning. Boston: Beacon Press.

Franz, T. (2002). Power, patriarchy and sexual abuse in churches of Christian denomination. Traumatology: An International Journal, 8(1), 4-17. doi:http://dx.doi.org/10.1177/153476560200800102

Frazier, P., Tashiro, T., Berman, M., Steger, M., \& Long, J. (2004). Correlates of levels and patterns of positive life changes following sexual assault. Journal of Consulting and Clinical Psychology, 72(1), 19-30. doi:http://dx.doi.org/10.1037/0022-006X.72.1.19

Futa, K. T., Nash, C. L., Hansen, D. J., \& Garbin, C. P. (2003). Adult survivors of childhood abuse: An analysis of coping mechanisms used for stressful childhood memories and current stressors. Journal of Family Violence, 18(4), 227-239.

Gall, T. L. (2000). Integrating religious resources within a general model of stress and coping: Long-term adjustment to breast cancer. Journal of Religion \& Health, 39(2), 167.

Gall, T. L. (2006). Spirituality and coping with life stress among adult survivors of childhood sexual abuse. Child Abuse \& Neglect, 30(7), 829-844. htpps://doi.org/10.1016/j.chiabu.2006.01.003 
Gall, T. L., Basque, V., Damasceno-Scott, M., \& Vardy, G. (2007). Spirituality and the current adjustment of adult survivors of childhood sexual abuse. Journal for the Scientific Study of Religion, 46(1), 101-117. htpps://doi.org/10.1111/j.14685906.2007.00343.x

Gall, T. L., Charbonneau, C., Clarke, N. H., Grant, K., Joseph, A., \& Shouldice, L. (2005). Understanding the nature and role of spirituality in relation to coping and health: A conceptual framework. Canadian Psychology/Psychologie Canadienne, 46(2), 88-104. doi:http://dx.doi.org/10.1037/h0087008

Gall, T. L., Malette, J., \& Guirguis-Younger, M. (2011). Spirituality and religiousness: A diversity of definitions. Journal of Spirituality In Mental Health, 13(3), 158-181. htpps://doi.org/10.1080/19349637.2011.593404

Ganje-Fling, M., Veach, P. M., Kuang, H., \& Houg, B. (2000). Effects of childhood sexual abuse on client spiritual well-being. Counseling \& Values, 44(2), 84.

Ganzevoort, R. R. (2001). Religion in rewriting the story: Case study of a sexually abused man. International Journal for the Psychology Of Religion, 11(1), 45-62.

García, F. E., Duque, A., \& Cova, F. (2017). The four faces of rumination to stressful events: A psychometric analysis. Psychological Trauma: Theory, Research, Practice, and Policy, 9(6), 758-765. doi:http://dx.doi.org.ezproxy.andrews.edu/10.1037/tra0000289

Gardner, M. H., Mrug, S., Schwebel, D. C., Phipps, S., Whelan, K., \& Madan-Swain, A. (2017). Demographic, medical, and psychosocial predictors of benefit finding among caregivers of childhood cancer survivors. Psycho-Oncology, 26(1), 125132. htpps://doi.org/10.1002/pon.4014 
George, L. K., Larsons, D. B., Koeing, H. G., \& McCullough, M. E. (2000). Spirituality and health: What we know, what we need to know. Journal of Social and Clinical Psychology, 19(1), 102-116. https://search.proquest.com/docview/224876860?accountid=8313

Ghafouri, S. F., Ghanbari, S., Fallahzadeh, H., \& Shokri, O. (2016). The relation between marital adjustment and posttraumatic growth in infertile couples: The mediatory role of religious coping strategies. Journal of Reproduction \& Infertility, 17(4), $221-229$.

Glaser, R. D., \& Thorpe, J. S. (1986). Unethical intimacy: A survey of sexual contact and advances between psychology educators and female graduate students. American Psychologist, 41(1), 43-51. doi:http://dx.doi.org/10.1037/0003-066X.41.1.43

Goldenberg, R. (2013). Unholy clergy: Amending state child abuse reporting statutes to include clergy members as mandatory reporters in child sexual abuse cases. Family Court Review, 51(2), 298-315. htpps://doi.org/10.1111/fcre.12028

Grad, R. I., \& Zeligman, M. (2017). Predictors of post-traumatic growth: The role of social interest and meaning in life. Journal of Individual Psychology, 73(3), 190207.

Graves, S. L. (2015). Clergy-perpetrated child sexual abuse: Perceived effects based on archival reports of adult survivors who pursued litigation against the catholic church (Order No. 3711590). Available from ProQuest Dissertations \& Theses Global. (1707928886). http://search.proquest.com/docview/1707928886?accountid=8313 
Greenberg, M. A. (1995). Cognitive processing of traumas: The role of intrusive thoughts and reappraisals. Journal of Applied Social Psychology, 25(14), 1262-1295.

Grubaugh, A. L., \& Resick, P. A. (2007). Posttraumatic growth in treatment-seeking female assault victims. Psychiatric Quarterly, 78(2), 145-155. htpps://doi.org/10.1007/s11126-006-9034-7

Gubi, P. M., \& Jacobs, R. (2009). Exploring the impact on counsellors of working with spiritually abused clients. Mental Health, Religion \& Culture, 12(2), 191-204. htpps://doi.org/10.1080/13674670802441509

Guido, J. J. (2008). A unique betrayal: Clergy sexual abuse in the context of the Catholic religious tradition. Journal of Child Sexual Abuse, 17(3/4), 255-269.

Harris, R. C. (1995). The role of religion in heart-transplant recipients' long-term health and well-being. Journal of Religion and Health, 34(1), 17-32.

Hartley, S., Johnco, C., Hofmeyr, M., \& Berry, A. (2016). The nature of posttraumatic growth in adult survivors of child sexual abuse. Journal of Child Sexual Abuse, 25(2), 201-220. htpps://doi.org/10.1080/10538712.2015.1119773

Hassija, C. M., \& Turchik, J. A. (2016). An examination of disclosure, mental health treatment use, and posttraumatic growth among college women who experienced sexual victimization. Journal of Loss \& Trauma, 21(2), 124-136. https://doiorg.ezproxy.andrews.edu/10.1080/15325024.2015.1011976

Helgeson, V. S., Reynolds, K. A., \& Tomich, P. L. (2006). A meta-analytic review of benefit finding and growth. Journal of Consulting \& Clinical Psychology, 74(5), 797-816. 
Henderson, A., \& Ellison, C. (2015). My body is a temple: Eating disturbances, religious involvement, and mental health among young adult women. Journal of Religion \& Health, 54(3), 954-976. htpps://doi.org/10.1007/s10943-014-9838-y

Hill, P. C., Pargament, K. I., Hood, J. W., McCullough, M. E., Swyers, J. P., Larson, D. B., \& Zinnbauer, B. J. (2000). Conceptualizing religion and spirituality: Points of commonality, points of departure. Journal for the Theory of Social Behaviour, 30(1), 51 .

Hillberg, T., Hamilton-Giachritsis, C., \& Dixon, L. (2011). Review of meta-analyses on the association between child sexual abuse and adult mental health difficulties: A systematic approach. Trauma, Violence \& Abuse, 12(1), 38-49. https://doiorg.ezproxy.andrews.edu/10.1177/1524838010386812

Hobfoll, S. E., Hall, B. J., Canetti-Nisim, D., Galea, S., Johnson, R. J., \& Palmieri, P. A. (2007). Refining our understanding of traumatic growth in the face of terrorism: Moving from meaning cognitions to doing what is meaningful. Applied Psychology: An International Review, 56(3), 345-366. htpps://doi.org/10.1111/j.1464-0597.2007.00292.x

Horwitz, A. V. (2007). Distinguishing distress from disorder as psychological outcomes of stressful social arrangements. Health: An Interdisciplinary Journal for the Social Study of Health, Illness \& Medicine, 11(3), 273-289. https://doiorg.ezproxy.andrews.edu/10.1177/1363459307077541

Hu, L., \& Bentller, P. M. (1999). Cutoff criteria for fit indices in covariance structure analysis: Conventional criteria versus... Structural Equation Modeling, 6(1), 1. https://doi-org.ezproxy.andrews.edu/10.1080/10705519909540118 
Hughes, K. L., Sargeant, H., \& Hawkes, A. L. (2011). Acceptability of the Distress Thermometer and Problem List to community-based telephone cancer helpline operators, and to cancer patients and carers. BMC Cancer, 11(1), 46-53. htpps://doi.org/10.1186/1471-2407-11-46

Huson, D. P. (2002). Psychological, sexual, and spiritual effects of clergy sexual abuse of women (Order No. 3071235). Available from ProQuest Dissertations \& Theses Global. (276280649). http://search.proquest.com/docview/276280649?accountid=8313

Idler, E., Blevins, J., Kiser, M., \& Hogue, C. (2017). Religion, a social determinant of mortality? A 10-year follow-up of the Health and Retirement Study. PLoS ONE, 12(12), 1-15. https://doi-org.ezproxy.andrews.edu/10.1371/journal.pone.0189134

Israel-Cohen, Y., Kaplan, O., Noy, S., \& Kashy-Rosenbaum, G. (2016). Religiosity as a moderator of self-efficacy and social support in predicting traumatic stress among combat soldiers. Journal of Religion \& Health, 55(4), 1160-1171. https://doiorg.ezproxy.andrews.edu/10.1007/s10943-016-0187-x

Janoff-Bulman, R. (2004). Posttraumatic growth: Three explanatory models. Psychological Inquiry, 15(1), 30-43.

Karanci, A. N., İşıklı, S., Aker, A. T., Gül, E. İ., Erkan, B. B., Özkol, H., \& Güzel, H. Y. (2012). Personality, posttraumatic stress and trauma type: Factors contributing to posttraumatic growth and its domains in a Turkish community sample. European Journal of Psychotraumatology, 31-14. 
Kennedy, J. E., Davis, R. C., \& Taylor, B. G. (1998). Changes in spirituality and wellbeing among victims of sexual assault. Journal for the Scientific Study of Religion, 37(2), 322. https://doi-org.ezproxy.andrews.edu/10.2307/1387531

Kilpatrick, D. G., Acierno, R., Resnick, H. S., Saunders, B. E., \& Best, C. L. (1997). A 2year longitudinal analysis of the relationships between violent assault and substance use in women. Journal of Consulting and Clinical Psychology, 65(5), 834-847. doi:http://dx.doi.org/10.1037/0022-006X.65.5.834

Kobasa, S. C. (1979). Stressful life events, personality, and health: An inquiry into hardiness. Journal of Personality and Social Psychology, 37(1), 1-11. doi:http://dx.doi.org/10.1037/0022-3514.37.1.1

Kobasa, S. C., Maddi, S. R., Puccetti, M. C., \& Zola, M. A. (1985). Effectiveness of hardiness, exercise and social support as resources against illness. Journal of Psychosomatic Research, 29(5), 525-533. doi:http://dx.doi.org/10.1016/00223999(85)90086-8

Koenig, H. G. (1997). Is religion good for your health? The effects of religion on physical and mental health. Haworth Press.

Koenig, H. G., \& Cohen, H. J. (1992). Religious coping and depression among elderly, hospitalized medically ill men. American Journal of Psychiatry, 149(12), 16931700.

Koenig, H. G., Pargament, K. I., \& Nielsen, J. (1998). Religious coping and health status in medically ill hospitalized older adults. Journal of Nervous and Mental Disease, 186(9), 513-521. doi:http://dx.doi.org.ezproxy.andrews.edu/10.1097/00005053199809000-00001 
Kopacz, M., Morley, S., Woźniak, B., Simons, K., Bishop, T., \& Vance, C. (2016).

Religious well-being and suicide ideation in veterans-An exploratory study. Pastoral Psychology, 65(4), 481-491. htpps://doi.org/10.1007/s11089-016-0699-z

Krause, N., Pargament, K. I., \& Ironson, G. (2017). Does a religious transformation buffer the effects of lifetime trauma on happiness? International Journal for the Psychology of Religion, 27(2), 104-115. htpps://doi.org/1080/10508619.2017.1300506

Krumrei, E. J., Mahoney, A., \& Pargament, K. I. (2009). Divorce and the divine: The role of spirituality in adjustment to divorce. Journal of Marriage \& Family, 71(2), 373-383. htpps://doi.org/10.1111/j.1741-3737.2009.00605.x

Laufer, A., Raz-Hamma, Y., Levine, S. Z., \& Solomon, Z. (2009). Posttraumatic growth in adolescence: The role of religiosity, distress and forgiveness. Journal of Social \& Clinical Psychology, 28(7), 862-880.

Laufer, A., \& Solomon, Z. (2006). Posttraumatic symptoms and posttraumatic growth among Israeli youth exposed to terror incidents. Journal of Social \& Clinical Psychology, 25(4), 429-447.

Lee, E. O. (2007). Religion and spirituality as predictors of well-being among Chinese American and Korean American older adults. Journal of Religion, Spirituality \& Aging, 19(3), 77-100.

Leeman, M. S., Dispenza, F., \& Chang, C. Y. (2015). Lifestyle as a predictor of posttraumatic growth. Journal of Individual Psychology, 71(1), 58-74. 
Levin, J. (2012). Religion and positive well-being among Israeli and diaspora Jews: Findings from the World Values Survey. Mental Health, Religion \& Culture, 15(7), 709-720. htpps://doi.org/10.1080/13674676.2011.617002

Levin, J. (2014). Religion and happiness among Israeli Jews: Findings from the ISSP Religion III Survey. Journal of Happiness Studies, 15(3), 593-611. htpps://doi.org/10.1007/s10902-013-9437-8

Lev-Wiesel, R., Amir, M., \& Besser, A. (2005). Posttraumatic growth among female survivors of childhood sexual abuse in relation to the perpetrator identity. Journal of Loss \& Trauma, 10(1), 7-17. htpps://doi.org/10.1080/I532502049089060G

Linley, P. A., \& Joseph, S. (2004). Positive change following trauma and adversity: A review. Journal of Traumatic Stress, 17(1), 11-21.

Luterek, J. A., Bittinger, J. N., \& Simpson, T. L. (2011). Posttraumatic sequelae associated with military sexual trauma in female veterans enrolled in VA Outpatient Mental Health Clinics. Journal of Trauma \& Dissociation, 12(3), 261274. https://doi-org.ezproxy.andrews.edu/10.1080/15299732.2011.551504

Marcel, M. (2013). Victim gender in news coverage of the priest sex crisis by the Boston Globe. Women's Studies In Communication, 36(3), 288-311.

Marler, P. L., \& Hadaway, C. K. (2002). "Being religious" or "being spiritual” in America: A zero-sum proposition? Journal for the Scientific Study of Religion, 41(2), 289-300.

http://search.ebscohost.com.ezproxy.andrews.edu/login.aspx?direct=true $\& d b=a 9 h$ $\& \mathrm{AN}=6604915 \&$ site $=$ ehost-live 
Marotta-Walters, S. (2015). Spiritual meaning making following clergy-perpetrated sexual abuse. Traumatology: An International Journal, 21(2), 64-70. doi:http://dx.doi.org/10.1037/trm0000022 doi:10.1080/07491409.2013.832088

Matthews, L. T., \& Marwit, S. J. (2003). Examining the assumptive world views of parents bereaved by accident, murder, and illness. Omega: Journal of Death $\&$ Dying, 48(2), 115-136.

http://search.ebscohost.com.ezproxy.andrews.edu/login.aspx?direct=true $\& \mathrm{db}=\mathrm{a} 9 \mathrm{~h}$ $\& \mathrm{AN}=13150083 \&$ site $=$ ehost-live

McConnell, K. M., Pargament, K. I., Ellison, C. G., \& Flannelly, K. J. (2006). Examining the links between spiritual struggles and symptoms of psychopathology in a national sample. Journal of Clinical Psychology, 62(12), 1469-1484. htpps://doi.org/10.1002/jclp.20325

McMackin, R. A., Keane, T. M., \& Kline, P. M. (2008). Introduction to special issue on betrayal and recovery: Understanding the trauma of clergy sexual abuse. Journal of Child Sexual Abuse, 17(3/4), 197-200.

Meyers, L. S., Gamst, G., \& Guarino, A. J. (2013). Applied multivariate research: Design and interpretation (2nd ed.). SAGE. htpps://doi.org/9781412988117

Milam, J. E., Ritt-Olson, A., \& Unger, J. B. (2004). Posttraumatic growth among adolescents. Journal of Adolescent Research, 19(2), 192-204. doi:http://dx.doi.org.ezproxy.andrews.edu/10.1177/0743558403258273

Milam, S. R. B., \& Schmidt, C. K. (2018). A mixed methods investigation of posttraumatic growth in young adults following parental divorce. Family Journal, 26(2), 156-165. https://doi-org.ezproxy.andrews.edu/10.1177/1066480718781518 
Mitchell, A. J. (2007). Pooled results from 38 analyses of the accuracy of distress thermometer and other ultra-short methods of detecting cancer-related mood disorder. Journal of Clinical Oncology, 25, 4670-4681. htpps://doi.org/10.1200/JCO.2006.10.0438

Mitchell, A. J. (2008). The clinical significance of subjective memory complaints in the diagnosis of mild cognitive impairment and dementia: a meta-analysis. International Journal of Geriatric Psychiatry, 23(11), 1191-1202. htpps://doi.org/10.1002/gps.2053

Mitchell, A. J., Morgan, J. P., Petersen, D., Fabbri, S., Fayard, C., Stoletniy, L., \& Chiong, J. (2012). Validation of simple visual-analogue thermometer screen for mood complications of cardiovascular disease: The Emotion Thermometers. Journal of Affective Disorders, 136(3), 1257-1263. https://doiorg.ezproxy.andrews.edu/10.1016/j.jad.2011.06.008

Mosher, C. E., Danoff-Burg, S., \& Brunker, B. (2006). Post-traumatic growth and psychosocial adjustment of daughters of breast cancer survivors. Oncology Nursing Forum, 33(3), 543-551. htpps://doi.org/10.1188/06.ONF.543-551

Neimeyer, R. A. (2004). Fostering posttraumatic growth: A narrative elaboration. Psychological Inquiry, 15(1), 53-59. http://search.ebscohost.com.ezproxy.andrews.edu/login.aspx?direct=true $\& d b=a 9 h$ $\& \mathrm{AN}=12650859 \&$ site $=$ ehost-live

Neimeyer, R., Herrero, O., \& Botella, L. (2006). Chaos to coherence: Psychotherapeutic integration of traumatic Loss. Journal of Constructivist Psychology, 19(2), 127145. htpps://doi.org/10.1080/10720530500508738 
Neustein, A., \& Lesher, M. (2008). A single-case study of rabbinic sexual abuse in the Orthodox Jewish community. Journal of Child Sexual Abuse, 17(3/4), 270-289. https://doi-org.ezproxy.andrews.edu/10.1080/10538710802329783

Nietzsche, F. (1889/2009). Twilight of the idols: Or how to philosophize with a hammer. Oxford University Press.

O’Leary, V. E. \& Ickovics, J. R. (1995). Resilience and thriving in response to challenge: An opportunity for a paradigm shift in women's health. Women's Health, 1(2), 121-142. PMID:9373376

Orchowski, L. M., \& Gidycz, C. A. (2012). To whom do college women confide following sexual assault? A prospective study of predictors of sexual assault disclosure and social reactions. Violence Against Women, 18(3), 264-288. doi:http://dx.doi.org/10.1177/1077801212442917

Orchowski, L. M., Untied, A. S., \& Gidycz, C. A. (2013). Social reactions to disclosure of sexual victimization and adjustment among survivors of sexual assault. Journal of Interpersonal Violence, 28(10), 2005-2023. doi:http://dx.doi.org/10.1177/0886260512471085

Pals, J. L., \& McAdams, D. P. (2004). The transformed self: A narrative understanding of posttraumatic growth. Psychological Inquiry, 15(1), 65-69.

Pargament, K. I. (1997). The psychology of religion and coping: Theory, research, and practice. Guilford Press.

Pargament, K. I. (1999). The psychology of religion and spirituality? Yes and no. International Journal for the Psychology of Religion, 9(1), 3. 
Pargament, K., Feuille, M., \& Burdzy, D. (2011). The Brief RCOPE: Current psychometric status of a short measure of religious coping. Religions, 2(1), 5176. doi:http://dx.doi.org/10.3390/rel2010051

Pargament, K. I., Ishler, K., Dubow, E. F., Stanik, P., Rouiller, R., Crowe, P., Cullman, E., Albert, M., \& Royster, B. J. (1994). Methods of religious coping with the Gulf War: Cross-sectional and longitudinal analyses. Journal for the Scientific Study of Religion, 33(4), 347.

Pargament, K. I., Koenig, H. G., \& Perez, L. (2000). The many methods of religious coping: Development and initial validation of the RCOPE. Journal of Clinical Psychology, 56(4), 519-543.

Pargament, K. I., Koenig, H. G., Tarakeshwar, N., \& Hahn, J. (2004). Religious coping methods as predictors of psychological, physical and spiritual outcomes among medically ill elderly patients: A two-year longitudinal study. Journal of Health Psychology 9(6), 713-730. htpps://doi.org/10.1177/1359105304045366

Pargament, K. I., Magyar, G. M., Benore, E., \& Mahoney, A. (2005). Sacrilege: A study of sacred loss and desecration and their implications for health and well-being in a community sample. Journal for the Scientific Study of Religion, 44(1), 59-78. https://doi-org.ezproxy.andrews.edu/10.1111/j.1468-5906.2005.00265.x

Pargament, K. I., Magyar-Russell, G. M., \& Murray-Swank, N. A. (2005). The sacred and the search for significance: Religion as a unique process. Journal of Social Issues, 61(4), 665-687. htpps://doi.org/10.1111/j.1540-4560.2005.00426.x 
Pargament, K. I., Murray-Swank, N., \& Mahoney, A. (2008). Problem and solution: The spiritual dimension of clergy sexual abuse and its impact on survivors. Journal of Child Sexual Abuse: Research, Treatment, \& Program Innovations for Victims, Survivors, \& Offenders, 17(3-4), 397-420. https://search.proquest.com/docview/621690109?accountid=8313

Pargament, K. I., \& Raiya, H. A. (2007). A decade of research on the psychology of religion and coping: Things we assumed and lessons we learned. Psyke \& Logos, $28(2), 742-766$.

https://search.proquest.com/docview/622063071 accountid=8313

Pargament, K. I., Smith, B. W., Koenig, H. G., \& Perez, L. (1998). Patterns of positive and negative religious coping with major life stressors. Journal for the Scientific Study of Religion, 37(4), 710-724.

Pargament, K. I., Zinnbauer, B. J., Scott, A. B., Butter, E. M., Zerowin, J., \& Stanik, P. (2003). Red flags and religious coping: Identifying some religious warning signs among people in crisis $<$ FNR $>1</$ FNR $><$ FN $>$ Originally published in Volume 54, Number 1, 1998, pages 77-89. </FN>. Journal of Clinical Psychology, 59(12), $1335-1348$.

Park, C. L., \& Cohen, L. H. (1993). Religious and nonreligious coping with the death of a friend. Cognitive Therapy \& Research, 17(6), 561-577.

Park, C. L., Cohen, L. H., \& Murch, R. L. (1996). Assessment and prediction of stressrelated growth. Journal of Personality, 64(1), 71-105. htpps://doi.org/10.1111/1467-6494.ep9606210694 
Park, C. L., Edmondson, D., \& Blank, T. O. (2009). Religious and non-religious pathways to stress-related growth in cancer survivors. Applied Psychology: Health \& Well-Being, 1(3), 321-335. https://doiorg.ezproxy.andrews.edu/10.1111/j.1758-0854.2009.01009.x

Phillips, M. R. (2009). Is distress a symptom of mental disorders, a marker of impairment, both or neither? World Psychiatry, 8(2), 91-92.

Powell, S., Rosner, R., Butolo, W., Tedeschi, R. G., \& Calhoun, L. G. (2003). Posttraumatic growth after war: A study with former refugees and displaced people in Sarajevo. Journal of Clinical Psychology, 59(1), 71-83.

Prati, G., \& Pietrantoni, L. (2009). Optimism, social support, and coping strategies as factors contributing to posttraumatic growth: A meta-analysis. Journal of Loss \& Trauma, 14(5), 364-388. htpps://doi.org/10.1080/15325020902724271

Proffitt, D., Cann, A., Calhoun, L., \& Tedeschi, R. (2007). Judeo-Christian clergy and personal crisis: Religion, posttraumatic growth and well being. Journal of Religion \& Health, 46(2), 219-231. htpps://doi.org/10.1007/s10943-006-9074-1

Psychological distress. (n.d.) McGraw-Hill Concise Dictionary of Modern Medicine. (2002). Retrieved June 8, 2017 from http://medicaldictionary.thefreedictionary.com/psychological+distress

Rahm, G., Renck, B., \& Ringsberg, K. C. (2013). Psychological distress among women who were sexually abused as children. International Journal of Social Welfare, 22(3), 269-278. https://doi-org.ezproxy.andrews.edu/10.1111/j.14682397.2012.00898.x 
Ramirez, S. P., Macêdo, D. S., Sales, P. M. G., Figueiredo, S. M., Daher, E. F., Araújo, S. M., Pargament, K., Hyphantis, T. N., Carvalho, A. F. (2012). The relationship between religious coping, psychological distress and quality of life in hemodialysis patients. Journal of Psychosomatic Research, 72(2), 129-135. doi:http://dx.doi.org/10.1016/j.jpsychores.2011.11.012

Rauch, M. (2009). Healing the soul after religious abuse: The dark heaven of recovery Praeger Publishers/Greenwood Publishing Group. https://search.proquest.com/docview/622064405?accountid=8313

Richards, A. C. (2004). Sexual misconduct by clergy in the Episcopal Church. Studies In Gender \& Sexuality, 5(2), 139-165.

Ridner S. H. (2004). Psychological distress: concept analysis. Journal of Advanced Nursing, 45(5), 536-545. https://doi-org.ezproxy.andrews.edu/10.1046/j.13652648.2003.02938.x

Ronneberg, C. R., Miller, E. A., Dugan, E., \& Porell, F. (2016). The protective effects of religiosity on depression: A 2-year prospective study. Gerontologist, 56(3), 421431. htpps://doi.org/10.1093/geront/gnu073

Ryrie, I., \& Norman, I. (2013). The origins and expression of psychological distress. In I. Noman \& I. Ryrie (Eds.), The art and science of mental health nursing: A textbook of principles and practice, 3rd ed. (pp. 3-34). Open University Press. http://www.mheducation.co.uk/openup/chapters/0335215882.pdf 
Schaefer, J. A., \& Moos, R. H. (1998). The context for posttraumatic growth: Life crises, individual and social resources, and coping. In R. G. Tedeschi, C. L. Park \& L. G. Calhoun (Eds.), Posttraumatic growth: Positive changes in the aftermath of crisis (pp. 99-125, Chapter viii, 258 Pages) Lawrence Erlbaum Associates Publishers. https://search-proquestcom.ezproxy.andrews.edu/docview/619351069?accountid=8313

Schaefer, L. M., Howell, K. H., Schwartz, L. E., Bottomley, J. S., \& Crossnine, C. B. (2018). A concurrent examination of protective factors associated with resilience and posttraumatic growth following childhood victimization. Child Abuse \& Neglect, 85, 17-27. https://doiorg.ezproxy.andrews.edu/10.1016/j.chiabu.2018.08.019

Scheier, M. F., \& Carver, C. S. (1985). Optimism, coping, and health: Assessment and implications of generalized outcome expectancies. Health Psychology, 4(3), 219247. doi:http://dx.doi.org/10.1037/0278-6133.4.3.219

Schnell, T., \& Becker, P. (2006). Personality and meaning in life. Personality and Individual Differences, 41(1), 117-129. doi:http://dx.doi.org/10.1016/j.paid.2005.11.030

Shakespeare-Finch, J., \& Barrington, A. J. (2012). Behavioural changes add validity to the construct of posttraumatic growth. Journal of Traumatic Stress, 25(4), 433439. https://doi-org.ezproxy.andrews.edu/10.1002/jts. 21730

Shakespeare-Finch, J., \& de Dassel, T. (2009). Exploring posttraumatic outcomes as a function of childhood sexual abuse. Journal of Child Sexual Abuse, 18(6), 623640. htpps://doi.org/10.1080/10538710903317224 
Shakespeare-Finch, J., \& Enders, T. (2008). Corroborating evidence of posttraumatic growth. Journal of Traumatic Stress, 21(4), 421-424. https://doiorg.ezproxy.andrews.edu/10.1002/jts. 20347

Shaw, A., Joseph, S., \& Linley, P. A. (2005). Religion, spirituality, and posttraumatic growth: A systematic review. Mental Health, Religion \& Culture, 8(1), 1-11. htpps://doi.org/10.1080/1367467041000157981

Shea, D. J. (2008). Effects of sexual abuse by Catholic Priests on adults victimized as children. Sexual Addiction \& Compulsivity, 15(3), 250-268. htpps://doi.org/10.1080/10720160802288993

Skaczkowski, G., Hayman, T., Strelan, P., Miller, J., \& Knott, V. (2013). Complementary medicine and recovery from cancer: the importance of post-traumatic growth. European Journal of Cancer Care, 22(4), 474-483. htpps://doi.org/10.1111/ecc.12053

Smigelsky, M. A., Gill, A. R., Foshager, D., Aten, J. D., \& Im, H. (2017). "My heart is in his hands": The lived spiritual experiences of Congolese refugee women survivors of sexual violence. Journal of Prevention \& Intervention In the Community, 45(4), 261-273. htpps://doi.org/10.1080/10852352.2016.1197754

Sodhi, R., \& Manju. (2014). Spirituality and religiosity as predictors of mental and physical health. Indian Journal of Health \& Wellbeing, 5(2), 273-278. http://search.ebscohost.com.ezproxy.andrews.edu/login.aspx?direct=true $\& d b=a 9 h$ $\& \mathrm{AN}=97584910 \&$ site $=$ ehost-live 
Southwick, S. M., Bonanno, G. A., Masten, A. S., Panter-Brick, C., \& Yehuda, R. (2014). Resilience definitions, theory, and challenges: interdisciplinary perspectives. European Journal of Psychotraumatology, 51-14. htpps://doi.org/10.3402/ejpt.v5.25338

Steger, M. F., Owens, G. P., \& Park, C. L. (2015). Violations of War: Testing the Meaning-Making Model Among Vietnam Veterans. Journal of Clinical Psychology, 71(1), 105-116. htpps://doi.org/10.1002/jclp.22121

Tabachnick, B. G., \& Fidell, L. S. (2007). Using multivariate statistics (5 $5^{\text {th }}$ ed.). Allyn \& Bacon.

Taku, K., Calhoun, L. G., Cann, A., \& Tedeschi, R. G. (2008). The role of rumination in the coexistence of distress and posttraumatic growth among bereaved Japanese university students. Death Studies, 32(5), 428-444. htpps://doi.org/10.1080/07481180801974745

Taku, K., Cann, A., Calhoun, L. G., \& Tedeschi, R. G. (2008). The factor structure of the posttraumatic growth inventory: A comparison of five models using confirmatory factor analysis. Journal of Traumatic Stress, 21(2), 158-164. https://doiorg.ezproxy.andrews.edu/10.1002/jts.20305

Taku, K., Cann, A., Tedeschi, R. G., \& Calhoun, L. G. (2009). Intrusive versus deliberate rumination in posttraumatic growth across U.S. and Japanese samples. Anxiety, Stress \& Coping, 22(2), 129-136. htpps://doi.org/10.1080/10615800802317841

Tedeschi, R. G. (1999). Violence transformed: Posttraumatic growth in survivors and their societies. Aggression and Violent Behavior, 4(3), 319-341. http://search.proquest.com/docview/619413661 ?accountid=8313 
Tedeschi, R. G., \& Calhoun, L. G. (1996). The Posttraumatic Growth Inventory: Measuring the positive legacy of trauma. Journal of Traumatic Stress, 9(3), 455471.

http://66.199.228.237/boundary/addiction/boundary/PosttraumaticGrowthInventor y.pdf

Tedeschi, R. G., \& Calhoun, L. (2004a). Posttraumatic growth: A new perspective on psychotraumatology. Psychiatric Times, 21(4), 58-60.

Tedeschi, R. G., \& Calhoun, L. G. (2004b). Posttraumatic growth: Conceptual foundations and empirical evidence. Psychological Inquiry, 15(1), 1-18.

Tedeschi, R. G., \& Calhoun, L. G. (2006). Time of change? The spiritual challenges of bereavement and loss. Omega: Journal of Death \& Dying, 53(1/2), 105-116.

Tedeschi, R. G., Calhoun, L. G., \& Cann, A. (2007). Evaluating resource gain: Understanding and misunderstanding posttraumatic growth. Applied Psychology: An International Review, 56(3), 396-406. htpps://doi.org/10.1111/j.14640597.2007.00299.x

Thoresen, C. E. (1999). Spirituality and Health: Is there a relationship? Journal of Health Psychology, 4(3), 291.

Tjaden, P., \& Thoennes, N. (2006). Extent, nature, and consequences of rape victimization: Findings from the National Violence Against Women Survey (No. NCJ210346). U.S. Department of Justice, National Institute of Justice.

Ullman, S. E. (1999). Social support and recovery from sexual assault: A review. Aggression and Violent Behavior, 4(3), 343-358. doi:http://dx.doi.org/10.1016/S1359-1789(98)00006-8 
U.S. Department of Justice. (October 2018). Criminal Victimization, 2016. https://www.bjs.gov/content/pub/pdf/cv16re.pdf

U.S. Department of Justice. Office of Justice Programs, National Institute of Justice. (2006). Extent, nature, and consequences of rape victimization: Findings from the National Violence Against Women Survey. https://www.ncjrs.gov/pdffiles1/nij/210346.pdf

Van Dyke, C. J., Glenwick, D. S., Cecero, J. J., \& Se-Kang Kim. (2009). The relationship of religious coping and spirituality to adjustment and psychological distress in urban early adolescents. Mental Health, Religion \& Culture, 12(4), 369-383. https://doi-org.ezproxy.andrews.edu/10.1080/13674670902737723

Vanhooren, S., Leijssen, M., \& Dezutter, J. (2018). Coping strategies and posttraumatic growth in prison. Prison Journal, 98(2), 123-142. https://doiorg.ezproxy.andrews.edu/10.1177/0032885517753151

Van Wormer, K., \& Berns, L. (2004). The impact of priest sexual abuse: Female survivors' narratives. Affilia: Journal of Women \& Social Work, 19(1), 53-67. htpps://doi.org/10.1177/0886109903260667

Vilenica, S., Shakespeare-Finch, J., \& Obst, P. (2013). Exploring the process of meaning making in healing and growth after childhood sexual assault: A case study approach. Counselling Psychology Quarterly, 26(1), 39-54. htpps://doi.org/10.1080/09515070.2012.728074 
Walker, D. F., Reid, H. W., O’Neill, T., \& Brown, L. (2009). Changes in personal religion/spirituality during and after childhood abuse: A review and synthesis. Psychological Trauma: Theory, Research, Practice, and Policy, 1(2), 130-145. doi:http://dx.doi.org/10.1037/a0016211

Walker-Williams, H. J., van Eeden, C., \& van der Merwe, K. (2013). Coping behaviour, posttraumatic growth and psychological well-being in women with childhood sexual abuse. Journal of Psychology In Africa (Elliott \& Fitzpatrick, Inc.), 23(2), 259-268.

Webster, J. D., \& Denng, X. C. (2015). Paths from trauma to intrapersonal strength: Worldview, posttraumatic growth, and wisdom. Journal of Loss \& Trauma, 20(3), 253-266. https://doi-org.ezproxy.andrews.edu/10.1080/15325024.2014.932207

Wheaton, B. (2007). The twain meet: Distress, disorder and the continuing conundrum of categories (comment on horwitz). Health: An Interdisciplinary Journal for the Social Study of Health, Illness and Medicine, 11(3), 303-319. doi:http://dx.doi.org.ezproxy.andrews.edu/10.1177/1363459307077545

Wilson, J. Z., Marin, D., Maxwell, K., Cumming, J., Berger, R., Saini, S., Ferguson, W., and Chibnall, J. T. (2016). Association of posttraumatic growth and illness-related burden with psychosocial factors of patient, family, and provider in pediatric cancer survivors. Journal of Traumatic Stress, 29(5), 448-456. https://doiorg.ezproxy.andrews.edu/10.1002/jts. 22123 
Wlodarczyk, A., Basabe, N., Páez, D., Reyes, C., Villagrán, L., Madariaga, C., Palacio, J., \& Martínez, F. (2016). Communal coping and posttraumatic growth in a context of natural disasters in Spain, Chile, and Colombia. Cross-Cultural Research, 50(4), 325-355. htpps://doi.org/10.1177/1069397116663857

Woodward, C., \& Joseph, S. (2003). Positive change processes and post-traumatic growth in people who have experienced childhood abuse: Understanding vehicles of change. Psychology \& Psychotherapy: Theory, Research \& Practice, 76(3), $267-283$.

Wright, M., Crawford, E., \& Sebastian, K. (2007). Positive resolution of childhood sexual abuse experiences: The role of coping, benefit-finding and meaningmaking. Journal of Family Violence, 22(7), 597-608.

htpps://doi.org/10.1007/s10896-007-9111-1

Yilmaz, M., \& Zara, A. (2016). Traumatic loss and posttraumatic growth: The effect of traumatic loss related factors on posttraumatic growth. Anatolian Journal of Psychiatry / Anadolu Psikiyatri Dergisi, 17(1), 5-11. htpps://doi.org/10.5455/apd.188311

Yolom, I. D., \& Lieberman, M. (1991). Bereavement and heightened existential awareness. Psychiatry Interpersonal \& Biological Processes, 54(4), 334-45. htpps://doi.org/10.1521/00332747.1991.11024563

Zinnbauer, B. J., Pargament, K. I., Cole, B., Rye, M. S., Butfer, E. M., Belavich, T. G., Hipp, K. M., Scott, A. B., \& Kadar, J. L. (1997). Religion and spirituality: Unfuzzying the fuzzy. Journal for the Scientific Study of Religion, 36(4), 549564. 
Zinnbauer, B. J., Pargament, K. I., \& Scott, A. B. (1999). The emerging meanings of religiousness and spirituality: Problems and prospects. Journal of Personality, 67(6), 889-919.

Zoellner, T., \& Maercker, A. (2006). Posttraumatic growth in clinical psychology-A critical review and introduction of a two component model. Clinical Psychology Review, 26(5), 626-653. htpps://doi.org/10.1016/j.cpr.2006.01.008 


\section{VITA}

Name: $\quad$ Angela R. St. Hillaire

Education:

Ph.D., Counseling Psychology, 2020

Andrews University, Berrien Springs, MI

M.A. Community Counseling, 2011

Andrews University, Berrien Springs, MI

B.A. Sociology/Social Work, 1983

Atlantic Union College, South Lancaster, MA

\section{Professional Experience:}

2019-Present Registered Psychotherapist (Qualifying), DF Psychological Services, Toronto, ON, Canada

2017-2018 Doctoral Psychology Intern, Centerstone Hospital and Addiction Center, Bradenton, $F L$

2014-2016 Licensed Professional Counselor, Life Coach Psychology

Berrien Springs, $M I$

2014-2016 Group Therapy Facilitator, Well of Grace Ministries

Stevensville, $M I$

2012-2013 Doctoral Practicum in Counseling, Andrews Community Counseling Center, Berrien Spring, MI

2014 Supervisor of M.A. Practicum Students, Andrews Community Counseling Center, Berrien Springs, MI

2010-2011 Master's Internship in Counseling, Southwestern Medical Clinics, Stevensville, $M I$

2010

Master's Practicum in Counseling, Andrews community Counseling Center, Berrien Springs. MI 